\title{
Invariants of boundary link cobordism II. The Blanchfield-Duval form.
}

\author{
Desmond Sheiham
}

\begin{abstract}
We use the Blanchfield-Duval form to define complete invariants for the cobordism group $C_{2 q-1}\left(F_{\mu}\right)$ of $(2 q-1)$-dimensional $\mu$-component boundary links (for $q \geq 2$ ).

The author solved the same problem in earlier work via Seifert forms. Although Seifert forms are convenient in explicit computations, the Blanchfield-Duval form is more intrinsic and appears naturally in homology surgery theory.

The free cover of the complement of a link is constructed by pasting together infinitely many copies of the complement of a $\mu$-component Seifert surface. We prove that the algebraic analogue of this construction, a functor denoted $B$, identifies the author's earlier invariants with those defined here. We show that $B$ is equivalent to a universal localization of categories and describe the structure of the modules sent to zero. Taking coefficients in a semi-simple Artinian ring, we deduce that the Witt group of Seifert forms is isomorphic to the Witt group of Blanchfield-Duval forms.
\end{abstract}

\section{Introduction}

This paper is the second in a series on cobordism (=concordance) groups of a natural class of high-dimensional links. Chapter 1 of the first work [51] discusses background to the problem at greater length but we summarize here some of the key ideas. 


\section{$1.1 \quad$ Background}

A knot is an embedding of spheres ${ }^{1} S^{n} \subset S^{n+2}$. The following are generalizations:

- A $\mu$-component link is an embedding of $\mu$ disjoint spheres

$$
L=\overbrace{S^{n} \sqcup \cdots \sqcup S^{n}}^{\mu} \subset S^{n+2} .
$$

- A boundary link is a link whose components bound disjoint $(n+1)$ manifolds. The union of these $(n+1)$-manifolds is called a Seifert surface.

- An $F_{\mu}$-link is a pair $(L, \theta)$ where $L$ is a link and $\theta$ is a homomorphism from the fundamental group $\pi_{1}(X)$ of the link complement $X=S^{n+2} \backslash L$ onto the free group $F_{\mu}$ on $\mu$ (distinguished) generators such that some meridian of the $i$ th link component is sent to the $i$ th generator.

Not every link is a boundary link; a link $L$ can be refined to an $F_{\mu}$-link $(L, \theta)$ if and only if $L$ is a boundary link.

Let us call a homomorphism $\theta: \pi_{1}(X) \rightarrow F_{\mu}$ permissible if it sends some meridian of the $i$ th link component to the $i$ th generator. There may be many permissible homomorphisms for a given boundary link but if $\theta$ and $\theta^{\prime}$ are permissible then $\theta^{\prime}=\alpha \theta$ where $\alpha$ is some "generator conjugating" automorphism of $F_{\mu}$ (Cappell and Shaneson [6], Ko [27, p660-663]). Homomorphisms $\pi_{1}(X) \rightarrow F_{\mu}$ correspond to homotopy classes of maps from the link complement $X$ to a wedge of $\mu$ circles and the permissible homotopy classes correspond, by the Pontrjagin-Thom construction, to cobordism classes of Seifert surfaces (rel $L$ ).

Every knot is a (1-component) boundary link and admits precisely one permissible homomorphism, namely the abelianization

$$
\theta: \pi_{1}(X) \rightarrow \pi_{1}(X)^{\mathrm{ab}} \cong \mathbb{Z} .
$$

Among the three generalizations above it is the theory of $F_{\mu}$-links which seems to bear the closest resemblance to knot theory.

\footnotetext{
${ }^{1}$ Manifolds are assumed oriented and embeddings are assumed locally flat. One may work in the category of smooth, $P L$ or topological manifolds according to taste, with the understanding that $S^{n}$ is permitted exotic structures if one selects the smooth category.
} 
Although one does not hope for a complete classification of knots or $F_{\mu^{-}}$ links in higher dimensions much is known about their classification up to the equivalence relation known as cobordism (or concordance). Two links $L^{0}$ and $L^{1}$ are called cobordant if there is an embedding

$$
L I=\left(S^{n} \sqcup \cdots \sqcup S^{n}\right) \times[0,1] \subset S^{n+2} \times[0,1]
$$

which joins $L^{0} \subset S^{n+2} \times\{0\}$ to $L^{1} \subset S^{n+2} \times\{1\}$. One requires ${ }^{2}$ that $\left(S^{n} \sqcup \cdots \sqcup S^{n}\right) \times\{i\} \subset S^{n+2} \times\{i\}$ for $i=0$ and $i=1$ but no such requirement is made when $0<i<1$. Boundary links are said to be boundary cobordant if there is a cobordism $L I$ whose components bound disjoint $(n+2)$-manifolds in $S^{n+2} \times[0,1]$. Two $F_{\mu}$-links $\left(L^{0}, \theta^{0}\right)$ and $\left(L^{1}, \theta^{1}\right)$ are called cobordant if there is a pair

$$
\left(L I, \Theta: \pi_{1}\left(S^{n+2} \times[0,1] \backslash L I\right) \rightarrow F_{\mu}\right)
$$

such that the restrictions of $\Theta$ to $\pi_{1}\left(X^{0}\right)$ and $\pi_{1}\left(X^{1}\right)$ coincide with $\theta^{0}$ and $\theta^{1}$ (up to inner automorphism).

The cobordism classes of knots form an abelian group $C_{n}\left(F_{1}\right)$ under (ambient) connected sum but this operation does not extend to links in any obvious way. If one attempts to add links $L^{0}$ and $L^{1}$ there are many inequivalent choices of connecting arc from the $i$ th component of $L^{0}$ to the $i$ th component of $L^{1}$.

However when $n \geq 2$ connected sum $\left[L_{1}, \theta_{1}\right]+\left[L_{2}, \theta_{2}\right]$ of cobordism classes of $F_{\mu}$-links is well-defined; one can remove the ambiguity in the choice of paths by assuming, perhaps after some surgery, that $\theta_{1}$ and $\theta_{2}$ are isomorphisms. The set $C_{n}\left(F_{\mu}\right)$ of cobordism classes of $F_{\mu}$-links is therefore an abelian group.

When $n$ is even, $C_{n}\left(F_{\mu}\right)$ is in fact the trivial group [24, 6, 27, 38]; we sketch a proof in [51, Ch1§4.1]. On the other hand J.Levine obtained a complete system of invariants for odd-dimensional knot cobordism groups $C_{2 q-1}\left(F_{1}\right)$ for $q \geq 2$ [31] and showed that each is isomorphic to a countable direct sum

$$
C_{2 q-1}\left(F_{1}\right) \cong \mathbb{Z}^{\oplus \infty} \oplus\left(\frac{\mathbb{Z}}{2 \mathbb{Z}}\right)^{\oplus \infty} \oplus\left(\frac{\mathbb{Z}}{4 \mathbb{Z}}\right)^{\oplus \infty}
$$

\footnotetext{
${ }^{2} L I$ is also required to meet $S^{n+2} \times\{0\}$ and $S^{n+2} \times\{1\}$ transversely.
} 
The computation of $C_{1}\left(F_{1}\right)$ remains open. In [51] the author obtained a complete system of invariants for odd-dimensional $F_{\mu}$-link cobordism groups $C_{2 q-1}\left(F_{\mu}\right), q \geq 2$ (including some secondary invariants defined only if certain primary invariants vanish) and found that

$$
C_{2 q-1}\left(F_{\mu}\right) \cong \mathbb{Z}^{\oplus \infty} \oplus\left(\frac{\mathbb{Z}}{2 \mathbb{Z}}\right)^{\oplus \infty} \oplus\left(\frac{\mathbb{Z}}{4 \mathbb{Z}}\right)^{\oplus \infty} \oplus\left(\frac{\mathbb{Z}}{8 \mathbb{Z}}\right)^{\oplus \infty}
$$

for all $q \geq 2$ and all $\mu \geq 2$.

Both (1) and (2) were deduced from a purely algebraic reformulation of $F_{\mu}$-link cobordism associated to Seifert surfaces: It was proved by Levine [32] in the knot theory case $\mu=1$ and by Ko [27] and Mio [38] independently in the general case that $C_{2 q-1}\left(F_{\mu}\right)$ is isomorphic to the "Witt group of Seifert forms". In the notation of the present paper, which we explain more carefully in Sections 2.4, 4.1 and 4.3,

$$
C_{2 q-1}\left(F_{\mu}\right) \cong W^{(-1)^{q}}(\mathcal{S} \operatorname{ei}(\mathbb{Z})) \quad(q \geq 3) .
$$

The symbol $\mathcal{S}$ ei $(\mathbb{Z})$ denotes $^{3}$ a category of "Seifert modules" designed to contain the homology modules of Seifert surfaces among the objects (see Notation 4.1). In the case $\mu=1$ an object in $\mathcal{S}$ ei $(\mathbb{Z})$ is a finitely generated free $\mathbb{Z}$-module $V$ together with an endomorphism $V \rightarrow V$ which carries information about how a Seifert surface is embedded. If $\mu>1$ then the definition of Seifert module also includes a direct sum decomposition $V=$ $V_{1} \oplus \cdots \oplus V_{\mu}$ which reflects the connected components of a Seifert surface.

The intersection form in a Seifert surface is an isomorphism $\phi: V \rightarrow V^{*}$ in $\mathcal{S}$ ei $(\mathbb{Z})$ which satisfies $\phi^{*}=(-1)^{q} \phi$. Such $(-1)^{q}$-hermitian forms are the generators of the Witt group $W^{(-1)^{q}}(\mathcal{S}$ ei $(\mathbb{Z}))$. The relations say that certain "metabolic forms" are identified with zero; see Definitions 2.22 and 2.23 below.

Although Seifert surface methods are convenient in explicit computations, it is preferable to define $F_{\mu}$-link invariants without making a choice of Seifert surface. In the present paper we focus instead on the covering space $\bar{X} \rightarrow X$ of a link complement determined by the homomorphism $\theta: \pi_{1}(X) \rightarrow F_{\mu}$. This approach sits more naturally in homology surgery theory and is more amenable to generalization from boundary links to arbitrary links or other manifold embeddings.

We take as starting point the identification

$$
C_{2 q-1}\left(F_{\mu}\right) \cong W^{(-1)^{q+1}}(\mathcal{F} \operatorname{lk}(\mathbb{Z})) \quad(q \geq 3)
$$

\footnotetext{
${ }^{3}$ The category $\mathcal{S}$ ei $(\mathbb{Z})$ was denoted $\left(P_{\mu}-\mathbb{Z}\right)$-Proj in [51].
} 
where $\mathcal{F l k}(\mathbb{Z})$ is a category designed to contain homology modules of the cover $\bar{X}$ (see Definition 2.1 and Notation 2.2). The objects in $\mathcal{F} \operatorname{lk}(\mathbb{Z})$ are certain modules over the group ring $\mathbb{Z}\left[F_{\mu}\right]$ of the free group; they are called $F_{\mu}$-link modules in the present paper although they are more commonly known as link modules.

The $F_{\mu}$-equivariant Poincaré duality in $\bar{X}$ leads to a $(-1)^{q+1}$-hermitian form $\phi$ in the category $\mathcal{F} \operatorname{lk}(\mathbb{Z})$. This is the Blanchfield-Duval form of the title, originally introduced by Blanchfield [3] in the knot theory case $\mu=1$. The identity (4) was proved by Kearton for $\mu=1[23,22]$ and by Duval [14] for $\mu \geq 2$. Cappell and Shaneson earlier identified the cobordism group $C_{n}\left(F_{\mu}\right)$ with a $\Gamma$-group, an obstruction group in their homology surgery theory $[5,6]$. The identification of this $\Gamma$-group with the Witt group $W^{(-1)^{q+1}}(\mathcal{F} \operatorname{lk}(\mathbb{Z}))$ was due to Pardon [39, 40], Ranicki $[42, \S 7.9]$ and Smith [52] for $\mu=1$ and to Duval [14] for $\mu \geq 2$. More general results of Vogel $[54,55]$ on homology surgery and universal localization are stated elsewhere in this volume $[45, \S 1.4]$. An outline of their application to $C_{n}\left(F_{\mu}\right)$ is given in $[51, \mathrm{Ch} 1, \S 4.4,5.3]$.

\subsection{Overview}

Universal localization plays two roles in this paper. Firstly the "augmentation localization" of the group ring $\mathbb{Z}\left[F_{\mu}\right]$ of the free group appears in the definition of the Blanchfield-Duval form, our main object of study. Secondly, we prove that the category $\mathcal{F l k}(\mathbb{Z})$ of $F_{\mu}$-link modules is (equivalent to) a universal localization of the category $\mathcal{S}$ ei $(\mathbb{Z})$ of Seifert modules.

Our first aim is to use (4) to distinguish the elements of $C_{2 q-1}\left(F_{\mu}\right)$. We define complete invariants (and secondary invariants if certain primary invariants vanish) by analyzing the Witt groups $W^{(-1)^{q+1}}(\mathcal{F l k}(\mathbb{Q}))$. We proceed in three steps, explained in more detail in Section 3, which run parallel to steps 2, 3 and 4 in chapter 2 of [51]:

1. Obtain a direct sum decomposition of $W^{(-1)^{q+1}}(\mathcal{F} \operatorname{lk}(\mathbb{Q}))$ by "devissage". One must prove that $\mathcal{F} \operatorname{lk}(\mathbb{Q})$ is an abelian category in which each module has a finite composition series.

2. Use hermitian Morita equivalence to show that each summand of the group $W^{(-1)^{q+1}}(\mathcal{F} \operatorname{lk}(\mathbb{Q}))$ is isomorphic to some group $W^{1}(E)$ where $E$ is a division ring of finite dimension over $\mathbb{Q}$.

3. Recall from the literature invariants of each $W^{1}(E)$. 
In the knot theory case $\mu=1$ there is one summand of $W^{(-1)^{q+1}}(\mathcal{F} \operatorname{lk}(\mathbb{Q}))$ for each maximal ideal $(p) \in \mathbb{Q}\left[z, z^{-1}\right]$ which is invariant under the involution $z \mapsto z^{-1}$. The generator $p$ is often called an Alexander polynomial. The division ring $E$ coincides with the quotient field $\mathbb{Q}\left[z, z^{-1}\right] /(p)$ and $W^{1}(E)$ is the Witt group of hermitian forms over $E$ (compare Milnor [37]).

The following theorem and corollary are restated and proved in Section 3; see Theorem 3.2 and Corollary 3.3.

Theorem 1.1. The invariants (and secondary invariants) defined in Section 3 are sufficient to distinguish the elements of the Witt groups $W^{ \pm}(\mathcal{F} \operatorname{lk}(\mathbb{Q}))$ of Blanchfield-Duval forms with coefficients in $\mathbb{Q}$.

Corollary 1.2. Let $q>1$ and suppose $\phi^{0}$ and $\phi^{1}$ are the Blanchfield-Duval forms for the $(2 q-1)$-dimensional $F_{\mu}$-links $\left(L^{0}, \theta^{0}\right)$ and $\left(L^{1}, \theta^{1}\right)$ respectively. These two $F_{\mu}$-links are cobordant if and only if all the invariants (and possible secondary invariants) of

$$
\left[\mathbb{Q} \otimes_{\mathbb{Z}}\left(\phi^{0} \oplus-\phi^{1}\right)\right] \in W^{(-1)^{q+1}}(\mathcal{F} \operatorname{lk}(\mathbb{Q}))
$$

defined in Section 3 are trivial.

Corollary 1.2 follows from (4) and the fact that the canonical map

$$
W^{(-1)^{q+1}}(\mathcal{F} \operatorname{lk}(\mathbb{Z})) \rightarrow W^{(-1)^{q+1}}(\mathcal{F} \operatorname{lk}(\mathbb{Q}))
$$

is an injection, which we deduce from Theorem 1.3 at the end of Section 3.1. Corollary 1.2 is also a consequence of Theorem 1.4 and Theorem B of [51].

Our second aim is to understand the algebraic relationship between the Seifert forms and the Blanchfield-Duval form of an $F_{\mu}$-link and prove that the cobordism invariants defined in [51] using Seifert forms are equivalent to those defined in Section 3 via the Blanchfield-Duval form. Example 4.3 gives a sample calculation of the Seifert form invariants in [51].

In the knot theory case $\mu=1$, the relationship between Seifert and Blanchfield forms has been investigated extensively by Kearton [23], Levine [33, $\S 14]$, Farber [15, §7.1] and Ranicki ([43, ch32],[44]). For $\mu \geq 1$ K.H.Ko [28] used geometric arguments to obtain a formula for Cappell and Shaneson's homology surgery obstruction in terms of the Seifert form. A formula for the Blanchfield-Duval form in terms of the Seifert form, again based on geometric arguments, can also be found in Cochran and Orr [7, Thm4.2] in the slightly more general context of "homology boundary links".

M.Farber related Seifert and Blanchfield-Duval forms of $F_{\mu}$-links in a purely algebraic way $[16,17]$. Although the present paper is logically independent of his work, we take up a number of his ideas in Sections 4 and 5, 
providing a systematic treatment in the language of hermitian categories. Whereas Farber takes coefficients in a field or in $\mathbb{Z}$, in these sections we allow the coefficients to lie in an arbitrary associative ring $A$.

The first step is to show that an $F_{\mu}$-link module admits a canonical Seifert module structure (cf $[16, \mathrm{p} 193])$. An $F_{\mu}$-link module $M \in \mathcal{F} \operatorname{lk}(A)$ is not in general finitely generated (or projective) as an $A$-module so we introduce a larger category $\mathcal{S} \mathrm{ei}_{\infty}(A)$ which contains $\mathcal{S} \mathrm{ei}(A)$ as a full subcategory (see Notation 4.1). We obtain a "forgetful" functor

$$
U: \mathcal{F} \operatorname{lk}(A) \rightarrow \mathcal{S} \mathrm{ei}_{\infty}(A)
$$

For example, in the case $\mu=1$ of knot theory, an object in $\mathcal{F l k}(A)$ is a module $M$ over the ring $A\left[z, z^{-1}\right]$ of Laurent polynomials with a presentation

$$
0 \rightarrow\left(A\left[z, z^{-1}\right]\right)^{m} \stackrel{\sigma}{\rightarrow}\left(A\left[z, z^{-1}\right]\right)^{m} \rightarrow M \rightarrow 0
$$

such that $1-z: M \rightarrow M$ is an isomorphism. The Seifert module $U(M)$ is the $A$-module $M$ together with the endomorphism $(1-z)^{-1}$.

If $A=k$ is a field, Farber defined, for each $M \in \mathcal{F} \operatorname{lk}(k)$, the "minimal lattice" [16, p194-199] of $M$, a Seifert submodule of $U(M)$ which is of finite $k$-dimension. We prefer to work directly with $U(M)$ which is defined regardless of the coefficients and avoids technicalities of Farber's definition. His minimal lattice becomes isomorphic to $U(M)$ after one performs a universal localization of categories which we describe a few paragraphs below.

Given a Seifert surface for an $F_{\mu}$-link one can construct the free cover by cutting the link complement along the Seifert surface and gluing together infinitely many copies of the resulting manifold in the pattern of the Cayley graph of $F_{\mu}$. Figure 1 illustrates the geometric construction in the case of a 2-component link.

The algebraic analogue of this geometric construction is a functor

$$
B: \mathcal{S e i}(A) \rightarrow \mathcal{F} \operatorname{lk}(A)
$$

from Seifert modules to $F_{\mu}$-link modules (see Definition 5.1). Since $U$ takes values in the larger category $\mathcal{S e i}_{\infty}(A)$ we expand the domain of $B$ to $\mathcal{S} \mathrm{ei}_{\infty}(A)$, by necessity replacing $\mathcal{F} \operatorname{lk}(A)$ by a larger category $\mathcal{F} \mathrm{k}_{\infty}(A)$. This process of enlargement stops here for there are functors

$$
\begin{aligned}
& U: \mathcal{F} \operatorname{lk}_{\infty}(A) \rightarrow \mathcal{S} \operatorname{ei}_{\infty}(A) \\
& B: \mathcal{S} \mathrm{ei}_{\infty}(A) \rightarrow \mathcal{F} \mathrm{lk}_{\infty}(A) .
\end{aligned}
$$




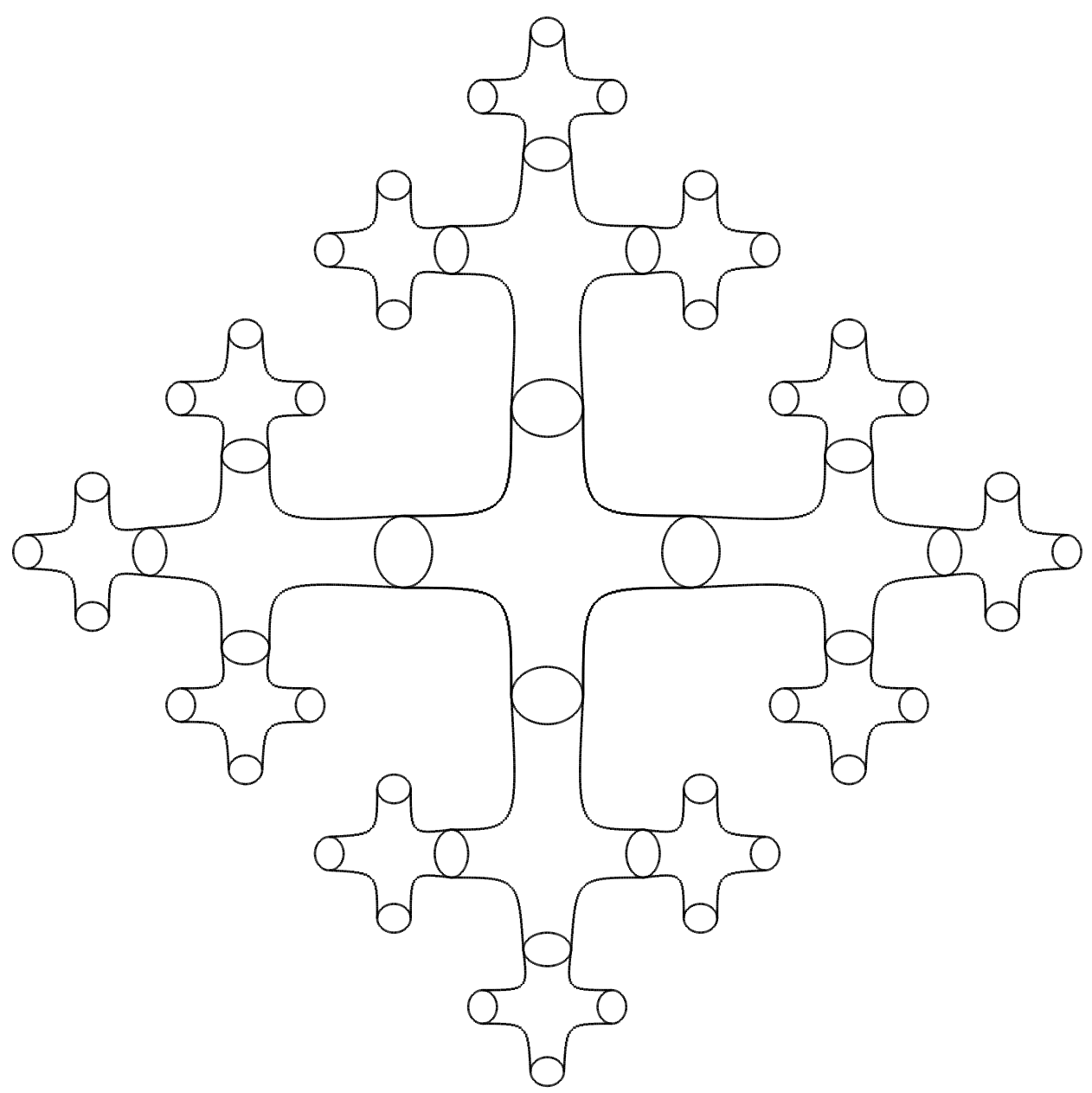

Figure 1

We show in Section 5.2 that $B$ is left adjoint to $U$. Roughly speaking, this means that $B(V)$ is the "free" $F_{\mu}$-link module generated by the Seifert module $V$ (with respect to the functor $U$ ). In other words, $B$ is universal (up to equivalence) among functors from Seifert modules to $F_{\mu}$-link modules.

Returning our attention to the subcategories $\mathcal{S} \operatorname{ei}(A)$ and $\mathcal{F} \operatorname{lk}(A)$ whose definitions involve a "finitely generated projective" condition we show that $B$ is compatible with the notions of duality in $\mathcal{S e i}(A)$ and $\mathcal{F} \operatorname{lk}(A)$, extending 
$B$ to a "duality-preserving functor" between "hermitian categories"

$$
(B, \Phi,-1): \mathcal{S} \operatorname{ei}(A) \rightarrow \mathcal{F} \operatorname{lk}(A) .
$$

(see definitions 2.13 and 2.24 and proposition 5.4). The following theorem concerns the induced homomorphism of Witt groups:

$$
B: W^{ \pm}(\mathcal{S} \operatorname{ei}(A)) \rightarrow W^{\mp}(\mathcal{F} \operatorname{lk}(A)) .
$$

Recall that by Wedderburn's Theorem, a ring $A$ is semi-simple and Artinian if and only if it is a product of matrix rings over division rings.

Theorem 1.3. If $A$ is a semi-simple Artinian ring then (6) is an isomorphism.

The map (6) will be considered for more general rings $A$ in subsequent work (joint with A.Ranicki) [46]. It follows from the isomorphisms (3) and (4) above that (6) is an isomorphism when $A=\mathbb{Z}$.

Theorem 1.4. The duality-preserving functor $(B, \Phi,-1)$ identifies the Seifert form invariants of [51] with the Blanchfield-Duval form invariants of Section 3.

Theorem 6.5 below is a more precise statement of Theorem 1.4. The invariants of [51] are outlined in Section 4.3. Theorem 1.3 is proved in two stages. The first stage is to establish that, for any ring $A$, there is an equivalence between $(B, \Phi,-1)$ and a certain universal localization of hermitian categories. Taking up Farber's terminology we call a Seifert module $V \in \mathcal{S e i}_{\infty}(A)$ primitive if $B(V) \cong 0$. We denote by $\mathcal{P r i m}_{\infty}(A)$ the category of primitive modules. One may write

$$
\operatorname{Prim}_{\infty}(A)=\operatorname{Ker}\left(B: \mathcal{S} \mathrm{ei}_{\infty}(A) \rightarrow \mathcal{F} \mathrm{lk}_{\infty}(A)\right) .
$$

The category quotient

$$
F: \mathcal{S} \operatorname{ei}_{\infty}(A) \rightarrow \mathcal{S} \operatorname{ei}_{\infty}(A) / \mathcal{P} \operatorname{rim}_{\infty}(A)
$$

is universal among functors which make invertible morphisms whose kernel and cokernel are primitive. In particular, primitive modules in $\mathcal{S} \mathrm{ei}_{\infty}(A)$ are made isomorphic to 0 in $\mathcal{S e i}_{\infty}(A) / \mathcal{P} \operatorname{rim}_{\infty}(A)$.

Since $B: \mathcal{S e i}_{\infty}(A) \rightarrow \mathcal{F} \mathrm{lk}_{\infty}(A)$ is left adjoint to $U$ it follows that $B$ exhibits the same universal property as $F$ although only "up to natural isomorphism" (Proposition 5.14). We conclude that $B$ is equivalent to $F$ 
and, with a little extra work, establish that $(B, \Phi,-1): \mathcal{S} \operatorname{ei}(A) \rightarrow \mathcal{F} \operatorname{lk}(A)$ is equivalent to a universal localization of hermitian categories (Theorem 5.17 and Proposition 5.22).

In the knot theory case $\mu=1$ the category $\mathcal{S e i}_{\infty}(A)$ coincides with the category of (left) modules over the polynomial ring $A[s]$ in a central indeterminate $s$. Setting $t=s(1-s)$, the functor (7) is the central localization $A\left[s, t^{-1}\right] \otimes_{A[s]}$ - from the category of $A[s]$-modules to the category of $A\left[s, t^{-1}\right]$-modules; see Farber [15, Thm2.6] and Ranicki [44].

Pere Ara recently gave an independent proof [2, Thm 6.2] using Farber's minimal lattice that if $A=k$ is a field then $\mathcal{F l k}(k)$ is equivalent to a localization of $\mathcal{S e i}(k)$ by a category of primitive modules (for all $\mu \geq 1)^{4}$.

The second stage in the proof of Theorem 1.3 involves the analysis of primitive modules. A Seifert module $V$ is called "trivially primitive" if the endomorphism with which it is endowed is either zero or the identity. In Proposition 5.28 we show that every primitive module $V \in \mathcal{P r i m}_{\infty}(A)$ is composed of (possibly infinitely many) trivially primitive modules. Restricting attention to $\mathcal{S e i}(A)$ we show that if $A$ is semi-simple and Artinian then every primitive module is composed of finitely many trivially primitive modules in $\mathcal{S}$ ei $(A)$ (cf Farber $[17, \S 3, \S 7.10]$ ). The proof of Theorem 1.3 is completed in Section 6.1 by establishing that the Witt group of the subcategory $\operatorname{Prim}(A) \subset \mathcal{S}$ ei $(A)$ of primitive modules is trivial. See Proposition 6.3 and part 2. of Lemma 6.4.

The definitions of Blanchfield-Duval form invariants in Section 3 parallel the author's Seifert form invariants in [51, Ch2]. The three steps outlined above to analyze $W^{ \pm}(\mathcal{F} \operatorname{lk}(\mathbb{Q}))$ were applied to $W^{ \pm}(\mathcal{S}$ ei $(\mathbb{Q}))$ in [51]. Theorem 1.4 is proved in Section 6.2 by checking that the duality-preserving functor $(B, \Phi,-1)$ respects each of these three steps.

Let us summarize the contents of this paper. Section 2 discusses $F_{\mu^{-}}$ link modules over an arbitrary ring $A$ and uses universal localization (cf Vogel $[54,55]$ and Duval [14]) to describe hermitian structure in the category $\mathcal{F l k}(A)$. We define the Witt groups $W^{ \pm}(\mathcal{F l k}(A))$ of Blanchfield-Duval forms.

In Section 3 we set $A=\mathbb{Q}$ and define invariants of $W^{ \pm}(\mathcal{F} \operatorname{lk}(\mathbb{Q}))$, obtain-

\footnotetext{
${ }^{4}$ His context differs slightly in that the free algebra $k\langle X\rangle$ on a set $X=\left\{x_{1}, \cdots, x_{\mu}\right\}$ takes the place of the group ring $k\left[F_{\mu}\right]$ in the present paper; the category denoted $\mathcal{Z}$ in [2] plays the role of $\mathcal{F} \operatorname{lk}(k)$. Consequently, there is only one kind of "trivially primitive" module (denoted $M_{0}$ in [2]) as compared with the two kinds in [17] and Section 5.5 below. Ara also related the modules in $\mathcal{Z}$ to modules over the Leavitt algebra $L$. By definition, $L$ is the universal localization of $k\langle X\rangle$ which makes invertible the map $\left(x_{1} \cdots x_{\mu}\right): k\langle X\rangle^{\mu} \rightarrow$ $k\langle X\rangle$ (compare the Sato condition, Lemma 2.3 below). The category $\mathcal{Z}$ turns out to be equivalent to the category of finitely presented $L$-modules of finite length.
} 
ing intrinsic cobordism invariants for $F_{\mu}$-links. We discuss each of the steps 1-3. listed above, reformulating and proving Theorem 1.1 and Corollary 1.2.

In Section 4 we define Seifert modules and Seifert forms with coefficients in an arbitrary ring $A$. We treat a worked example of the invariants defined in [51] and we define a forgetful functor $U: \mathcal{F} \mathrm{k}_{\infty}(A) \rightarrow \mathcal{S} \mathrm{ei}_{\infty}(A)$.

Section 5 begins to study the functor $B: \mathcal{S e i}_{\infty}(A) \rightarrow \mathcal{F} \mathrm{lk}_{\infty}(A)$ from Seifert modules to $F_{\mu}$-link modules. We prove that $B$ is left adjoint to $U: \mathcal{F} \mathrm{lk}_{\infty}(A) \rightarrow \mathcal{S} \mathrm{ei}_{\infty}(A)$ and show that $B$ factors through a category equivalence $\mathcal{S} \operatorname{ei}_{\infty}(A) / \mathcal{P} \operatorname{rim}_{\infty}(A) \rightarrow \mathcal{F} \mathrm{k}_{\infty}(A)$. We describe the structure of the primitive modules - those which are sent to zero by $B$ - and outline a construction of the localization $\mathcal{S e i}_{\infty}(A) \rightarrow \mathcal{S} \mathrm{ei}_{\infty}(A) / \mathcal{P} \operatorname{rim}_{\infty}(A)$. We construct a duality-preserving functor $(B, \Phi,-1): \mathcal{S} \operatorname{ei}(A) \rightarrow \mathcal{F} \operatorname{lk}(A)$ which is natural in $A$ and factors through an equivalence $\mathcal{S} \operatorname{ei}(A) / \mathcal{P} \operatorname{rim}_{\infty}(A) \rightarrow$ $\mathcal{F} \operatorname{lk}(A)$ of hermitian categories. If $A$ is a semi-simple Artinian ring we give a simplified description of the primitive modules and the universal localization of hermitian categories.

Section 6 contains a proof of Theorem 1.3 and a reformulation and proof of Theorem 1.4.

Acknowledgments: The invariants in Section 3 came into being during my thesis work at the University of Edinburgh under the guidance of Andrew Ranicki. I am also indebted to Andrew for several more recent conversations and e-mails and for encouragement to complete this paper. I am grateful to John Baez and James Dolan for helpful discussions in category theory, and to Pere Ara and to the referee for their comments and corrections.

I thank the London Mathematical Society for financial support to attend the ICMS workshop on "Noncommutative Localization in Algebra and Topology" in April 2002 and the Edinburgh Mathematical Society who financed my visit to Edinburgh in August 2002.

\section{The Blanchfield-Duval form}

\section{$2.1 \quad F_{\mu}$-link Modules}

Let $A$ be an associative ring with 1 . Modules will be left modules except where otherwise stated. Let $A\left[F_{\mu}\right]$ denote the group ring of the free group $F_{\mu}$; an element of $A\left[F_{\mu}\right]$ is a formal sum of elements of $F_{\mu}$, with coefficients in $A$. Note that elements of the group $F_{\mu}$ commute with elements of $A$ and $A\left[F_{\mu}\right] \cong A \otimes_{\mathbb{Z}} \mathbb{Z}\left[F_{\mu}\right]$. 
The symbols $\epsilon$ and $j$ will be used for three slightly distinct purposes but the meaning will be clear from the context. Firstly, $j$ denotes the inclusion of $A$ in $A\left[F_{\mu}\right]$. Secondly $j$ denotes the functor $V \mapsto A\left[F_{\mu}\right] \otimes_{A} V$ from the category of $A$-modules to the category of $A\left[F_{\mu}\right]$-modules. For brevity we write $V\left[F_{\mu}\right]$ in place of $A\left[F_{\mu}\right] \otimes_{A} V$. Thirdly, we use $j$ to denote the inclusion of a module $V$ in $V\left[F_{\mu}\right]$ given by

$$
V \cong A \otimes_{A} V \stackrel{j \otimes 1}{\longrightarrow} A\left[F_{\mu}\right] \otimes_{A} V=V\left[F_{\mu}\right] .
$$

In the opposite direction $\epsilon: A\left[F_{\mu}\right] \rightarrow A$ denotes the ring morphism which sends every element of $F_{\mu}$ to $1 \in A$ and is the identity on $A$. We also write $\epsilon$ for the functor $A \otimes_{A\left[F_{\mu}\right]}$ - from the category of $A\left[F_{\mu}\right]$-modules to the category of $A$-modules. Thirdly, $\epsilon: V\left[F_{\mu}\right] \rightarrow V$ denotes the morphism

$$
V\left[F_{\mu}\right]=A\left[F_{\mu}\right] \otimes_{A} V \stackrel{\epsilon \otimes 1}{\longrightarrow} A \otimes_{A} V \cong V .
$$

Note that the composite $\epsilon j$ of ring morphisms is the identity $\operatorname{id}_{A}$ and the composite $\epsilon j$ of module morphisms is the identity on $V$. The composite $\epsilon j$ of functors is naturally isomorphic to the identity functor on $A$-modules and we sometimes suppress the natural isomorphism identifying $A \otimes_{A\left[F_{\mu}\right]}$ $\left(A\left[F_{\mu}\right] \otimes_{A} V\right)$ with $V$.

Definition 2.1. An $F_{\mu}$-link module is an $A\left[F_{\mu}\right]$-module $M$ which lies in an exact sequence:

$$
0 \rightarrow V\left[F_{\mu}\right] \stackrel{\sigma}{\rightarrow} V\left[F_{\mu}\right] \rightarrow M \rightarrow 0
$$

such that $V$ is an $A$-module and $\epsilon(\sigma): V \rightarrow V$ is an isomorphism.

As we remarked in the introduction, the examples of $F_{\mu}$-link modules in the literature are more often called "link modules". Note that if $V$ is a finitely generated $A$-module then the $F_{\mu}$-link module $M$ is finitely generated as an $A\left[F_{\mu}\right]$-module but usually not as an $A$-module (see Lemma 2.3 below).

It will be helpful to make the following observation about the definition of $F_{\mu}$-link modules: The condition that $\epsilon(\sigma)$ is an isomorphism implies that $\sigma$ is an injection (see Lemma 2.8 below).

Notation 2.2. Let $\mathcal{F} \mathrm{lk}_{\infty}(A)$ denote the category of $F_{\mu}$-link modules and $A\left[F_{\mu}\right]$-module homomorphisms. Thus $\mathcal{F} \mathrm{lk}_{\infty}(A)$ is a full subcategory of the category of $A\left[F_{\mu}\right]$-modules.

Let $\mathcal{F} \operatorname{lk}(A) \subset \mathcal{F} \mathrm{lk}_{\infty}(A)$ denote the category of modules with a presentation (8) such that $V$ is a finitely generated projective $A$-module and $\epsilon(\sigma)$ is an isomorphism. The morphisms in $\mathcal{F} \operatorname{lk}(A)$ are, as usual, the $A\left[F_{\mu}\right]$-module morphisms so $\mathcal{F} \operatorname{lk}(A)$ is a full subcategory of $\mathcal{F} \mathrm{lk}_{\infty}(A)$. 
We show in Lemma 2.9 below that $\mathcal{F} \mathrm{lk}_{\infty}\left({ }_{-}\right)$and $\mathcal{F} \operatorname{lk}\left({ }_{-}\right)$are functorial in $A$. The following lemma gives an alternative characterization of $F_{\mu}$-link modules. Let $z_{1}, \cdots, z_{\mu}$ denote generators for $F_{\mu}$.

Lemma 2.3. (Sato [47]) Suppose $M$ is an $A\left[F_{\mu}\right]$-module which has a presentation

$$
0 \rightarrow V\left[F_{\mu}\right] \stackrel{\sigma}{\rightarrow} V^{\prime}\left[F_{\mu}\right] \rightarrow M \rightarrow 0 .
$$

for some A-modules $V$ and $V^{\prime}$. The augmentation $\epsilon(\sigma): V \rightarrow V^{\prime}$ is an isomorphism if and only if the A-module homomorphism

$$
\begin{aligned}
\gamma: M^{\oplus \mu} & \rightarrow M \\
\left(m_{1}, \cdots, m_{\mu}\right) & \mapsto \sum_{i=1}^{\mu}\left(1-z_{i}\right) m_{i}
\end{aligned}
$$

is an isomorphism.

Proof. There is an exact sequence

$$
0 \rightarrow\left(A\left[F_{\mu}\right]\right)^{\oplus \mu} \stackrel{\gamma}{\rightarrow} A\left[F_{\mu}\right] \stackrel{\epsilon}{\rightarrow} A \rightarrow 0
$$

where $\gamma\left(l_{1}, \cdots, l_{\mu}\right)=\sum_{i=1}^{\mu}\left(1-z_{i}\right) l_{i}$ for all $l_{1}, \cdots, l_{\mu} \in A\left[F_{\mu}\right]$. Now (11) is split (by $j$ ) when regarded as a sequence of right $A$-modules so the functors $\otimes_{A} V$ and $\_\otimes_{A} V^{\prime}$ lead to a commutative diagram

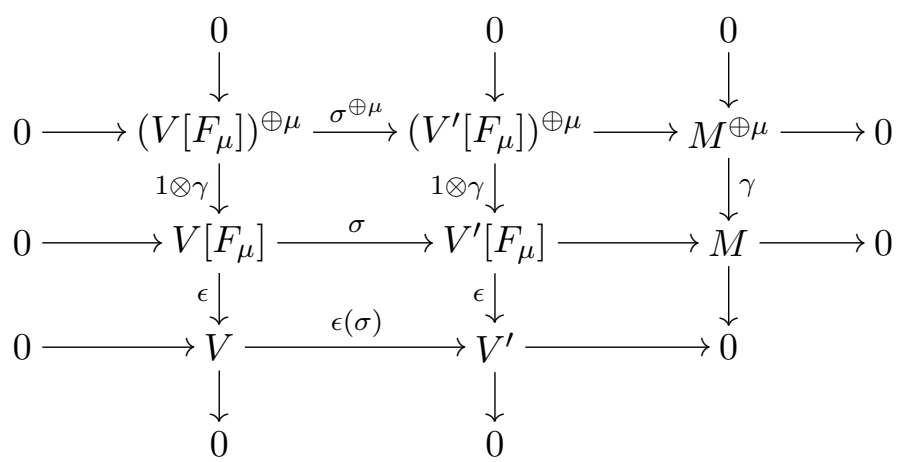

in which the first two rows and the first two columns are exact. A standard diagram chase (e.g. [36, p49]) shows that the third row is exact if and only if the third column is exact and the Lemma follows.

We discuss next completions of $A\left[F_{\mu}\right]$-modules, which we shall need both to refine Definition 2.1 (in Lemma 2.8) and later to study the universal augmentation localization of $A\left[F_{\mu}\right]$ (see Lemma 2.15). 
Let $I=\operatorname{Ker}\left(\epsilon: A\left[F_{\mu}\right] \rightarrow A\right)$. If $N$ is an $A\left[F_{\mu}\right]$-module one defines

$$
\widehat{N}=\lim _{n} \frac{N}{I^{n} N} \text {. }
$$

An $A\left[F_{\mu}\right]$-module morphism $N \rightarrow N^{\prime}$ maps $I^{n} N$ to $I^{n} N^{\prime}$ for each $n$ and therefore induces a homomorphism $\widehat{N} \rightarrow \widehat{N^{\prime}}$.

Caveat 2.4. The natural isomorphisms $\left(A\left[F_{\mu}\right] / I^{n}\right) \otimes_{A\left[F_{\mu}\right]} N \rightarrow N /\left(I^{n} N\right)$ induce a map $\widehat{A\left[F_{\mu}\right]} \otimes N \rightarrow \widehat{N}$ but the latter is not in general an isomorphism.

In the examples with which we are most concerned, $N=V\left[F_{\mu}\right]=$ $A\left[F_{\mu}\right] \otimes_{A} V$ for some $A$-module $V$. One can describe $\widehat{V\left[F_{\mu}\right]}$ as a module of power series as follows. Let $X=\left\{x_{1}, \cdots, x_{\mu}\right\}$ and let $\mathbb{Z}\langle X\rangle$ denote the free ring generated by $X$ (in other words the ring of "polynomials" in noncommuting indeterminates $\left.x_{1}, \cdots, x_{\mu}\right)$. Let $A\langle X\rangle=A \otimes_{\mathbb{Z}} \mathbb{Z}\langle X\rangle$ so that the elements of $A$ are formal sums of words in the alphabet $X$ with coefficients in $A$. Let $V\langle X\rangle$ be the $A\langle X\rangle$-module $A\langle X\rangle \otimes_{A} V$ and denote by $X^{n} V\langle X\rangle \subset V\langle X\rangle$ the submodule whose elements are formal sums of words of length at least $n$ with coefficients in $V$. We may now define the $X$-adic completion

$$
V\langle\langle X\rangle\rangle=\lim _{n} \frac{V\langle X\rangle}{X^{n} V\langle X\rangle} .
$$

in which an element is a formal power series in non-commuting indeterminates $x_{1}, \cdots, x_{\mu}$ with coefficients in $V$.

Lemma 2.5. There is a natural isomorphism $V\langle\langle X\rangle\rangle \cong \widehat{V\left[F_{\mu}\right]}$.

Proof. The required isomorphism is induced by the isomorphisms

$$
\begin{aligned}
\frac{V\left[F_{\mu}\right]}{I^{n} V\left[F_{\mu}\right]} & \cong \frac{V\langle X\rangle}{X^{n} V\langle X\rangle} \\
z_{i} & \mapsto 1+x_{i} \\
z_{i}^{-1} & \mapsto 1-x_{i}+x_{i}^{2} \cdots+(-1)^{n-1} x_{i}^{n-1}
\end{aligned}
$$

Lemma 2.6. If $V$ is an $A$-module, the canonical map $V\left[F_{\mu}\right] \rightarrow \widehat{V\left[F_{\mu}\right]}$ is an injection.

Proof. The argument of Fox $\left[19\right.$, Corollary 4.4] implies that $\bigcap_{n=0}^{\infty} I^{n} V\left[F_{\mu}\right]=0$ and the Lemma follows. 
Lemma 2.7. If $V$ is an $A$-module and $\tau: V\left[F_{\mu}\right] \rightarrow V\left[F_{\mu}\right]$ is an $A\left[F_{\mu}\right]$ module morphism such that $\epsilon(\tau)=0: V \rightarrow V$ then $\tau\left(V\left[F_{\mu}\right]\right) \subset I V\left[F_{\mu}\right]$ and the map $1+\tau: \widehat{V\left[F_{\mu}\right]} \rightarrow \widehat{V\left[F_{\mu}\right]}$ is invertible.

Proof. The commutative diagram

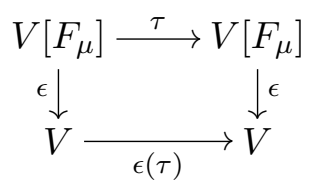

implies that if $\epsilon(\tau)=0$ then

$$
\tau\left(V\left[F_{\mu}\right]\right) \subseteq \operatorname{Ker}\left(\epsilon: V\left[F_{\mu}\right] \rightarrow V\right)=\operatorname{Ker}\left(\epsilon \otimes 1: A\left[F_{\mu}\right] \otimes_{A} V \rightarrow A \otimes_{A} V\right)
$$

Since the surjection $\epsilon: A\left[F_{\mu}\right] \rightarrow A$ is split by $j$ we obtain

$$
\tau\left(V\left[F_{\mu}\right]\right) \subseteq I \otimes_{A} V=I\left(A\left[F_{\mu}\right] \otimes_{A} V\right)=I V\left[F_{\mu}\right] .
$$

By induction, $\tau^{n}\left(V\left[F_{\mu}\right]\right) \subset I^{n} V\left[F_{\mu}\right]$ for all $n$ so $1+\tau: \widehat{V\left[F_{\mu}\right]} \rightarrow \widehat{V\left[F_{\mu}\right]}$ has inverse

$$
(1+\tau)^{-1}=1-\tau+\tau^{2}-\tau^{3}+\cdots .
$$

Lemma 2.8. If $V$ and $V^{\prime}$ are $A$-modules and $\sigma: V\left[F_{\mu}\right] \rightarrow V^{\prime}\left[F_{\mu}\right]$ is an $A\left[F_{\mu}\right]$-module homomorphism such that $\epsilon(\sigma): V \rightarrow V^{\prime}$ is an isomorphism then $\sigma$ is an injection and the induced map $\sigma: \widehat{V\left[F_{\mu}\right]} \rightarrow \widehat{V^{\prime}\left[F_{\mu}\right]}$ is an isomorphism.

Proof. Let $\sigma^{\prime}=(j \epsilon(\sigma))^{-1} \sigma: V\left[F_{\mu}\right] \rightarrow V\left[F_{\mu}\right]$. Now $\epsilon\left(\sigma^{\prime}\right)=1_{V\left[F_{\mu}\right]}$ so we may write $\sigma^{\prime}=1+\tau$ where $\epsilon(\tau)=0$. Now $\sigma^{\prime}: \widehat{V\left[F_{\mu}\right]} \rightarrow \widehat{V\left[F_{\mu}\right]}$ is an isomorphism by Lemma 2.7 so $\sigma: \widehat{V\left[F_{\mu}\right]} \rightarrow \widehat{V^{\prime}\left[F_{\mu}\right]}$ is an isomorphism. The commutative diagram

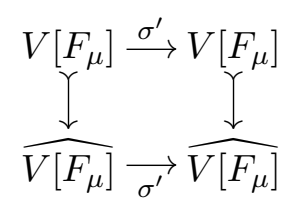

implies that $\sigma^{\prime}: V\left[F_{\mu}\right] \rightarrow V\left[F_{\mu}\right]$ and $\sigma: V\left[F_{\mu}\right] \rightarrow V^{\prime}\left[F_{\mu}\right]$ are injections.

Lemma 2.9. A homomorphism $A \rightarrow A^{\prime}$ of rings induces functors

$$
\begin{aligned}
& A^{\prime}\left[F_{\mu}\right] \otimes_{A\left[F_{\mu}\right]}:: \mathcal{F} \operatorname{lk}_{\infty}(A) \rightarrow \mathcal{F} \operatorname{lk}_{\infty}\left(A^{\prime}\right) \quad \text { and } \\
& A^{\prime}\left[F_{\mu}\right] \otimes_{A\left[F_{\mu}\right]}: \mathcal{F} \operatorname{lk}(A) \rightarrow \mathcal{F} \operatorname{lk}\left(A^{\prime}\right)
\end{aligned}
$$


Proof. If $M \in \mathcal{F} \mathrm{lk}_{\infty}(A)$ then $M$ has a presentation (8) such that $\epsilon(\sigma)$ is invertible. Applying $A^{\prime}\left[F_{\mu}\right] \otimes_{A\left[F_{\mu}\right]}$ _ one obtains an exact sequence

$$
A^{\prime}\left[F_{\mu}\right] \otimes_{A\left[F_{\mu}\right]} V\left[F_{\mu}\right] \stackrel{1 \otimes \sigma}{\longrightarrow} A^{\prime}\left[F_{\mu}\right] \otimes_{A\left[F_{\mu}\right]} V\left[F_{\mu}\right] \rightarrow A^{\prime}\left[F_{\mu}\right] \otimes_{A\left[F_{\mu}\right]} M \rightarrow 0 .
$$

The naturality of the identifications $A^{\prime} \otimes_{A}\left(A \otimes_{A\left[F_{\mu}\right]} V\left[F_{\mu}\right]\right) \cong A^{\prime} \otimes_{A} V \cong$ $A^{\prime} \otimes_{A^{\prime}\left[F_{\mu}\right]}\left(A^{\prime}\left[F_{\mu}\right] \otimes_{A\left[F_{\mu}\right]} V\left[F_{\mu}\right]\right)$ implies that $\epsilon(1 \otimes \sigma)=1 \otimes \epsilon(\sigma): A^{\prime} \otimes_{A} V \rightarrow$ $A^{\prime} \otimes_{A} V$. But $1 \otimes \epsilon(\sigma)$ is an isomorphism so $1 \otimes \sigma$ is injective by Lemma 2.8. Thus $A^{\prime}\left[F_{\mu}\right] \otimes_{A\left[F_{\mu}\right]} M \in \mathcal{F} \mathrm{lk}_{\infty}\left(A^{\prime}\right)$. The argument for $\mathcal{F} \operatorname{lk}\left({ }_{-}\right)$is similar, for if $V$ is a finitely generated projective $A$-module then $A^{\prime} \otimes_{A} V$ is a finitely generated projective $A^{\prime}$-module.

\subsection{Hermitian Categories}

Recall that an involution on a ring $A$ is a map $A \rightarrow A ; a \mapsto \bar{a}$ such that $\overline{\bar{a}}=a$, $\overline{a+b}=\bar{a}+\bar{b}$ and $\overline{(a b)}=\bar{b} \bar{a}$ for all $a, b \in A$. If $A$ is a ring with involution then the category $\mathcal{F} \operatorname{lk}(A)$ can be endowed with a notion of duality. But let us begin with simpler examples:

Example 2.10. Suppose $A$ is a ring with an involution. Let $A$-Proj denote the category of finitely generated projective (left) $A$-modules. There is a duality functor defined on modules by $V \mapsto V^{*}=\operatorname{Hom}(V, A)$ for $V \in A$-Proj and on morphisms by $f \mapsto f^{*}=\ldots \circ f:\left(V^{\prime}\right)^{*} \rightarrow V^{*}$ for $f \in \operatorname{Hom}_{A}\left(V, V^{\prime}\right)$. In short,

$$
-^{*}=\operatorname{Hom}_{A}(\ldots, A): A \text {-Proj } \rightarrow A \text {-Proj. }
$$

Note that $\operatorname{Hom}_{A}(V, A)$ is a left $A$-module with

$$
(a . \xi)(x)=\xi(x) \bar{a}
$$

for all $a \in A, \xi \in \operatorname{Hom}_{A}(V, A)$ and $x \in V$.

Example 2.11. The category of finite abelian groups admits the duality functor

$$
-^{\wedge}=\operatorname{Ext}_{\mathbb{Z}}(\ldots, \mathbb{Z})
$$

A finite abelian group $M$ bears similarity to an $F$-link module in that there exists a presentation

$$
0 \rightarrow \mathbb{Z}^{n} \stackrel{\sigma}{\rightarrow} \mathbb{Z}^{n} \rightarrow M \rightarrow 0
$$

and $1 \otimes \sigma: \mathbb{Q} \otimes_{\mathbb{Z}} \mathbb{Z}^{n} \rightarrow \mathbb{Q} \otimes_{\mathbb{Z}} \mathbb{Z}^{n}$ is an isomorphism. A more explicit description of the duality functor in Example 2.11 is the following: 
Lemma 2.12. There is a natural isomorphism

$$
\operatorname{Ext}_{\mathbb{Z}}(\ldots, \mathbb{Z}) \cong \operatorname{Hom}_{\mathbb{Z}}\left(-, \frac{\mathbb{Q}}{\mathbb{Z}}\right)
$$

Proof. Suppose $M$ is a finite abelian group. The short exact sequence

$$
0 \rightarrow \mathbb{Z} \rightarrow \mathbb{Q} \rightarrow \mathbb{Q} / \mathbb{Z} \rightarrow 0
$$

gives rise to a long exact sequence

$$
0=\operatorname{Hom}(M, \mathbb{Q}) \rightarrow \operatorname{Hom}(M, \mathbb{Q} / \mathbb{Z}) \rightarrow \operatorname{Ext}(M, \mathbb{Z}) \rightarrow \operatorname{Ext}(M, \mathbb{Q}) \rightarrow \cdots
$$

which is natural in $M$. The presentation (13) implies that

$$
\operatorname{Ext}(M, \mathbb{Q})=\operatorname{Coker}\left(\operatorname{Hom}\left(\mathbb{Z}^{n}, \mathbb{Q}\right) \stackrel{\sigma^{*}}{\longrightarrow} \operatorname{Hom}\left(\mathbb{Z}^{n}, \mathbb{Q}\right)\right)=0
$$

so $\operatorname{Hom}(M, \mathbb{Q} / \mathbb{Z}) \rightarrow \operatorname{Ext}(M, \mathbb{Z})$ is an isomorphism.

The following general definition subsumes Examples 2.10 and 2.11 and the category $\mathcal{F} \operatorname{lk}(A)$ which we wish to study:

Definition 2.13. A hermitian category is a triple $\left(\mathcal{C},{ }_{-}^{*}, i\right)$ where

- $\mathcal{C}$ is an additive category,

- ${ }^{*}: \mathcal{C} \rightarrow \mathcal{C}$ is an (additive) contravariant functor and

- $\left(i_{V}\right)_{V \in \mathcal{C}}: i d \rightarrow\left({ }^{*}\right)^{*}={ }^{* *}$ is a natural isomorphism such that $i_{V}^{*} i_{V^{*}}=i d_{V^{*}}$ for all $V \in \mathcal{C}$.

The functor ${ }^{*}$ is called a duality functor. We usually abbreviate $\left(\mathcal{C},{ }^{*}, i\right)$ to $\mathcal{C}$ and identify $V$ with $V^{* *}$ via $i_{V}$. It follows from Definition 2.13 that if $\mathcal{C}$ is an abelian hermitian category then ${ }^{*}$ is an equivalence of categories and hence respects exact sequences.

If $A$ is a ring with involution then there is a unique involution on $A\left[F_{\mu}\right]$ such that $\bar{g}=g^{-1}$ for each $g \in F_{\mu}$ and such that the inclusion of $A$ in $A\left[F_{\mu}\right]$ respects the involutions. The category of finitely generated projective $A\left[F_{\mu}\right]$-modules therefore admits a duality functor as in Example 2.10.

Returning to $\mathcal{F l k}(A)$, duality is defined in a manner analogous to Example 2.11 .

Definition 2.14. Define ${ }^{\wedge}=\operatorname{Ext}_{A\left[F_{\mu}\right]}\left(\ldots, A\left[F_{\mu}\right]\right): \mathcal{F} \operatorname{lk}(A) \rightarrow \mathcal{F} \operatorname{lk}(A)$. 
Note that if $M$ has presentation (8) then $M^{\wedge}=\operatorname{Ext}_{A\left[F_{\mu}\right]}\left(M, A\left[F_{\mu}\right]\right)$ has presentation

$$
0 \rightarrow\left(V\left[F_{\mu}\right]\right)^{*} \stackrel{\sigma^{*}}{\longrightarrow}\left(V\left[F_{\mu}\right]\right)^{*} \rightarrow M^{\wedge} \rightarrow 0
$$

where $\left(V\left[F_{\mu}\right]\right)^{*}=\operatorname{Hom}_{A\left[F_{\mu}\right]}\left(V\left[F_{\mu}\right], A\left[F_{\mu}\right]\right) \cong \operatorname{Hom}_{A}(V, A)\left[F_{\mu}\right]=V^{*}\left[F_{\mu}\right]$; see Lemma 2.17 and Remark 2.18 below. The natural isomorphism

$$
V\left[F_{\mu}\right] \rightarrow\left(V\left[F_{\mu}\right]\right)^{* *}
$$

induces a natural isomorphism $i_{M}: M \rightarrow M^{\wedge \wedge}$ with $i_{M^{\wedge}}^{\wedge} i_{M^{\wedge}}=\operatorname{id}_{M^{\wedge}}$.

There is also a more explicit description of $M^{\wedge}$ which is analogous to Lemma 2.12; see Lemma 2.17 below. Unlike $\mathbb{Z}$, the $\operatorname{ring} A\left[F_{\mu}\right]$ is in general highly non-commutative so universal localization will be required.

\subsection{Universal Localization}

Let $R$ be a ring (associative with unit) and let $\Sigma$ be a set of (isomorphism classes of ) triples $\left(P_{1}, P_{0}, \sigma: P_{1} \rightarrow P_{0}\right)$ where $P_{0}$ and $P_{1}$ are finitely generated projective $R$-modules. In our application $R$ will be $A\left[F_{\mu}\right]$ and $\Sigma$ will contain the endomorphisms $\sigma: V\left[F_{\mu}\right] \rightarrow V\left[F_{\mu}\right]$ such that $V$ is finitely generated and projective as an $A$-module and $\epsilon(\sigma)$ is an automorphism of $V$.

A homomorphism $\nu: R \rightarrow S$ is said to be $\Sigma$-inverting if

$$
1 \otimes \sigma: S \otimes P_{1} \rightarrow S \otimes P_{0}
$$

is invertible for each morphism $\sigma \in \Sigma$. There exists a universal $\Sigma$-inverting homomorphism $^{5}$ which, for consistency with the other papers in the volume, will be denoted $i_{\Sigma}: R \rightarrow R_{\Sigma}$. The universal property is that every $\Sigma$ inverting homomorphism $\nu: R \rightarrow S$ may be written uniquely as a composite

$$
R \stackrel{i_{\Sigma}}{\longrightarrow} R_{\Sigma} \stackrel{\bar{\nu}}{\rightarrow} S
$$

If $R$ is commutative and each $\sigma \in \Sigma$ is an endomorphism then the localization is the ring of fractions

$$
R_{\Sigma}=R_{S}=\{p / q \mid p \in R, q \in S\}
$$

whose denominators lie in the multiplicative set $S \subseteq R$ generated by the determinants of the morphisms in $\Sigma$ :

$$
S=\left\{\prod_{i=1}^{r} \operatorname{det}\left(\sigma_{i}\right) \mid r \in \mathbb{Z}, r \geq 0, \sigma_{i} \in \Sigma\right\}
$$

${ }^{5}$ The ring $R_{\Sigma}$ was denoted $\Sigma^{-1} R$ in [51] 
More general constructions of $i_{\Sigma}$ may be found in [49, Ch4], [9, p255] or [11].

If $R=A\left[F_{\mu}\right]$ and $\Sigma$ is defined as above, the inclusion of $A\left[F_{\mu}\right]$ in $\widehat{A\left[F_{\mu}\right]}$ is $\Sigma$-inverting; see Lemmas 2.6 and 2.8 . By the universal property of $A\left[F_{\mu}\right]_{\Sigma}$ there is therefore a commutative diagram

$$
A\left[F_{\mu}\right] \stackrel{i_{\Sigma}}{\longrightarrow} A\left[F_{\mu}\right]_{\Sigma} \stackrel{\gamma}{\longrightarrow} \widehat{A\left[F_{\mu}\right]} \cong A\langle\langle X\rangle\rangle .
$$

where the natural isomorphism $\widehat{A\left[F_{\mu}\right]} \cong A\langle\langle X\rangle\rangle$ is defined as in Lemma 2.5.

The image of $\gamma$ is the ring $A_{\text {rat }}\langle\langle X\rangle\rangle$ of rational power series (see [50, §4]). If $A$ is a field or a principal ideal domain then $\gamma$ is known to be injective so $A\left[F_{\mu}\right]_{\Sigma}$ can be identified with $A_{\text {rat }}\langle\langle X\rangle\rangle$ (Cohn and Dicks [12, p416], Dicks and Sontag [13, Thm 24], Farber and Vogel [18]). In the knot theory case $\mu=1$, if $A$ is commutative then the localization is a ring of fractions

$$
A\left[F_{1}\right]_{\Sigma} \cong\left\{p / q \mid p, q \in A\left[z, z^{-1}\right], q(1) \text { is invertible }\right\} \cong A_{\text {rat }}[[x]] .
$$

and $\gamma$ is injective. However, there exist non-commutative rings $A$ such that $\gamma$ is not injective [50, Prop 1.2].

Diagram (14) and Lemma 2.6 imply:

Lemma 2.15. The localization $i_{\Sigma}: A\left[F_{\mu}\right] \rightarrow A\left[F_{\mu}\right]_{\Sigma}$ is injective.

The following is a generalization of Lemma 2.8:

Lemma 2.16. If $i_{\Sigma}: R \rightarrow R_{\Sigma}$ is injective then each $\sigma \in \Sigma$ is injective.

Proof. Suppose $\sigma: P_{1} \rightarrow P_{0}$ is in $\Sigma$. There is a commutative diagram

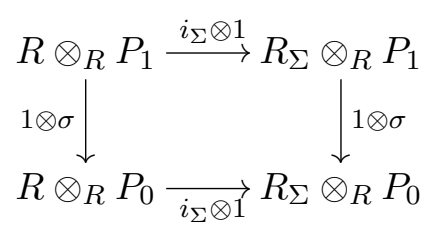

and $1 \otimes \sigma: R_{\Sigma} \otimes_{R} P_{1} \rightarrow R_{\Sigma} \otimes_{R} P_{0}$ is an isomorphism. If $i_{\Sigma}$ is injective then $1 \otimes \sigma: R \otimes_{R} P_{1} \rightarrow R \otimes_{R} P_{0}$ is also injective so $\sigma$ is injective.

Lemma 2.17. Suppose $i_{\Sigma}: R \rightarrow R_{\Sigma}$ is an injection and $M=\operatorname{Coker}(\sigma)$ with $\sigma \in \Sigma$.

1. The (right) $R$-module $M^{\wedge}=\operatorname{Ext}_{R}(M, R)$ is isomorphic to $\operatorname{Coker}\left(\sigma^{*}\right)$.

2. There is a natural isomorphism $\operatorname{Ext}_{R}(M, R) \cong \operatorname{Hom}_{R}\left(M, R_{\Sigma} / R\right)$. 
If $R$ has involution one can regard these right modules as left modules.

Proof of Lemma 2.17. (Compare Example 2.11).

1. By Lemma 2.16, the map $\sigma$ is injective so $M$ has presentation

$$
0 \rightarrow P_{1} \stackrel{\sigma}{\rightarrow} P_{0} \rightarrow M \rightarrow 0 .
$$

There is therefore an exact sequence

$$
P_{0}^{*} \stackrel{\sigma^{*}}{\longrightarrow} P_{1}^{*} \rightarrow \operatorname{Ext}_{R}(M, R) \rightarrow \operatorname{Ext}_{R}\left(P_{0}, R\right)=0
$$

2. The short exact sequence of $(R, R)$-bimodules

$$
0 \rightarrow R \rightarrow R_{\Sigma} \rightarrow R_{\Sigma} / R \rightarrow 0
$$

induces a long exact sequence of right $R$-modules

$$
\begin{aligned}
\quad \cdots \rightarrow \operatorname{Hom}_{R}\left(M, R_{\Sigma}\right) \rightarrow \operatorname{Hom}_{R}\left(M, R_{\Sigma} / R\right) & \\
& \rightarrow \operatorname{Ext}_{R}(M, R) \rightarrow \operatorname{Ext}_{R}\left(M, R_{\Sigma}\right) \rightarrow \cdots
\end{aligned}
$$

which is natural in $M$. It remains to prove that $\operatorname{Hom}_{R}\left(M, R_{\Sigma}\right)=\operatorname{Ext}_{R}\left(M, R_{\Sigma}\right)=$ 0 . The presentation (15) gives rise to the long exact sequence

$$
\begin{aligned}
0 \rightarrow \operatorname{Hom}_{R}\left(M, R_{\Sigma}\right) \rightarrow \operatorname{Hom}_{R}\left(P_{0}, R_{\Sigma}\right) \stackrel{\sigma^{*}}{\rightarrow} \operatorname{Hom}_{R}\left(P_{1}, R_{\Sigma}\right) & \\
& \rightarrow \operatorname{Ext}_{R}\left(M, R_{\Sigma}\right) \rightarrow 0 .
\end{aligned}
$$

There is a natural isomorphism

$$
\operatorname{Hom}_{R_{\Sigma}}\left(R_{\Sigma} \otimes_{R},, R_{\Sigma}\right) \rightarrow \operatorname{Hom}_{R}\left(R \otimes_{R}, R_{\Sigma}\right)
$$

induced by $i_{\Sigma}: R \rightarrow R_{\Sigma}$ and, in particular, a commutative diagram

$$
\begin{gathered}
\operatorname{Hom}_{R_{\Sigma}}\left(R_{\Sigma} \otimes_{R} P_{0}, R_{\Sigma}\right) \stackrel{(\mathrm{id} \otimes \sigma)^{*}}{\longrightarrow} \operatorname{Hom}_{R_{\Sigma}}\left(R_{\Sigma} \otimes_{R} P_{1}, R_{\Sigma}\right) \\
\stackrel{\downarrow}{\stackrel{\downarrow}{\perp}} \underset{\sigma^{*}}{\longrightarrow} \operatorname{Hom}_{R}\left(P_{1}, R_{\Sigma}\right)
\end{gathered}
$$

The upper horizontal arrow is an isomorphism since id $\otimes \sigma$ is an isomorphism so the lower horizontal arrow is also an isomorphism. Thus

$$
\operatorname{Hom}_{R}\left(M, R_{\Sigma}\right)=\operatorname{Ext}_{R}\left(M, R_{\Sigma}\right)=0
$$

as required. 
Remark 2.18. If $R \rightarrow R_{\Sigma}$ is injective and $\sigma \in \Sigma$ then the sequence $0 \rightarrow$ $P_{1} \stackrel{\sigma}{\rightarrow} P_{0} \stackrel{q}{\rightarrow} M \rightarrow 0$ is exact by Lemma 2.16. Now Lemma 2.17 gives an exact sequence

$$
P_{0}^{*} \stackrel{\sigma^{*}}{\longrightarrow} P_{1}^{*} \stackrel{q^{\prime}}{\rightarrow} \operatorname{Hom}_{R}\left(M, R_{\Sigma} / R\right) \rightarrow 0 .
$$

Let us give an explicit formula for $q^{\prime}$. There is a short exact sequence of right $R$-module chain complexes

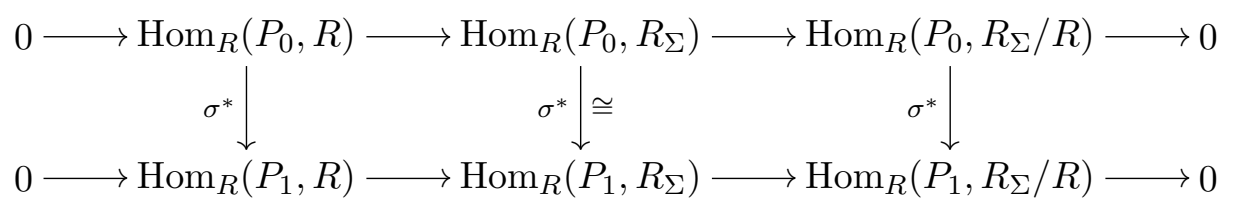

and the natural isomorphism

$$
\operatorname{Ext}_{R}(M, R)=\frac{\operatorname{Hom}_{R}\left(P_{1}, R\right)}{\operatorname{Image}\left(\sigma^{*}\right)} \longrightarrow \operatorname{Hom}_{R}\left(M, R_{\Sigma} / R\right)
$$

is inverse to the boundary map in the induced long exact homology sequence from the kernel of the right-most $\sigma^{*}$ to the cokernel of the left-most $\sigma^{*}$. Written out at length, the map $q^{\prime}: P_{1}^{*} \rightarrow \operatorname{Hom}_{R}\left(M, R_{\Sigma} / R\right)$ is the composite

$$
\begin{aligned}
\operatorname{Hom}_{R}\left(P_{1}, R\right) \rightarrow \operatorname{Hom}_{R_{\Sigma}}\left(R_{\Sigma} \otimes\right. & \left.P_{1}, R_{\Sigma}\right) \stackrel{\cong}{(\mathrm{id} \otimes \sigma)^{*}} \operatorname{Hom}_{R_{\Sigma}}\left(R_{\Sigma} \otimes P_{0}, R_{\Sigma}\right) \\
& \cong \operatorname{Hom}_{R}\left(P_{0}, R_{\Sigma}\right) \rightarrow \operatorname{Hom}_{R}\left(P_{0}, R_{\Sigma} / R\right)
\end{aligned}
$$

the image of which lies in the submodule $\operatorname{Hom}_{R}\left(M, R_{\Sigma} / R\right) \subset \operatorname{Hom}\left(P_{0}, R_{\Sigma} / R\right)$. Suppose $f \in P_{1}^{*}=\operatorname{Hom}_{R}\left(P_{1}, R\right)$ and $m \in M$. Choose $x \in P_{0}$ such that $q(x)=m$ and write $1 \otimes x \in R_{\Sigma} \otimes P_{0}$. Now

$$
q^{\prime}(f)(m)=(\mathrm{id} \otimes f)\left((\mathrm{id} \otimes \sigma)^{-1}(1 \otimes x)\right) \in R_{\Sigma} / R .
$$

Combining Lemmas 2.17 and 2.15 we have

Proposition 2.19. There is a natural isomorphism of contravariant functors

$$
\operatorname{Ext}_{A\left[F_{\mu}\right]}\left(\ldots, A\left[F_{\mu}\right]\right) \cong \operatorname{Hom}_{A\left[F_{\mu}\right]}\left(\ldots, A\left[F_{\mu}\right]_{\Sigma} / A\left[F_{\mu}\right]\right): \mathcal{F} \operatorname{lk}(A) \rightarrow \mathcal{F} \operatorname{lk}(A)
$$




\subsection{Hermitian forms and the Witt group}

As we noted in the introduction (equations (3) and (4)), the cobordism group $C_{n}\left(F_{\mu}\right)$ can be identified with a Witt group of Seifert or BlanchfieldDuval forms. Let us recall the definition of a hermitian form in a hermitian category and the appropriate definition of Witt group.

Definition 2.20. Let $\zeta=1$ or -1 . A $\zeta$-hermitian form in a hermitian category $(\mathcal{C}, *, i)$ is a pair $(V, \phi)$ where $\phi: V \rightarrow V^{*}$ and $\phi^{*} i_{V}=\zeta \phi$. If $\phi$ is an isomorphism then $\phi$ is called non-singular.

For example, in the category $\mathcal{F} \operatorname{lk}(A)$ a $\zeta$-hermitian form is an $A\left[F_{\mu}\right]$ module isomorphism $\phi: M \rightarrow M^{\wedge}=\operatorname{Hom}\left(M, A\left[F_{\mu}\right]_{\Sigma} / A\left[F_{\mu}\right]\right)$ such that $\phi^{\wedge}=\zeta \phi\left(\right.$ we suppress the natural isomorphism $\left.i_{M}\right)$.

Definition 2.21. An object $V$ in a hermitian category $(\mathcal{C}, *, i)$ is called self-dual if $V$ is isomorphic to $V^{*}$. If there exists a non-singular $\zeta$-hermitian form $(V, \phi)$ then $V$ is called $\zeta$-self-dual.

When one has a suitable notion of exact sequences in a hermitian category one can define the Witt group of the category. For simplicity, suppose $\mathcal{C}$ is a full subcategory of an abelian category $\mathcal{A}$, so that every morphism in $\mathcal{C}$ has kernel, image and cokernel in $\mathcal{A}$. Suppose further that $\mathcal{C}$ is admissible in $\mathcal{A}$ in the following sense: If there is an exact sequence $0 \rightarrow V \rightarrow V^{\prime} \rightarrow V^{\prime \prime} \rightarrow 0$ and the modules $V$ and $V^{\prime \prime}$ lie in $\mathcal{C}$ then $V^{\prime}$ lies in $\mathcal{C}$. In Section 4 below we consider a Serre subcategory of an abelian category which is defined by $V^{\prime} \in \mathcal{C}$ if and only if $V \in \mathcal{C}$ and $V^{\prime \prime} \in \mathcal{C}$. For the present we maintain greater generality; in particular an admissible subcategory $\mathcal{C}$ is not required to be an abelian category.

Definition 2.22. Let $\zeta=1$ or -1 . A non-singular $\zeta$-hermitian form $(V, \phi)$ is called metabolic if there is a submodule $L \subset V$ such that i) $L$ and $V / L$ are in $\mathcal{C}$ and ii) $L=L^{\perp}$. By definition $L^{\perp}=\operatorname{Ker}\left(j^{*} \phi: V \rightarrow L^{*}\right)$ where $j: L \rightarrow V$ is the inclusion.

Definition 2.23. The Witt group $W^{\zeta}(\mathcal{C})$ is the abelian group with one generator $[V, \phi]$ for each isomorphism class of non-singular $\zeta$-hermitian forms $(V, \phi) \in \mathcal{C}$ subject to relations

$$
\begin{cases}{\left[V^{\prime}, \phi^{\prime}\right]=[V, \phi]+\left[V^{\prime \prime}, \phi^{\prime \prime}\right],} & \text { if }\left(V^{\prime}, \phi^{\prime}\right) \cong(V, \phi) \oplus\left(V^{\prime \prime}, \phi^{\prime \prime}\right) \\ {[V, \phi]=0,} & \text { if }(V, \phi) \text { is metabolic. }\end{cases}
$$


Two forms represent the same Witt class $[V, \phi]=\left[V^{\prime}, \phi^{\prime}\right]$ if and only if there exist metabolic forms $(H, \eta)$ and $\left(H^{\prime}, \eta^{\prime}\right)$ such that

$$
(V \oplus H, \phi \oplus \eta) \cong\left(V^{\prime} \oplus H^{\prime}, \phi^{\prime} \oplus \eta^{\prime}\right)
$$

For example, a non-singular $\zeta$-hermitian form $\phi: \mathbb{Z}^{2 n} \rightarrow \mathbb{Z}^{2 n}$ in the category $\mathbb{Z}$-proj (see Example 2.10) is metabolic if there exists a summand $L \cong \mathbb{Z}^{n}$ with $\phi(L)(L)=0$.

In the category $\mathcal{F} \operatorname{lk}(A)$ a metabolizer $L$ for a form $\phi: M \rightarrow M^{\wedge}$ need not be a summand but $L$ and $M / L$ must lie in $\mathcal{F} \operatorname{lk}(A)$ and one must have $\phi(L)(L)=0$ and $\phi(x)(L) \neq 0$ if $x \notin L$. Now that the notation is defined, we repeat equation $(4)$ :

$$
C_{2 q-1}\left(F_{\mu}\right) \cong W^{(-1)^{q+1}}(\mathcal{F} \operatorname{lk}(\mathbb{Z})) . \quad(q \geq 3)
$$

\subsection{Duality-preserving functors}

A functor between hermitian categories which respects their structure is called duality-preserving. Our first examples will be functors induced by a morphism of rings with involution. Duality-preserving functors will also play an essential role in later sections (see Theorem 1.3 above and Theorem 3.19 below).

Definition 2.24. A duality-preserving functor from $\left(\mathcal{C},{ }_{-}^{*}, i\right)$ to $\left(\mathcal{D},{ }_{-}^{*}, i\right)$ is a triple $(G, \Psi, \eta)$ where

- $G: \mathcal{C} \rightarrow \mathcal{D}$ is a functor,

- $\Psi=\left(\Psi_{V}\right)_{V \in \mathcal{C}}: G\left({ }_{-}^{*}\right) \rightarrow G\left({ }_{-}\right)^{*}$ is a natural isomorphism,

- $\eta=1$ or -1

such that

$$
\Psi_{V}^{*} i_{G(V)}=\eta \Psi_{V^{*}} G\left(i_{V}\right): G(V) \rightarrow G\left(V^{*}\right)^{*}
$$

for all $V \in \mathcal{C}$.

We sometimes abbreviate $(G, \Psi, \eta)$ to $G$.

Definition 2.25. The composite of duality-preserving functors is defined by

$$
(G, \Psi, \eta) \circ\left(G^{\prime}, \Psi^{\prime}, \eta^{\prime}\right)=\left(G G^{\prime}, \Psi G\left(\Psi^{\prime}\right), \eta \eta^{\prime}\right)
$$


Example 2.26. A homomorphism $\nu: A \rightarrow A^{\prime}$ of rings with involution induces a duality-preserving functor $\left(A^{\prime} \otimes_{A_{-}}, \Pi, 1\right)$ from the category $A$-Proj of finitely generated projective $A$-modules to the category $A^{\prime}$-Proj of finitely generated projective $A^{\prime}$-modules. Explicitly

$$
\begin{aligned}
\Pi_{V}: A^{\prime} \otimes_{A}\left(V^{*}\right) & \stackrel{\cong}{\longrightarrow}\left(A^{\prime} \otimes_{A} V\right)^{*} \\
a_{1}^{\prime} \otimes \theta & \mapsto\left(a_{2}^{\prime} \otimes x \mapsto a_{2}^{\prime} \nu(\theta(x)) \overline{a_{1}^{\prime}}\right) .
\end{aligned}
$$

for all $a_{1}^{\prime}, a_{2}^{\prime} \in A^{\prime}, \theta \in V^{*}$ and $x \in V$.

We are particularly concerned with the category $\mathcal{F} \operatorname{lk}(A)$ :

Lemma 2.27. A homomorphism $\nu: A \rightarrow A^{\prime}$ of rings with involution induces a canonical duality-preserving functor

$$
\left(A^{\prime}\left[F_{\mu}\right] \otimes_{A\left[F_{\mu}\right]}, \Upsilon, 1\right): \mathcal{F} \operatorname{lk}(A) \rightarrow \mathcal{F} \operatorname{lk}\left(A^{\prime}\right) .
$$

The natural isomorphism $\Upsilon: A^{\prime}\left[F_{\mu}\right] \otimes_{A\left[F_{\mu}\right]}-^{\wedge} \rightarrow\left(A^{\prime}\left[F_{\mu}\right] \otimes_{A\left[F_{\mu}\right]}\right)^{\wedge}$ will be defined in the course of the proof.

Proof. We saw in Lemma 2.9 that there is a functor $A^{\prime}\left[F_{\mu}\right] \otimes_{A\left[F_{\mu}\right]}$ from $\mathcal{F} \operatorname{lk}(A)$ to $\mathcal{F l k}\left(A^{\prime}\right)$. The dual $M^{\wedge}$ of a module $M \in \mathcal{F} \operatorname{lk}(A)$ has presentation

$$
0 \rightarrow\left(V\left[F_{\mu}\right]\right)^{*} \stackrel{\sigma^{*}}{\longrightarrow}\left(V\left[F_{\mu}\right]\right)^{*} \rightarrow M^{\wedge} \rightarrow 0
$$

(see Remark 2.18). Applying Example 2.26 to $\nu: A\left[F_{\mu}\right] \rightarrow A^{\prime}\left[F_{\mu}\right]$ one obtains a natural isomorphism

$$
\Pi_{V\left[F_{\mu}\right]}: A^{\prime}\left[F_{\mu}\right] \otimes_{A\left[F_{\mu}\right]}\left(V\left[F_{\mu}\right]\right)^{*} \cong\left(A^{\prime}\left[F_{\mu}\right] \otimes_{A\left[F_{\mu}\right]} V\left[F_{\mu}\right]\right)^{*}
$$

and hence a commutative diagram

$$
\begin{aligned}
& 0 \rightarrow A^{\prime}\left[F_{\mu}\right] \otimes_{A\left[F_{\mu}\right]}\left(V\left[F_{\mu}\right]\right)^{*} \rightarrow A^{\prime}\left[F_{\mu}\right] \otimes_{A\left[F_{\mu}\right]}\left(V\left[F_{\mu}\right]\right)^{*} \longrightarrow A^{\prime}\left[F_{\mu}\right] \otimes_{A\left[F_{\mu}\right]} M^{\wedge} \longrightarrow 0 \\
& \downarrow \cong \quad \downarrow \cong \quad \Upsilon_{M} \\
& 0 \rightarrow\left(A^{\prime}\left[F_{\mu}\right] \otimes_{A\left[F_{\mu}\right]} V\left[F_{\mu}\right]\right)^{*} \rightarrow\left(A^{\prime}\left[F_{\mu}\right] \otimes_{A\left[F_{\mu}\right]} V\left[F_{\mu}\right]\right)^{*} \rightarrow\left(A^{\prime}\left[F_{\mu}\right] \otimes_{A\left[F_{\mu}\right]} M\right)^{\wedge} \rightarrow 0 \text {. }
\end{aligned}
$$

We must check that the induced isomorphism

$$
\Upsilon_{M}: A^{\prime}\left[F_{\mu}\right] \otimes_{A\left[F_{\mu}\right]} M^{\wedge} \rightarrow\left(A^{\prime}\left[F_{\mu}\right] \otimes_{A\left[F_{\mu}\right]} M\right)^{\wedge}
$$

is independent of the choice of presentation $\sigma$ and that $\Upsilon$ is natural with respect to $M$. If $C$ denotes the chain complex $V\left[F_{\mu}\right] \stackrel{\sigma}{\rightarrow} V\left[F_{\mu}\right]$ and $C^{\prime}$ denotes 
an alternative choice of resolution for $M$, say $C^{\prime}=\left(V^{\prime}\left[F_{\mu}\right] \stackrel{\sigma^{\prime}}{\rightarrow} V^{\prime}\left[F_{\mu}\right]\right)$ then the identity map id $: M \rightarrow M$ lifts to a chain equivalence $C \rightarrow C^{\prime}$. The naturality of $\Pi$ in Example 2.26 implies that the diagram

$$
\begin{gathered}
A^{\prime}\left[F_{\mu}\right] \otimes C^{*} \rightarrow A^{\prime}\left[F_{\mu}\right] \otimes\left(C^{\prime}\right)^{*} \\
\downarrow \\
\downarrow \\
\left(A^{\prime}\left[F_{\mu}\right] \otimes C\right)^{*} \rightarrow\left(A^{\prime}\left[F_{\mu}\right] \otimes C^{\prime}\right)^{*}
\end{gathered}
$$

commutes. The horizontal arrows induce the identity map on $A^{\prime}\left[F_{\mu}\right] \otimes$ $M^{\wedge}$ and $\left(A^{\prime}\left[F_{\mu}\right] \otimes M\right)^{\wedge}$ respectively, so the vertical arrows induce the same map $\Upsilon_{M}$. The naturality of $\Upsilon$ follows similarly from the naturality of the transformation $\Pi$ in Example 2.26.

Remark 2.28. Identifying $M^{\wedge}$ with $\operatorname{Hom}\left(M, A\left[F_{\mu}\right]_{\Sigma} / A\left[F_{\mu}\right]\right)$ by Proposition 2.19, an explicit formula for $\Upsilon_{M}$ is

$$
\begin{aligned}
\Upsilon_{M}: A^{\prime}\left[F_{\mu}\right] \otimes_{A\left[F_{\mu}\right]} \operatorname{Hom}\left(M, \frac{A\left[F_{\mu}\right]_{\Sigma}}{A\left[F_{\mu}\right]}\right) & \rightarrow \operatorname{Hom}\left(A^{\prime}\left[F_{\mu}\right] \otimes_{A\left[F_{\mu}\right]} M, \frac{A^{\prime}\left[F_{\mu}\right]_{\Sigma}}{A^{\prime}\left[F_{\mu}\right]}\right) \\
a_{1}^{\prime} \otimes \theta & \mapsto\left(a_{2}^{\prime} \otimes m \mapsto a_{2}^{\prime} \nu(\theta(m)) \overline{a_{1}^{\prime}}\right)
\end{aligned}
$$

We conclude this section by noting the effect of duality-preserving functor on Witt groups:

Lemma 2.29. A duality-preserving functor $(G, \Psi, \eta): \mathcal{C} \rightarrow \mathcal{D}$ which respects exact sequences induces a homomorphism of Witt groups

$$
\begin{aligned}
G: W^{\zeta}(\mathcal{C}) & \rightarrow W^{\zeta \eta}(\mathcal{D}) \\
{[V, \phi] } & \mapsto\left[G(V), \Psi_{V} G(\phi)\right]
\end{aligned}
$$

Proof. See for example [51, p41-42].

\section{Intrinsic Invariants}

In [51] the author defined invariants of the cobordism group $C_{2 q-1}\left(F_{\mu}\right)$ of $F_{\mu}$-links using the identification (3) due to Ko [27] and Mio [38] of $C_{2 q-1}\left(F_{\mu}\right)$ with a Witt group of $\mu$-component Seifert forms, denoted $W^{(-1)^{q}}(\mathcal{S}$ ei $(\mathbb{Z}))$ below $(q \geq 3)$. To distinguish $F_{\mu}$-links one first chooses a Seifert surface for each and then computes invariants of the associated Seifert forms.

In the present section we define $F_{\mu}$-link cobordism invariants via Duval's identification $C_{2 q-1}\left(F_{\mu}\right) \cong W^{(-1)^{q}}(\mathcal{F} \operatorname{lk}(\mathbb{Z}))$. The definitions will parallel those in [51] and we shall prove in Section 6 that the invariants obtained 
are equivalent. Whereas Seifert forms are convenient for computing the invariants in explicit examples, the Blanchfield-Duval form has the advantage that it is defined without making a choice of Seifert surface.

\subsection{Overview}

Let $\zeta=1$ or -1 . The inclusion $\mathbb{Z} \subset \mathbb{Q}$ induces a duality-preserving functor $\mathcal{F} \operatorname{lk}(\mathbb{Z}) \rightarrow \mathcal{F} \operatorname{lk}(\mathbb{Q})$ (see Lemma 2.27 above) and hence a homomorphism of Witt groups

$$
W^{\zeta}(\mathcal{F} \operatorname{lk}(\mathbb{Z})) \rightarrow W^{\zeta}(\mathcal{F} \operatorname{lk}(\mathbb{Q})) .
$$

It follows from Theorem 1.3 that (21) is an injection; see the proof of Corollary 3.3 below. We proceed to compute $W^{\zeta}(\mathcal{F l k}(\mathbb{Q}))$ in three steps, which were outlined in the introduction. We list them again here in more detail:

1. Devissage. We prove that $\mathcal{F} \operatorname{lk}(\mathbb{Q})$ is an abelian category with ascending and descending chain conditions. Recall that a module $M \in$ $\mathcal{F} \operatorname{lk}(\mathbb{Q})$ is called simple (or irreducible) if $M \neq 0$ and there are no submodules of $M$ in $\mathcal{F} l \mathrm{k}(\mathbb{Q})$ other than 0 and $M$. If $M$ is a simple module then $\left.\mathcal{F} \operatorname{lk}(\mathbb{Q})\right|_{M} \subset \mathcal{F} \operatorname{lk}(\mathbb{Q})$ denotes the full subcategory in which the objects are direct sums of copies of $M$. Recall that $M$ is called $\zeta$-selfdual if there is an isomorphism $b: M \rightarrow M^{\wedge}$ such that $b^{\wedge}=\zeta b$. We obtain, by "hermitian devissage", the decomposition

$$
W^{\zeta}(\mathcal{F} \operatorname{lk}(\mathbb{Q})) \cong \bigoplus W^{\zeta}\left(\left.\mathcal{F} \operatorname{lk}(\mathbb{Q})\right|_{M}\right)
$$

with one summand for each isomorphism class of $\zeta$-self-dual simple $F_{\mu}$-link modules $M$. Let $p_{M}$ denote the projection of $W^{\zeta}(\mathcal{F} \operatorname{lk}(\mathbb{Q}))$ onto $W^{\zeta}\left(\left.\mathcal{F} \operatorname{lk}(\mathbb{Q})\right|_{M}\right)$.

2. Morita equivalence. For each $\zeta$-self-dual simple module $M$ we choose a non-singular $\zeta$-hermitian form $b: M \rightarrow M^{\wedge}$. We obtain by hermitian Morita equivalence an isomorphism

$$
\Theta_{M, b}: W^{\zeta}\left(\left.\mathcal{F} \operatorname{lk}(\mathbb{Q})\right|_{M}\right) \rightarrow W^{1}(E)
$$

where $E=\operatorname{End}_{\mathbb{Q}\left[F_{\mu}\right]} M$ is the endomorphism ring of $M$ and is endowed with the involution $f \mapsto b^{-1} f^{\wedge} b$. By Schur's Lemma $E$ is a division ring and, as we discuss, $E$ turns out to be of finite dimension over $\mathbb{Q}$.

3. We recall from the literature invariants of each group $W^{1}(E)$. In most cases some combination of dimension modulo 2 , signatures, discriminant and Hasse-Witt invariant are sufficient to distinguish the elements 
of $W^{1}(E)$ (see the table (29) below). One class of division algebra with involution requires a secondary invariant, such as the Lewis $\theta$, which is defined only if all the other invariants vanish.

Let us make two remarks about the modules $M$ which appear in item 1 . Firstly, every simple module $M \in \mathcal{F} l \mathrm{k}(\mathbb{Q})$ such that $M \cong M^{\wedge}$ is either 1 -self-dual or (-1)-self-dual (or both). Secondly, $M$ is both 1-self-dual and $(-1)$-self-dual if and only if the involution $f \mapsto b^{-1} f^{\wedge} b$ induced on $E=$ $\operatorname{End}_{\mathbb{Q}\left[F_{\mu}\right]}(M)$ is not the identity map for some (and therefore every) $\zeta$-selfdual form $b: M \rightarrow M^{\wedge}$. See lemmas 5.5 and 5.6 of [51] for details.

Example 3.1. In the knot theory case $\mu=1$ we can add simplifying remarks to each of the three steps:

1. A simple self-dual module $M \in \mathcal{F} \operatorname{lk}(\mathbb{Q})$ may be written

$$
M=\mathbb{Q}\left[z, z^{-1}\right] /(p)
$$

where $p$ is an irreducible polynomial and $\left(p\left(z^{-1}\right)\right)=(p(z)) \triangleleft \mathbb{Q}\left[z, z^{-1}\right]$.

2. The endomorphism ring $E=\operatorname{End}_{\mathbb{Q}\left[z, z^{-1}\right]}(M)$ may also be written as a quotient $\mathbb{Q}\left[z, z^{-1}\right] /(p)$ and is an algebraic number field of finite dimension over $\mathbb{Q}$. The involution on $E$ is given by $z \mapsto z^{-1}$ and does not depend on the choice of form $b$.

Setting aside the case $M=\mathbb{Q}\left[z, z^{-1}\right] /(1+z)$, the involution on $E$ is not the identity so every self-dual $M$ is both 1-self-dual and (-1)-self-dual. The exceptional module $M=\mathbb{Q}\left[z, z^{-1}\right] /(1+z)$ is only 1 -self-dual but plays little role since -1 is not a root of any polynomial $p \in \mathbb{Z}\left[z, z^{-1}\right]$ such that $p(1)= \pm 1$. In other words, the projection of $W^{1}(\mathcal{F} l \mathrm{k}(\mathbb{Z}))$ on this exceptional summand of $W^{1}(\mathcal{F l}(\mathbb{Q}))$ is zero.

3. As discussed in 2., one need only consider the Witt groups $W^{1}(E)$ of number fields with non-trivial involution, or in other words, hermitian forms over number fields. The dimension modulo 2 , signatures and discriminant are sufficient to distinguish the elements of $W^{1}(E)$.

Equation (1) can be derived as a consequence of this analysis.

Returning to the general case $\mu \geq 1$ and putting together steps 1-3. we obtain the following restatement of Theorem 1.1.

Theorem 3.2. Let $\zeta=1$ or -1 . An element $\alpha \in W^{\zeta}(\mathcal{F} \operatorname{lk}(\mathbb{Q}))$ is equal to zero if and only if for each finite-dimensional $\zeta$-self-dual simple $F_{\mu}$-link 
module $M$ and non-singular $\zeta$-hermitian form $b: M \rightarrow M^{\wedge}$, the dimension modulo 2, the signatures, the discriminant, the Hasse-Witt invariant and the Lewis $\theta$-invariant of

$$
\Theta_{M, b} p_{M}(\alpha) \in W^{1}\left(\operatorname{End}_{\mathbb{Q}\left[F_{\mu}\right]} M\right)
$$

are trivial (if defined).

Note that if the invariants corresponding to one form $b: M \rightarrow M^{\wedge}$ are trivial then $p_{M}(\alpha)=0$ so the invariants are trivial for any other choice $b^{\prime}: M \rightarrow M^{\wedge}$. We now restate Corollary 1.2:

Corollary 3.3. Suppose $\left(L^{0}, \theta^{0}\right)$ and $\left(L^{1}, \theta^{1}\right)$ are $(2 q-1)$-dimensional $F_{\mu^{-}}$ links, where $q>1$. Let $\overline{X_{i}}$ denote the free cover of the complement of $L_{i}$, let $N_{i}=H_{q}\left(\overline{X_{i}}\right) /(\mathbb{Z}$-torsion $)$ and let $\phi^{i}: N_{i} \rightarrow \operatorname{Hom}\left(N_{i}, \mathbb{Z}\left[F_{\mu}\right]_{\Sigma} / \mathbb{Z}\left[F_{\mu}\right]\right)$ denote the Blanchfield-Duval form for $\left(L^{i}, \theta^{i}\right)$.

The $F_{\mu}$-links $\left(L^{0}, \theta^{0}\right)$ and $\left(L^{1}, \theta^{1}\right)$ are cobordant if and only if for each finite-dimensional $\zeta$-self-dual simple $F_{\mu}$-link module $M$ and each non-singular $\zeta$-hermitian form $b: M \rightarrow M^{\wedge}$, the dimension modulo 2 , the signatures, the discriminant, the Hasse-Witt invariant and the Lewis $\theta$-invariant of

$$
\Theta_{M, b} p_{M}\left[\mathbb{Q} \otimes_{\mathbb{Z}}\left(N^{0} \oplus N^{1}, \phi^{0} \oplus-\phi^{1}\right)\right] \in W^{1}\left(\operatorname{End}_{\mathbb{Q}\left[F_{\mu}\right]} M\right)
$$

are trivial (if defined).

Proof. We deduce Corollary 3.3 from Theorem 1.1 (=Theorem 3.2) and Theorem 1.3. As we remarked in the introduction, Corollary 3.3 also follows from Theorem 1.4 and Theorem B of [51].

Proposition 5.7 below says that the duality-preserving functor

$$
(B, \Phi,-1): \mathcal{S} \operatorname{ei}(A) \rightarrow \mathcal{F} \operatorname{lk}(A)
$$

of Section 5 respects coefficient change so there is a commutative diagram

$$
\begin{gathered}
W^{(-1)^{q}}(\mathcal{S} \operatorname{ei}(\mathbb{Z})) \stackrel{B}{\longrightarrow} W^{(-1)^{q+1}}(\mathcal{F} \operatorname{lk}(\mathbb{Z})) \\
\downarrow \\
W^{(-1)^{q}}(\mathcal{S} \operatorname{ei}(\mathbb{Q})) \underset{B}{\longrightarrow} W^{(-1)^{q+1}}(\mathcal{F} \operatorname{lk}(\mathbb{Q})) .
\end{gathered}
$$

The category $\mathcal{S} \operatorname{ei}(A)$ is defined in Section 4.1. The lower horizontal map in (24) is an isomorphism by Theorem 1.3 and the upper horizontal map is an isomorphism by (3) and (4) above (see also Remark 5.6). It is easy to prove that the left hand vertical map is an injection (see for example Lemma 11.1 of [51]). Thus the right-hand vertical map is also an injection. Corollary 3.3 therefore follows from Theorem 3.2 


\subsection{Step 1: Devissage}

Let us briefly recall some definitions. If $\mathcal{A}$ is an additive category and $M$ is an object in $\mathcal{A}$ the symbol $\left.\mathcal{A}\right|_{M}$ denotes the full subcategory such that $N \in \mathcal{A}$ if and only if $N$ is a summand of some finite direct sum of copies of $M$. If $\mathcal{A}$ is a hermitian category and $M$ is self-dual then $\left.\mathcal{A}\right|_{M}$ is a hermitian subcategory.

Suppose now that $\mathcal{A}$ is an abelian category. A non-zero module $M$ in $\mathcal{A}$ is called simple (or irreducible) if there are no submodules of $M$ in $\mathcal{A}$ other than 0 and $M$. The category $\mathcal{A}$ has both ascending and descending chain conditions if and only if every module $M$ in $\mathcal{A}$ has a finite composition series

$$
0=M_{0} \subset M_{1} \subset M_{2} \subset M_{3} \subset \cdots \subset M_{s}=M
$$

where $M_{i} / M_{i-1}$ is simple for $i=1, \cdots, s$. If $M$ is simple then every module in $\left.\mathcal{A}\right|_{M}$ is a direct sum of copies of $M$. Let $\zeta=1$ or -1 . If $(\mathcal{A}, *, i)$ is a hermitian category then a module $M$ is called $\zeta$-self-dual if there is an isomorphism $\phi: M \rightarrow M^{*}$ such that $\phi^{*} i_{M}=\zeta \phi$.

The general decomposition theorem we need is the following:

Theorem 3.4 (Devissage). Suppose $\mathcal{A}$ is an abelian hermitian category with ascending and descending chain conditions. There is an isomorphism of Witt groups

$$
W^{\zeta}(\mathcal{C}) \cong \bigoplus W^{\zeta}\left(\left.\mathcal{C}\right|_{M}\right)
$$

with one summand for each isomorphism class of simple $\zeta$-self-dual modules in $\mathcal{C}$.

Proof. See [51, Theorem 5.3] or [41].

To prove equation (22) it therefore suffices to show:

Proposition 3.5. If $k$ is a (commutative) field then the category $\mathcal{F l k}(k)$ is an abelian category with ascending and descending chain conditions.

We take coefficients in a field for simplicity. With little extra work one can show that Proposition 3.5 holds when $k$ is replaced by any semi-simple Artinian ring. See also Remark 3.18 below.

Caveat 3.6. When $\mu \geq 2$ a simple module in the category $\mathcal{F} l \mathrm{k}(k)$ is not a simple module in the category of $k\left[F_{\mu}\right]$-modules. 
We prove Proposition 3.5 as follows: We first show that $\mathcal{F l k}(k)$ is a subcategory of the category $\mathcal{T}_{k\left[F_{\mu}\right]}$ of "torsion" modules which P.M.Cohn introduced and proved to be an abelian category with ascending and descending chain conditions (see Proposition 3.13 below). After giving details of Cohn's work, we conclude the proof of Proposition 3.5 by checking that $\mathcal{F} \operatorname{lk}(k)$ is closed under direct sums and that the kernel and cokernel of every morphism in $\mathcal{F} l \mathrm{k}(k)$ again lie in $\mathcal{F l k}(k)$.

\subsubsection{Firs and torsion modules}

To describe Cohn's results we must state some properties of the group ring $k\left[F_{\mu}\right]$.

Definition 3.7. A ring $R$ has invariant basis number (IBN) if $R^{n} \cong R^{m}$ implies $n=m$. In other words $R$ has IBN if every finitely generated free left $R$-module has unique rank.

The existence of the augmentation $\epsilon: k\left[F_{\mu}\right] \rightarrow k$ implies that $k\left[F_{\mu}\right]$ has IBN for if $k\left[F_{\mu}\right]^{n} \cong k\left[F_{\mu}\right]^{m}$ then

$$
k^{n} \cong k \otimes_{k\left[F_{\mu}\right]} k\left[F_{\mu}\right]^{n} \cong k \otimes_{k\left[F_{\mu}\right]} k\left[F_{\mu}\right]^{m} \cong k^{m}
$$

so $m=n$.

Note that if $R$ has IBN then one can use the duality functor $\operatorname{Hom}(\ldots, R)$ to prove that finitely generated free right $R$-modules also have unique rank.

Definition 3.8. An associative ring $R$ is called a free ideal ring (fir) if $R$ has IBN, every left ideal in $R$ is a free left $R$-module and every right ideal is a free right $R$-module.

Cohn showed in [8, Corollary 3] that if $k$ is a field then the group ring $k\left[F_{\mu}\right]$ of the free group $F_{\mu}$ is a fir.

If $R$ is a fir then every submodule of a free $R$-module is free (Cohn [10, p71]). Hence every $R$-module has a presentation $0 \rightarrow F_{1} \rightarrow F_{0} \rightarrow M \rightarrow 0$ where $F_{1}$ and $F_{0}$ are free.

Definition 3.9. If an $R$-module $M$ has a presentation

$$
0 \rightarrow R^{n} \stackrel{\sigma}{\rightarrow} R^{m} \stackrel{p}{\rightarrow} M \rightarrow 0
$$

the Euler characteristic of $M$ is $\chi(M)=m-n$. 
If a finite presentation exists the Euler characteristic is independent of the choice of presentation by Schanuel's Lemma. Note also that an exact sequence $0 \rightarrow M \rightarrow M^{\prime} \rightarrow M^{\prime \prime} \rightarrow 0$ of finitely presented modules implies the equation $\chi\left(M^{\prime}\right)=\chi(M)+\chi\left(M^{\prime \prime}\right)$ (compare the diagram (27) below).

If $R$ is a fir then the category of finitely presented $R$-modules (and $R$ module maps) is an abelian category; in other words direct sums of finitely presented modules are finitely presented and the cokernel and kernel of a map between finitely presented modules are finitely presented. In fact, if $R$ is any ring such that every finitely generated one-sided ideal is finitely related then the finitely presented $R$-modules form an abelian category (e.g. Cohn [10, p554-556]).

Definition 3.10. A morphism $\sigma: R^{n} \rightarrow R^{n}$ between free left $R$-modules is called full if every factorization

$$
R^{n} \underset{\sigma_{2}}{\stackrel{\sigma}{\longrightarrow} \underset{\sigma_{1}}{\longrightarrow}} R^{n}
$$

where $F$ is a free module has $\operatorname{Rank}(F) \geq n$.

Lemma 3.11. Suppose $R$ is a fir and $M$ is a finitely presented $R$-module with $\chi(M)=0$. The following are equivalent:

1. In every finite presentation (26) of $M$, the map $\sigma$ is full.

2. There exists a presentation (26) such that $\sigma$ is full.

3. $\chi(N) \geq 0$ for all finitely generated submodules $N$ of $M$.

4. $\chi(M / N) \leq 0$ for all finitely generated submodules $N$ of $M$.

Proof. The implication $1 \Rightarrow 2$ is immediate. To show 2 implies 3 , suppose we are given a presentation (26) such that $m=n$ and $\sigma$ is full. If $N$ is a finitely generated submodule of $M$ then $\sigma\left(R^{n}\right) \subset p^{-1}(N) \subset R^{n}$. Now $p^{-1}(N)$ is a free module because $R$ is a fir and $p^{-1}(N)$ has rank at least $n$ since $\sigma$ is full. The exact sequence

$$
0 \rightarrow R^{n} \rightarrow p^{-1}(N) \rightarrow N \rightarrow 0
$$

implies that $\chi(N) \geq 0$. This completes the proof that 2 implies 3 .

The equation $\chi(M)=\chi(N)+\chi(M / N)$ implies that 3 and 4 are equivalent so we can conclude the proof of the Lemma by showing that 3 implies 1 . The equation $\chi(M)=0$ says that every finite presentation (26) has $m=n$. 
We must prove that $\sigma$ is full. Suppose $\sigma$ can be written as a composite $R^{n} \stackrel{\sigma_{2}}{\longrightarrow} R^{k} \stackrel{\sigma_{1}}{\longrightarrow} R^{n}$. We aim to show $k \geq n$. Now $\sigma\left(R^{n}\right)$ and $\sigma_{1}\left(R^{k}\right)$ are free modules since $R$ is a fir and

$$
\frac{\sigma_{1}\left(R^{k}\right)}{\sigma\left(R^{n}\right)} \subseteq \frac{R^{n}}{\sigma\left(R^{n}\right)}=M
$$

By statement $3, \chi\left(\sigma_{1}\left(R^{k}\right) / \sigma\left(R^{n}\right)\right) \geq 0$ so $\operatorname{Rank}\left(\sigma_{1}\left(R^{k}\right)\right) \geq \operatorname{Rank}\left(\sigma\left(R^{n}\right)\right)=$ $n$. Thus $k \geq n$ and hence $\sigma$ is full.

A module which satisfies the equivalent conditions in Lemma 3.11 is called a torsion module. The symbol $\mathcal{T}_{R}$ denote the category of torsion modules and $R$-module maps.

Lemma 3.12. (Cohn $[10, p 166]$ ) If $0 \rightarrow M \rightarrow M^{\prime} \rightarrow M^{\prime \prime} \rightarrow 0$ is an exact sequence and $M$ and $M^{\prime \prime}$ are torsion modules then $M^{\prime}$ is a torsion module. In particular a direct sum of torsion modules is again a torsion module.

Proof. If $M$ and $M^{\prime \prime}$ are finitely presented then $M^{\prime}$ is also finitely presented (compare (27) below). Since $\chi(M)=\chi\left(M^{\prime \prime}\right)=0$ we have $\chi\left(M^{\prime}\right)=0$. Now if $N \leq M^{\prime}$ is finitely generated it suffices by Lemma 3.11 to show that $\chi(N) \geq 0$. Note first that $\chi(N)=\chi(N \cap M)+\chi(N /(N \cap M))$. Now $N \cap M \leq M$ so $\chi(N \cap M) \geq 0$ and $N /(N \cap M) \cong(N+M) / M \leq M^{\prime \prime}$ so $\chi(N /(N \cap M)) \geq 0$. Thus $\chi(N) \geq 0$.

Proposition 3.13. (Cohn [10, p167,234]) Suppose $R$ is a fir.

1. The category $\mathcal{T}_{R}$ of torsion modules is an abelian category.

2. Every module in $\mathcal{T}_{R}$ has a finite composition series (25) in $\mathcal{T}_{R}$.

Proof. To establish statement 1 , it suffices to show that if $M$ and $M^{\prime}$ lie in $\mathcal{T}_{R}$ then $M \oplus M^{\prime} \in \mathcal{T}_{R}$ and the kernel and cokernel of every morphism $f: M \rightarrow M^{\prime}$ are in $\mathcal{T}_{R}$. Lemma 3.12 gives $M \oplus M^{\prime} \in \mathcal{T}_{R}$. Suppose then that $f: M \rightarrow M^{\prime}$ is an $R$-module morphism. Since the finitely presented $R$-modules are an abelian category the kernel, image and cokernel of $f$ are finitely presented. Now $f(M)$ is a submodule of $M^{\prime}$ and a quotient module of $M$ so $\chi(f(M)) \geq 0$ and $\chi(f(M)) \leq 0$ by Lemma 3.11. Thus $\chi(f(M))=0$ and it follows that $\chi(\operatorname{Ker}(f))=0$ and $\chi(\operatorname{Coker}(f))=0$. Every finitely generated submodule $N \leq \operatorname{Ker}(f)$ is a submodule of $M$ so $\chi(N) \geq 0$ and hence $\operatorname{Ker}(f) \in \mathcal{T}_{R}$. Similarly every quotient $N^{\prime}$ of $\operatorname{Coker}(f)$ is a quotient of $M^{\prime}$ and hence has $\chi\left(N^{\prime}\right) \leq 0$. Thus $\operatorname{Coker}(f) \in \mathcal{T}_{R}$ also. 
To prove part 2. of Proposition 3.13 we note first that it is sufficient to check the ascending chain condition. Indeed, if $M \in \mathcal{T}_{R}$ then $M^{\wedge}=$ $\operatorname{Ext}_{R}(M, R)$ is a torsion right $R$-module and a descending chain

$$
M=M_{0} \supseteq M_{1} \supseteq M_{2} \supseteq \cdots
$$

in $\mathcal{T}_{R}$ gives rise to an ascending chain

$$
\left(\frac{M}{M_{0}}\right)^{\wedge} \subseteq\left(\frac{M}{M_{1}}\right)^{\wedge} \subseteq\left(\frac{M}{M_{2}}\right)^{\wedge} \subseteq \cdots
$$

In fact Cohn showed that a larger class of modules, the finitely related bound modules, have the ascending chain condition.

Definition 3.14. An $R$-module $M$ is bound if $\operatorname{Hom}(M, R)=0$.

Lemma 3.15. Every torsion module over a fir is bound.

Proof. If $M$ is an $R$-module and $\theta: M \rightarrow R$ then $\theta(M)$ is a free module so $M \cong \operatorname{Ker}(\theta) \oplus \theta(M)$. Now $\theta(M)$ is a quotient module of $M$ so if $M$ is a torsion module then $\chi(\theta(M)) \leq 0$. It follows that $\theta(M)=0$ so $\theta=0$. Thus $\operatorname{Hom}_{R}(M, R)=0$.

Lemma 3.16. (Cohn [10, p231]) If $R$ is a fir and $M$ is a finitely related $R$-module then every bound submodule of $M$ is finitely presented.

Proof. There is an exact sequence $0 \rightarrow R^{n} \rightarrow F \stackrel{p}{\rightarrow} M \rightarrow 0$ where $F$ is a free $R$-module. A submodule $B \leq M$ has presentation

$$
0 \rightarrow R^{n} \rightarrow p^{-1}(B) \rightarrow B \rightarrow 0
$$

and $p^{-1}(B)$ is a free module since $R$ is a fir. The image of $R^{n}$ is contained in a finitely generated summand of $p^{-1}(B)$ so $B$ is a direct sum of a free module and a finitely presented module. If $B$ is bound then $B$ does not have any non-zero free summand so $B$ itself is finitely presented.

Thus every torsion submodule of a torsion module $M$ is finitely generated so we have the ascending chain condition on torsion submodules. It follows that $\mathcal{T}_{R}$ has both ascending and descending chain conditions and the proof of Proposition 3.13 is complete.

We are now in a position to deduce Proposition 3.5.

Lemma 3.17. The category $\mathcal{F} \operatorname{lk}(k)$ is a full subcategory of the category $\mathcal{T}_{k\left[F_{\mu}\right]}$ of torsion modules. 
Proof. It suffices to show that every module in $\mathcal{F} \operatorname{lk}(k)$ is a torsion module. By definition, a module in $\mathcal{F} \operatorname{lk}(k)$ has a presentation (8) where $\epsilon(\sigma)$ is an isomorphism. Since $\epsilon(\sigma)$ is full we can deduce that $\sigma$ is full since each factorization of $\sigma$ induces a corresponding factorization of $\epsilon(\sigma)$.

Proof of Proposition 3.5. Since $\mathcal{T}_{k\left[F_{\mu}\right]}$ has ascending and descending chain conditions and $\mathcal{F} \mathrm{lk}(k) \subset \mathcal{T}_{k\left[F_{\mu}\right]}$ we need only show that $\mathcal{F} \mathrm{lk}(k)$ is an abelian category.

Suppose $M$ and $M^{\prime}$ are in $\mathcal{F} \operatorname{lk}(k)$. It follows directly from the definition of $\mathcal{F} \operatorname{lk}(k)$ that $M \oplus M^{\prime} \in \mathcal{F} \operatorname{lk}(k)$. We must show that the kernel and cokernel of each map $f: M \rightarrow M^{\prime}$ lie in $\mathcal{F l k}(k)$. By Proposition 3.13 the kernel, image and cokernel all lie in $\mathcal{T}_{k\left[F_{\mu}\right]}$ so

$$
\chi(\operatorname{Ker}(f))=\chi(f(M))=\chi(\operatorname{Coker}(f))=0 .
$$

After choosing presentations $\sigma$ and $\sigma^{\prime}$ for $\operatorname{Ker}(f)$ and $f(M)$ respectively one can fill in the dotted arrows below to obtain a commutative diagram with exact rows and exact columns:

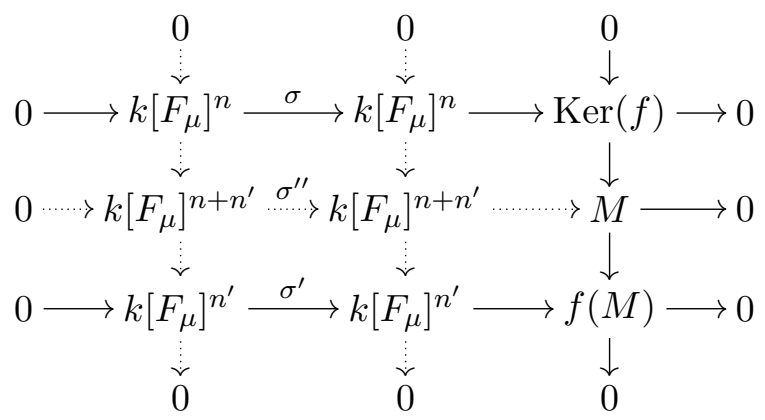

The map $\sigma^{\prime \prime}$ is given by $\left(\begin{array}{cc}\sigma & \tau \\ 0 & \sigma^{\prime}\end{array}\right)$ for some $\tau$. Since $M \in \mathcal{F} \operatorname{lk}(k)$, the augmentation $\epsilon\left(\sigma^{\prime \prime}\right)$ is an isomorphism by Lemma 2.3 above. It follows that $\epsilon(\sigma)$ and $\epsilon\left(\sigma^{\prime \prime}\right)$ are isomorphisms and hence $\operatorname{Ker}(f)$ and $f(M)$ are in $\mathcal{F} \operatorname{lk}(k)$. The same argument, applied to the exact sequence $0 \rightarrow f(M) \rightarrow M^{\prime} \rightarrow$ $\operatorname{Coker}(f) \rightarrow 0$ shows that $\operatorname{Coker}(f)$ is also in $\mathcal{F} l \mathrm{k}(k)$.

Remark 3.18. The arguments above can be adapted to generalize Propositions 3.13 and 3.5 as follows. Suppose every finitely generated one-sided ideal of a ring $R$ is a projective module (i.e. $R$ is semi-hereditary). Suppose $S$ is a ring with the property that $S^{n} \oplus P \cong S^{n}$ implies $P=0$ (i.e. $S$ is weakly finite) and $\nu: R \rightarrow S$ is a ring homomorphism. The Grothendieck 
group $K_{0}(S)$ admits a partial order in which $x \leq y$ if and only $y-x$ lies in the positive cone

$$
\{[P] \mid P \in S \text {-Proj }\} \subset K_{0}(S) .
$$

Now the following category $\mathcal{T}_{\nu}$ is an abelian category: An $R$-module $M$ lies in $\mathcal{T}_{\nu}$ if $M$ has a finite presentation by projective modules $0 \rightarrow P_{1} \stackrel{\sigma}{\rightarrow} P_{0} \rightarrow$ $M \rightarrow 0$ such that $1 \otimes \sigma: S \otimes_{R} P_{1} \rightarrow S \otimes P_{0}$ is full with respect to the partial order on $K_{0}(S)$. The subcategory of modules for which $1 \otimes \sigma$ is invertible is also an abelian category. Under the additional hypotheses that all the one-sided ideals of $R$ are projective modules (i.e. $R$ is hereditary) and that the equation $S \otimes_{R} P=0$ implies $P=0$ for projective $R$-modules $P$, one can also conclude that these abelian categories have ascending and descending chain conditions. One recovers Proposition 3.13 when $R$ is a fir by setting $\nu=\mathrm{id}: R \rightarrow R$ and one recovers Proposition 3.5 by setting $\nu=\epsilon: k\left[F_{\mu}\right] \rightarrow k$.

\subsection{Step 2: Morita Equivalence}

Having reduced $W^{\zeta}(\mathcal{F} \operatorname{lk}(\mathbb{Q}))$ to a direct sum of Witt groups $W^{\zeta}\left(\left.\mathcal{F} \operatorname{lk}(\mathbb{Q})\right|_{M}\right)$ where $M$ is simple and $\zeta$-self-dual (see equation (22)) we pass next from $W^{\zeta}\left(\left.\mathcal{F} l \mathrm{k}(\mathbb{Q})\right|_{M}\right)$ to the Witt group $W^{1}\left(\operatorname{End}_{\mathbb{Q}\left[F_{\mu}\right]} M\right)$ of the endomorphism ring of $M$.

Recall that if $\mathcal{C}$ is an additive category then an object in $\left.\mathcal{C}\right|_{M}$ is a summand of a direct sum of copies of $M$. The general theorem we employ in this section is the following:

Theorem 3.19 (Hermitian Morita Equivalence). Let $\eta=+1$ or -1 . Suppose that $b: M \rightarrow M^{*}$ is a non-singular $\eta$-hermitian form in a hermitian category $\mathcal{C}$, and assume further that every idempotent endomorphism in the hermitian subcategory $\left.\mathcal{C}\right|_{M}$ splits. Let $E=\operatorname{End}_{\mathcal{C}} M$ be endowed with the involution $f \mapsto \bar{f}=b^{-1} f^{*} b$. Then there is an equivalence of hermitian categories

$$
\Theta_{M, b}=\left(\operatorname{Hom}\left(M,{ }_{-}\right), \Omega^{b}, \eta\right):\left.\mathcal{C}\right|_{M} \rightarrow E \text {-Proj }
$$

where for $\left.N \in \mathcal{C}\right|_{M}$ the map $\Omega_{N}^{b}(\gamma)=\left(\alpha \mapsto \eta b^{-1} \alpha^{*} \gamma\right)$ is the composite of natural isomorphisms

$$
\begin{aligned}
\operatorname{Hom}_{\mathcal{C}}\left(M, N^{*}\right) & \rightarrow \operatorname{Hom}_{\mathcal{C}}(N, M) \rightarrow \operatorname{Hom}_{E}\left(\operatorname{Hom}_{\mathcal{C}}(M, N), E\right) \\
\gamma & \mapsto b^{-1} \gamma^{*} ; \quad \delta \mapsto(\alpha \mapsto \overline{\delta \alpha}) .
\end{aligned}
$$

Proof. See [51, Theorem 4.7], [26, §I.9,ch.II] or [41]. 
The following is a corollary of Theorem 3.19 and Lemma 2.29.

Corollary 3.20. If $M$ is a simple module in $\mathcal{F} \operatorname{lk}(k)$ and $b: M \rightarrow M^{\wedge}$ is a non-singular $\zeta$-hermitian form then the duality-preserving functor $\Theta_{M, b}$ of Theorem 3.19 induces an isomorphism of Witt groups

$$
\begin{aligned}
W^{\zeta}\left(\left.\mathcal{F} \operatorname{lk}(k)\right|_{M}\right) & \rightarrow W^{1}\left(\operatorname{End}_{k\left[F_{\mu}\right]} M\right) \\
{[N, \phi] } & \mapsto\left[\operatorname{Hom}(M, N), \Omega_{N}^{b} \phi_{*}\right]
\end{aligned}
$$

where $\phi_{*}: \operatorname{Hom}(M, N) \rightarrow \operatorname{Hom}\left(M, N^{*}\right)$ and

$$
\left(\Omega_{N}^{b} \phi_{*}\right)(\alpha)(\beta)=\zeta b^{-1} \beta^{*} \phi \alpha \in \operatorname{End}_{k\left[F_{\mu}\right]} M
$$

for all $\alpha, \beta \in \operatorname{Hom}(M, N)$. Equation (22) implies that

$$
W^{\zeta}(\mathcal{F} \operatorname{lk}(k)) \cong \bigoplus_{M} W^{1}\left(\operatorname{End}_{k\left[F_{\mu}\right]} M\right) .
$$

with one summand for each isomorphism class of $\zeta$-self-dual simple $F_{\mu}$-link modules $M$.

Proof. Every exact sequence in $\left.\mathcal{F} \operatorname{lk}(k)\right|_{M}$ splits, so $\Theta_{M, b}$ is exact and hence induces a morphism of Witt groups

$$
W^{\zeta}\left(\left.\mathcal{F l k}(k)\right|_{M}\right) \rightarrow W^{1}\left(\operatorname{End}_{k\left[F_{\mu}\right]}(M)\right) .
$$

Since $\Theta_{M, b}$ is an equivalence of hermitian categories it follows by Lemma A.2 of Appendix A that the induced map of Witt groups is an isomorphism.

Equation (23) above is a special case of corollary 3.20 so the following proposition completes step 2:

Proposition 3.21. The endomorphism ring of every module in $\mathcal{F} \operatorname{lk}(k)$ is of finite dimension over $k$.

Proposition 3.21 follows from Theorem 5.17 and Lemma 5.34 below. Theorem 5.17 can be considered analogous to the geometric fact that one can choose a Seifert surface for an $F_{\mu}$-link. Since the homology of a Seifert surface is finite-dimensional the endomorphism ring of the associated Seifert module is finite-dimensional. Using chapter 12 of [51], part 2. of Lemma 6.1 and part 2. of Lemma 6.2 we can also deduce that every division ring with involution which is finite-dimensional over $\mathbb{Q}$ arises as

$$
\left(\operatorname{End}_{\mathbb{Q}\left[F_{\mu}\right]} M, f \mapsto \bar{f}=b^{-1} f^{\wedge} b\right)
$$


for some pair $(M, b)$ where $M$ is a simple module in $\mathcal{F} \operatorname{lk}(\mathbb{Q})$.

The proofs of the results cited in the previous paragraph do not use Proposition 3.21 (i.e. the arguments presented are not circular). However, the spirit of this section is to define invariants of $F_{\mu}$-links by studying the category $\mathcal{F} \operatorname{lk}(\mathbb{Q})$ directly so we desire a proof of Theorem 3.21 which avoids any choice of Seifert surface or Seifert module. One such proof is due to Lewin [34]. In a subsequent paper we shall give a proof which applies when $k\left[F_{\mu}\right]$ is replaced by a wider class of rings.

Before leaving the subject of Morita equivalence we pause to note the following "naturality" statement which we will need in Section 6 to prove Theorem 1.4. The reader may refer to equation (19) for the definition of composition for duality-preserving functors.

Proposition 3.22. Suppose $\left(G, \Psi, \eta^{\prime}\right): \mathcal{C} \rightarrow \mathcal{D}$ is a duality-preserving functor between hermitian categories and $b: M \rightarrow M^{*}$ is an $\eta$-hermitian form in $\mathcal{C}$. Let $E=\operatorname{End}_{\mathcal{C}}(M)$ and let $E^{\prime}=\operatorname{End}_{\mathcal{D}} G(M)$. The following diagram of duality-preserving functors commutes up to natural isomorphism:

$$
\begin{aligned}
& \begin{array}{c}
\left.\left.\mathcal{C}\right|_{M} \stackrel{\left(G, \Psi, \eta^{\prime}\right)}{\longrightarrow} \mathcal{D}\right|_{G(M)} \\
\left(\operatorname{Hom}(M, \ldots), \Omega^{b}, \eta\right) \downarrow \\
\downarrow\left(\operatorname{Hom}(G(M), \ldots), \Omega^{\eta^{\prime} \Psi G(b)}, \eta \eta^{\prime}\right)
\end{array} \\
& E \text {-Proj } \underset{\left(E^{\prime} \otimes_{E}, \Pi, 1\right)}{\longrightarrow} E^{\prime} \text {-Proj. }
\end{aligned}
$$

Proof. See Section A.2 of Appendix A.

Corollary 3.23. If $G$ is exact then the following square also commutes:

$$
\begin{gathered}
W^{\zeta}\left(\left.\mathcal{C}\right|_{M}\right) \stackrel{G}{\longrightarrow} W^{\zeta \eta^{\prime}}\left(\left.\mathcal{D}\right|_{G(M)}\right) \\
\left.\Theta_{M, b}\right\rfloor \cong \\
\cong \downarrow \Theta_{G(M), \eta^{\prime} \Psi G(b)} \\
W^{\zeta \eta}(E) \stackrel{G}{\longrightarrow} W^{\zeta \eta}\left(E^{\prime}\right) .
\end{gathered}
$$

\subsection{Step 3: Invariants}

Equation (22) leads one to consider invariants to distinguish Witt classes of forms over division algebras $E$ of finite dimension over $\mathbb{Q}$. Such division algebras are well understood (see Albert [1, p149,p161], Scharlau [48] or our earlier summary in $[51, \S 11.1,11.2])$.

One considers five distinct classes of division algebras with involution. Firstly a division algebra $E$ may be commutative or non-commutative. Secondly, if $I$ is an involution on $E$ let $\operatorname{Fix}(I)=\{a \in E \mid I(a)=a\}$. The 
involution is said to be "of the first kind" if Fix $(I)$ contains the center $K=Z(E)$ of $E$. Otherwise, the involution is "of the second kind". Finally, one of these four classes is further partitioned. A non-commutative division algebra with involution of the first kind is necessarily a quaternion algebra, with presentation $K\left\langle i, j \mid i^{2}=a, j^{2}=b, i j=-j i\right\rangle$ for some number field $K$ and some elements $a, b \in K$. If $\operatorname{Fix}(I)=Z(E)=K$ then the involution is called "standard". On the other hand if Fix $(I)$ strictly larger than $K$ then the involution is called "non-standard".

Table (29) below lists sufficient invariants to distinguish the Witt classes of forms over each class of division algebras with involution. The symbol $m$ (2) denotes dimension modulo 2. The letter $\sigma$ signifies all signature invariants (if any) each of which takes values in $\mathbb{Z}$. The discriminant $\Delta$ is the determinant with a possible sign adjustment and takes values in the group of "square classes"

$$
\frac{\operatorname{Fix}(I) \cap K}{\left\{a I(a) \mid a \in K^{\bullet}\right\}}
$$

where $K$ is the center of $E$ and $K^{\bullet}=K \backslash 0$.

\begin{tabular}{|c|l|l|l|}
\hline Kind & Commutative? & Involution & Invariants \\
\hline $1 s t$ & Yes & (Trivial) & $m(2), \sigma, \Delta, c$ \\
\hline $1 s t$ & No & Standard & $m(2), \sigma$ \\
\hline $1 s t$ & No & Non-standard & $m(2), \sigma, \Delta, \theta$ \\
\hline $2 n d$ & Yes & (Non-trivial) & $m(2), \sigma, \Delta$ \\
\hline $2 n d$ & No & (Non-trivial) & $m(2), \sigma, \Delta$ \\
\hline
\end{tabular}

Two symbols in the table have not yet been mentioned. The Hasse-Witt invariant, $c$, which appears in the first row takes values in a direct sum of copies of $\{1,-1\}$, one copy for each prime of the number field $K$. Finally, if $E$ is a quaternion algebra with non-standard involution of the first kind then the local-global principle fails and one requires a secondary invariant such as the Lewis $\theta$ which is defined if all the other invariants vanish. The value group for $\theta$ is the quotient $\{1,-1\}^{S} / \sim$ where $S$ is the set of primes $\mathfrak{p}$ of $K$ such that the completion $E_{\mathfrak{p}}$ is a division algebra and the relation $\sim$ identifies each element $\left\{\epsilon_{\mathfrak{p}}\right\}_{\mathfrak{p} \in S}$ with its antipode $\left\{-\epsilon_{\mathfrak{p}}\right\}_{\mathfrak{p} \in S}$.

\section{Seifert forms}

In this section we describe algebraic structures arising in the study of a Seifert surface of an $F_{\mu}$-link. We define in Section 4.1 a category $\mathcal{S e i}_{\infty}(A)$ 
of "Seifert modules" and a full subcategory $\mathcal{S} \operatorname{ei}(A) \subset \mathcal{S} \operatorname{ei}_{\infty}(A)$ in which the objects are finitely generated and projective as $A$-modules. The category $\mathcal{S}$ ei $(A)$ was denoted $\left(P_{\mu}-A\right)$-Proj in [51].

In Section 4.2 we prove that every $F_{\mu}$-link module is also a Seifert module in a canonical way (cf Farber [16]) and obtain a "forgetful" functor

$$
U: \mathcal{F} \mathrm{lk}_{\infty}(A) \rightarrow \mathcal{S} \mathrm{ei}_{\infty}(A)
$$

The image of $\mathcal{F} \operatorname{lk}(A)$ is usually not contained in $\mathcal{S} \operatorname{ei}(A)$ which explains our motivation for introducing $\mathcal{S}_{\mathrm{ei}}(A)$. We construct later (Section 5) a functor $B$ from $\mathcal{S} \operatorname{ei}(A)$ to $\mathcal{F} \operatorname{lk}(A)$ which extends in an obvious way to a functor $B: \mathcal{S e i}_{\infty}(A) \rightarrow \mathcal{F} \mathrm{lk}_{\infty}(A)$ and is left adjoint to $U$.

In Section 4.3 we put hermitian structure on $\mathcal{S} \operatorname{ei}(A)$. We will see in Section 6 below that the functor $B$ induces an isomorphism of Witt groups $W^{\zeta}(\mathcal{S} \operatorname{ei}(A)) \rightarrow W^{-\zeta}(\mathcal{F} \operatorname{lk}(A))$ when $A$ is semi-simple and Artinian and, in particular, when $A=\mathbb{Q}$.

\subsection{Seifert modules}

Suppose $V$ is a finite-dimensional vector space over a field $k$ and $\alpha: V \rightarrow V$ is an endomorphism. A time-honoured technique in linear algebra regards the pair $(V, \alpha)$ as a module over a polynomial ring $k[s]$ in which the action of $s$ on $V$ is given by $\alpha$. Equivalently, $(V, \alpha)$ is a representation of $\mathbb{Z}[s]$ in the category of finite-dimensional vector spaces over $k$. We shall use the words "module" and "representation" interchangeably.

Given a Seifert surface $U^{n+1} \subset S^{n+2}$ for a knot $S^{n} \subset S^{n+2}$, small translations in the directions normal to $U$ induce homomorphisms

$$
f^{+}, f^{-}: H_{i}(U) \rightarrow H_{i}\left(S^{n+2} \backslash U\right)
$$

Using Alexander duality one finds that $f^{+}-f^{-}$is an isomorphism for $i \neq$ $0, n+1$, so $H_{i}(U)$ is endowed with an endomorphism $\left(f^{+}-f^{-}\right)^{-1} f^{+}$and may therefore be regarded as a representation of a polynomial ring $\mathbb{Z}[s]$. The homology of a Seifert surface for a $\mu$-component boundary link has, in addition to the endomorphism $\left(f^{+}-f^{-}\right)^{-1} f^{+}$, a system of $\mu$ orthogonal idempotents which express the component structure of the Seifert surface. Following Farber [17] (see also [51]) we regard $H_{i}(U)$ as a representation of 
the ring

$$
\begin{aligned}
P_{\mu} & \left.=\mathbb{Z}\left\langle s, \pi_{1}, \cdots, \pi_{\mu}\right| \pi_{i}^{2}=\pi_{i} ; \pi_{i} \pi_{j}=0 \text { for } i \neq j ; \sum_{i=1}^{\mu} \pi_{i}=1\right\rangle . \\
& \cong \mathbb{Z}[s] * \mathbb{Z}\left(\prod_{\mu} \mathbb{Z}\right) .
\end{aligned}
$$

$P_{\mu}$ is also the path ring of a quiver which we illustrate in the case $\mu=2$ :

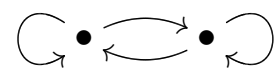

Notation 4.1. Let $\mathcal{S} \mathrm{ei}_{\infty}(A)$ denote the category of representations of $P_{\mu}$ by $A$-modules. An object in $\mathcal{S} \mathrm{ei}_{\infty}(A)$ (called a Seifert module) is a pair $(V, \rho)$ where $V$ is an $A$-module and $\rho: P_{\mu} \rightarrow \operatorname{End}_{A}(V)$ is a ring homomorphism.

Let $\mathcal{S}$ ei $(A)$ denote the category of representations of $P_{\mu}$ by finitely generated projective $A$-modules. In other words, $\mathcal{S} \operatorname{ei}(A)$ is the full subcategory of pairs $(V, \rho)$ such that $V$ is finitely generated and projective.

We sometimes omit $\rho$, confusing an element of $P_{\mu}$ with its image in $\operatorname{End}_{A}(V)$.

\subsection{Seifert structure on $F_{\mu}$-link modules}

Every $F_{\mu}$-link module has a canonical Seifert module structure which we describe next. In fact $\mathcal{F} \mathrm{k}_{\infty}(A)$ can be regarded as a full subcategory of $\mathcal{S} \mathrm{ei}_{\infty}(A)$. Note however that a module in $\mathcal{F} \operatorname{lk}(A)$ is in general neither finitely generated nor projective as an $A$-module (e.g. see Lemma 2.3 above) and therefore does not lie in $\mathcal{S}$ ei $(A)$.

If $M \in \mathcal{F} \mathrm{lk}_{\infty}(A)$ then Lemma 2.3 implies that the $A$-module map

$$
\begin{aligned}
\gamma: M^{\oplus \mu} & \rightarrow M \\
\left(m_{1}, \cdots, m_{\mu}\right) & \mapsto \sum_{i=1}^{\mu}\left(1-z_{i}\right) m_{i}
\end{aligned}
$$

is an isomorphism. Let $p_{i}$ denote the projection of $M^{\oplus \mu}$ onto its $i$ th component and let

$$
\begin{gathered}
\omega: M^{\oplus \mu} \rightarrow M \\
\left(m_{1}, \cdots, m_{\mu}\right) \mapsto \sum_{i=1}^{\mu} m_{i}
\end{gathered}
$$


denote addition. Define $\rho: P_{\mu} \rightarrow \operatorname{End}_{A} M$ by

$$
\begin{aligned}
& \rho\left(\pi_{i}\right)=\gamma p_{i} \gamma^{-1} \\
& \rho(s)=\omega \gamma^{-1} .
\end{aligned}
$$

We denote by $U(M)$ the $A$-module $M$ with the Seifert module structure $\rho$. As remarked in the introduction, in the case $\mu=1$ the Seifert module structure $\rho: P_{\mu} \rightarrow \operatorname{End}_{A}(M)$ can be described more simply by the equation

$$
\rho(s)=(1-z)^{-1} .
$$

The following lemma says that $U: \mathcal{F} \mathrm{lk}_{\infty}(A) \rightarrow \mathcal{S}_{\mathrm{ei}}(A)$ is a full and faithful functor, so $\mathcal{F} \mathrm{lk}_{\infty}(A)$ can be regarded as a full subcategory of $\mathcal{S} \mathrm{ei}_{\infty}(A)$.

Lemma 4.2. An A-module morphism $f: M \rightarrow M^{\prime}$ between $F_{\mu}$-link modules $M$ and $M^{\prime}$ is an $A\left[F_{\mu}\right]$-module morphism if and only if $f$ is a morphism of Seifert modules. In other words, $f \in \operatorname{Hom}_{\mathcal{S e i}_{\infty}(A)}\left(U(M), U\left(M^{\prime}\right)\right)$ if and only if $f \in \operatorname{Hom}_{\mathcal{F} \mathrm{k}_{\infty}(A)}\left(M, M^{\prime}\right)$.

Proof. If $f: M \rightarrow M^{\prime}$ is an $A\left[F_{\mu}\right]$-module morphism then the diagram

$$
\begin{gathered}
M^{\oplus \mu} \stackrel{f^{\oplus \mu}}{\longrightarrow} M^{\prime \oplus \mu} \\
\downarrow \downarrow \\
M \underset{f}{\downarrow} M^{\prime}
\end{gathered}
$$

is commutative. Conversely, if the diagram (31) commutes then the equation $f\left(\left(1-z_{i}\right) x\right)=\left(1-z_{i}\right) f(x)$ holds for each $x \in M$ and $i=1, \cdots, \mu$, so $f\left(z_{i} x\right)=z_{i} f(x)$ for each $i$ and hence $f$ is an $A\left[F_{\mu}\right]$-module morphism.

It remains to show that (31) commutes if and only if $f$ is a Seifert morphism. If (31) commutes then

$$
f\left(\gamma p_{i} \gamma^{-1} x\right)=\gamma p_{i} \gamma^{-1} f(x) \text { and } \quad f\left(\omega \gamma^{-1} x\right)=\omega \gamma^{-1} f(x)
$$

for each $x \in M$ so $f$ is a Seifert morphism. Conversely, suppose $f$ is a Seifert morphism. Now

$$
\gamma^{-1}=\left(\begin{array}{c}
\omega p_{1} \gamma^{-1} \\
\omega p_{2} \gamma^{-1} \\
\vdots \\
\omega p_{\mu} \gamma^{-1}
\end{array}\right)=\left(\begin{array}{c}
\left(\omega \gamma^{-1}\right)\left(\gamma p_{1} \gamma^{-1}\right) \\
\left(\omega \gamma^{-1}\right)\left(\gamma p_{2} \gamma^{-1}\right) \\
\vdots \\
\left(\omega \gamma^{-1}\right)\left(\gamma p_{\mu} \gamma^{-1}\right)
\end{array}\right): M \rightarrow M^{\oplus \mu}
$$

so the diagram (31) commutes. 


\subsection{Seifert forms}

Let us make $\mathcal{S}$ ei $(A)$ a hermitian category, assuming $A$ is a ring with involution. Recall that if $V$ is a finitely generated projective left $A$-module then $V^{*}=\operatorname{Hom}(V, A)$ is a left $A$-module with $(a . \theta)(x)=\theta(x) \bar{a}$ for all $a \in A$, $\theta \in V^{*}$ and $x \in V$.

If $(V, \rho)$ is an object in $\mathcal{S e i}(A)$ define $(V, \rho)^{*}=\left(V^{*}, \rho^{*}\right)$ where

$$
\rho^{*}\left(\pi_{i}\right)=\rho\left(\pi_{i}\right)^{*}: V^{*} \rightarrow V^{*} \quad \text { and } \quad \rho^{*}(s)=1-\rho(s)^{*}: V^{*} \rightarrow V^{*} .
$$

It is easy to see that if $f: V \rightarrow V^{\prime}$ is a morphism in $\mathcal{S}$ ei $(A)$ then the dual $f^{*}:\left(V^{\prime}\right)^{*} \rightarrow V^{*}$ again lies in $\mathcal{S}$ ei $(A)$. Equivalently, if one gives the ring $P_{\mu}$ the involution defined by $\bar{s}=1-s$ and $\overline{\pi_{i}}=\pi_{i}$ for each $i$ then $\rho^{*}: P_{\mu} \rightarrow \operatorname{End}\left(V^{*}\right)$ is given by $\rho^{*}(r)(\theta)(x)=\theta(\rho(\bar{r}) x)$ for all $x \in V, \theta \in V^{*}$ and $r \in P_{\mu}$.

If $V=H_{q}\left(U^{2 q}\right) /$ torsion then the intersection form $\phi: V \rightarrow V^{*}$ is a morphism in $\mathcal{S}$ ei $(\mathbb{Z})$. In other words, the intersection form respects the projections $\pi_{i}$ and respects the endomorphism $\left(f^{+}-f^{-}\right)^{-1} f^{+}$(see section 4.1) in the sense that

$$
\phi\left(\left(f^{+}-f^{-}\right)^{-1} f^{+} x\right)(y)=\phi(x)\left(\left(1-\left(f^{+}-f^{-}\right)^{-1} f^{+}\right) y\right) .
$$

for all $x, y \in V$. Furthermore, $\phi$ is an isomorphism by Poincaré duality and is $(-1)^{q}$-hermitian. This form $\phi$ will be called the Seifert form associated to a Seifert surface. Kervaire [25, p94] or Lemma 3.31 of [51] shows that by an elementary change of variables, this form $\phi$ is equivalent to the Seifert matrix of linking numbers more commonly encountered in knot theory.

By Ko [27] and Mio [38] (see also Lemma 3.31 of [51]), the association of this form $\phi$ to a Seifert surface induces the isomorphism

$$
C_{2 q-1}\left(F_{\mu}\right) \cong W^{(-1)^{q}}(\mathcal{S} \operatorname{ei}(\mathbb{Z})) \quad(q \geq 3) .
$$

mentioned in the introduction. Every $(-1)^{q}$-hermitian Seifert form is associated to some $2 q$-dimensional Seifert surface. Although there are many possible Seifert surfaces for a given $F_{\mu}$-link, all are cobordant and the corresponding Seifert forms lie in the same Witt class.

In $[51]$ the author applied to $\mathcal{S}$ ei $(\mathbb{Q})$ the steps 1-3. described in Section 3.1 obtaining explicit invariants to distinguish $F_{\mu}$-link cobordism classes. Although the Blanchfield-Duval form is more intrinsic, the advantage of the Seifert form is that it is easier to compute the numerical invariants. For illustration, we treat a worked example: 
Example 4.3. Setting $\mu=2$, consider the Seifert module $V=\mathbb{Z}^{6}$ with the endomorphism $s$ and (-1)-hermitian form $\phi$ given by

$$
s=\left(\begin{array}{rrrr|rr}
1 & 0 & 1 & 0 & 0 & 0 \\
0 & 1 & -1 & -1 & -1 & 0 \\
0 & 1 & 0 & 0 & 0 & -1 \\
0 & 0 & 0 & 0 & 0 & 0 \\
\hline 0 & 1 & 0 & 0 & 1 & -1 \\
0 & 0 & 1 & 0 & 1 & 0
\end{array}\right) \quad \text { and } \quad \phi=\left(\begin{array}{rrrr|rr}
0 & 0 & 0 & 1 & 0 & 0 \\
0 & 0 & -1 & 0 & 0 & 0 \\
0 & 1 & 0 & 0 & 0 & 0 \\
-1 & 0 & 0 & 0 & 0 & 0 \\
\hline 0 & 0 & 0 & 0 & 0 & -1 \\
0 & 0 & 0 & 0 & 1 & 0
\end{array}\right)
$$

The horizontal and vertical lines indicate the component structure of the Seifert form. In other words, $\pi_{1}$ projects onto the span of the first four basis elements while $\pi_{2}$ projects onto the span of the last two. The corresponding Seifert matrix of linking numbers is

$$
\phi s=\left(\begin{array}{rrrr|rr}
0 & 0 & 0 & 0 & 0 & 0 \\
0 & -1 & 0 & 0 & 0 & 1 \\
0 & 1 & -1 & -1 & -1 & 0 \\
-1 & 0 & -1 & 0 & 0 & 0 \\
\hline 0 & 0 & -1 & 0 & -1 & 0 \\
0 & 1 & 0 & 0 & 1 & -1
\end{array}\right)
$$

but we shall work directly with $s$ and $\phi$.

The first step is to pass from $\mathbb{Z}$ to $\mathbb{Q}$ so we regard the entries in the matrices as rational numbers. Devissage is next; let $e_{1}, \cdots, e_{6}$ denote the standard basis of $\mathbb{Q}^{6}$. Now $\mathbb{Q} e_{1}$ is $s$-invariant and $\phi\left(e_{1}\right)\left(e_{1}\right)=0$ so our Seifert form is Witt-equivalent to the induced form on $e_{1}^{\perp} / \mathbb{Q} e_{1} \cong \mathbb{Q}\left\{e_{2}, e_{3}, e_{5}, e_{6}\right\}$. We have reduced $s$ and $\phi$ to

$$
s^{\prime}=\left(\begin{array}{rr|rr}
1 & -1 & -1 & 0 \\
1 & 0 & 0 & -1 \\
\hline 1 & 0 & 1 & -1 \\
0 & 1 & 1 & 0
\end{array}\right) \quad \text { and } \quad \phi^{\prime}=\left(\begin{array}{rr|rr}
0 & -1 & 0 & 0 \\
1 & 0 & 0 & 0 \\
\hline 0 & 0 & 0 & -1 \\
0 & 0 & 1 & 0
\end{array}\right)
$$

The two-dimensional representation (over $\mathbb{Q}$ ) of $\mathbb{Z}[s]$ given by the matrix $r=\left(\begin{array}{cc}1 & -1 \\ 1 & 0\end{array}\right)$ is simple (=irreducible) since there do not exist eigenvalues in $\mathbb{Q}$. It follows that the Seifert module $V^{\prime}=\mathbb{Q}^{4}$ with the action $s^{\prime}$ and $\pi_{1}, \pi_{2}$ shown is simple. The devissage process is therefore complete.

Turning to the Morita equivalence step, the endomorphism ring of this module $V^{\prime}$ has $\mathbb{Q}$-basis consisting of the identity and $\left(\begin{array}{l|l}r & 0 \\ \hline 0 & r\end{array}\right)$. The minimum polynomial of $r$ is $x^{2}-x+1$ so $\operatorname{End}_{\mathcal{S e i}(\mathbb{Q})}\left(V^{\prime}\right)$ is isomorphic to $\mathbb{Q}(\sqrt{-3})$. 
We may choose $b=-\phi^{\prime}: V^{\prime} \rightarrow\left(V^{\prime}\right)^{*}$. It is easy to verify that the involution $f \mapsto b^{-1} f^{*} b$ is not the identity map so it must send $\sqrt{-3}$ to $-\sqrt{-3}$. Morita equivalence sends the form $\phi^{\prime}: V^{\prime} \rightarrow V^{* *}$ to the composite

$$
\begin{aligned}
\operatorname{Hom}_{\mathcal{S e i}(\mathbb{Q})}\left(V^{\prime}, V^{\prime}\right) \stackrel{\phi^{\prime}}{\longrightarrow} \operatorname{Hom}_{\mathcal{S e i}(\mathbb{Q})}\left(V^{\prime}, V^{\prime *}\right) \\
\stackrel{\Omega_{V^{\prime}}^{b}}{\longrightarrow} \operatorname{Hom}_{\mathbb{Q}(\sqrt{-3})}\left(\operatorname{Hom}\left(V^{\prime}, V^{\prime *}\right), \operatorname{Hom}\left(V^{\prime}, V^{\prime}\right)\right)
\end{aligned}
$$

which is given by

$$
\Omega_{V^{\prime}}^{b} \phi_{*}^{\prime}(\alpha)(\beta)=-b^{-1} \beta^{*} \phi^{\prime} \alpha=\bar{\beta} \alpha .
$$

for $\alpha, \beta \in \operatorname{Hom}\left(V^{\prime}, V^{\prime}\right)$. This form may be written $\langle 1\rangle$ as a form over $\mathbb{Q}(\sqrt{-3})$.

Reading the fourth line of the table (29), an element of the Witt group $W(\mathbb{Q}(\sqrt{-3}))$ for non-trivial involution is determined by signatures and discriminant (and rank modulo 2 if there are no signatures). Up to complex conjugation there is precisely one embedding of $\mathbb{Q}(\sqrt{-3})$ in $\mathbb{C}$ (with the complex conjugate involution), so there is in fact one signature, which takes value $1 \in \mathbb{Z}$ with our choice of $b$. The discriminant is

$$
1 \in \frac{\mathbb{Q} \backslash 0}{\mathbb{Q}(\sqrt{-3}) \overline{\mathbb{Q}(\sqrt{-3})}}=\frac{\mathbb{Q} \backslash 0}{\left\{a^{2}+3 b^{2} \mid a, b \in \mathbb{Q}\right\}} .
$$

\section{The Covering construction}

In this section we introduce a functor $B: \mathcal{S} \operatorname{ei}_{\infty}(A) \rightarrow \mathcal{F} \mathrm{lk}_{\infty}(A)$ which is the algebraic analogue of the geometric construction of the free cover of an $F_{\mu}$-link complement from a Seifert surface. (illustrated in Figure 1 on page 150). The restriction of $B$ to $\mathcal{S}$ ei $(A)$ takes values in $\mathcal{F} \operatorname{lk}(A)$ and extends to a duality-preserving functor

$$
(B, \Phi,-1): \mathcal{S} \operatorname{ei}(A) \rightarrow \mathcal{F} \operatorname{lk}(A)
$$

which is natural in $A$ (see Propositions 5.4 and 5.7).

We show that $B: \mathcal{S} \operatorname{ei}_{\infty}(A) \rightarrow \mathcal{F} \mathrm{lk}_{\infty}(A)$ is left adjoint to the full and faithful functor $U: \mathcal{F} \mathrm{lk}_{\infty}(A) \rightarrow \mathcal{S} \operatorname{ei}_{\infty}(A)$; in other words, among functors $\mathcal{S} \operatorname{ei}_{\infty}(A) \rightarrow \mathcal{F} \mathrm{lk}_{\infty}(A)$, the geometrically motivated functor $B$ satisfies a universal property with respect to $U$ (see Definition 5.8). In particular there is a natural transformation $\theta_{V}: V \rightarrow U B(V)$ for $V \in \mathcal{S} \operatorname{ei}(A)$ and a natural isomorphism $\psi_{M}: B U(M) \rightarrow M$ for $M \in \mathcal{F} \mathrm{lk}_{\infty}(A)$. The reader is 
referred to chapter 3 of Borceux [4] or chapter IV of Mac Lane [35] for a detailed treatment of adjoint functors.

We use the adjunction in Section 5.3 to show that the covering construction $B: \mathcal{S} \operatorname{ei}_{\infty}(A) \rightarrow \mathcal{F} \mathrm{lk}_{\infty}(A)$ is equivalent to a universal localization $\mathcal{S} \operatorname{ei}_{\infty}(A) \rightarrow \mathcal{S} \operatorname{ei}_{\infty}(A) / \mathcal{P} \operatorname{rim}_{\infty}(A)$ of categories. We describe the structure of the "primitive" modules $V \in \mathcal{P} \operatorname{rim}_{\infty}(A)$ in Section 5.5 and outline a construction of the quotient category in Section 5.6.

We show in Sections 5.3 and 5.4 that $B: \mathcal{S} \operatorname{ei}(A) \rightarrow \mathcal{F} \operatorname{lk}(A)$ is equivalent to a localization $\mathcal{S} \operatorname{ei}(A) \rightarrow \mathcal{S} \operatorname{ei}(A) / \mathcal{P} \operatorname{rim}_{\infty}(A)$ of hermitian categories. In the case where $A$ is a semi-simple Artinian ring we simplify the descriptions of the quotient and primitive modules in Section 5.7.

\subsection{Definition}

To simplify notation in this section and Section 5.2, we suppress the symbol $\rho$ which appears in the definition of a Seifert module $(V, \rho)$, identifying an element $r \in P_{\mu}$ with $\rho(r) \in \operatorname{End}_{A}(V)$. We shall extend Seifert structure from an $A$-module $V$ to the induced module $A\left[F_{\mu}\right] \otimes_{A} V$ by $s(\alpha \otimes v)=\alpha \otimes s(v)$ and $\pi_{i}(\alpha \otimes v)=\alpha \otimes \pi_{i}(v)$ for $\alpha \in A\left[F_{\mu}\right]$.

Recall that $z_{1}, \cdots, z_{\mu}$ are distinguished generators of $F_{\mu}$; let us now write $z=\sum z_{i} \pi_{i}$.

Definition 5.1. If $V$ is a module in $\mathcal{S e i}_{\infty}(A)$ let

$$
B(V)=\operatorname{Coker}\left((1-s(1-z)): V\left[F_{\mu}\right] \rightarrow V\left[F_{\mu}\right]\right) .
$$

Since $\epsilon(1-s(1-z))=1$, it is clear that $B(V)$ lies in $\mathcal{F} \mathrm{lk}_{\infty}(A)$. To achieve more symmetric notation we write $z_{i}=y_{i}^{2}$ and deduce $z=y^{2}$ where $y=\sum y_{i} \pi_{i}$. We write $F_{\mu}=F_{\mu}\left(y^{2}\right)$ when we wish to indicate that elements of $F_{\mu}$ are to be written as words in the symbols $y_{i}^{ \pm 2}$. The $A\left[F_{\mu}\right]$-module $\bigoplus_{i=1}^{\mu} \pi_{i} V\left[F_{\mu}\left(y^{2}\right) y_{i}\right]$ is isomorphic to $V\left[F_{\mu}\right]$ and will be written $V\left[F_{\mu}\left(y^{2}\right) y\right]$ for brevity. Now we have

$$
\begin{aligned}
B(V) & \cong \operatorname{Coker}\left(\left(1-s\left(1-y^{2}\right)\right) y^{-1}: V\left[F_{\mu}\left(y^{2}\right) y\right] \rightarrow V\left[F_{\mu}\left(y^{2}\right)\right]\right) \\
& =\operatorname{Coker}\left((1-s) y^{-1}+s y: V\left[F_{\mu}\left(y^{2}\right) y\right] \rightarrow V\left[F_{\mu}\left(y^{2}\right)\right]\right) .
\end{aligned}
$$

In detail,

$$
(1-s) y^{-1}+s y=\sum_{i=1}^{\mu}(1-s) \pi_{i} y_{i}^{-1}+s \pi_{i} y_{i}: v w y_{i} \mapsto(1-s)(v) w+s(v) w y_{i}^{2}
$$


for $v \in \pi_{i}(V), w \in F_{\mu}\left(y^{2}\right)$ and $i \in\{1, \cdots, \mu\}$. A morphism $f: V \rightarrow V^{\prime}$ induces a commutative diagram

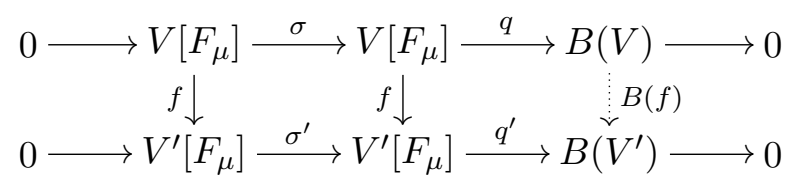

where $\sigma=1-s(1-z)$ and hence induces an $A\left[F_{\mu}\right]$-module map $B(f)$ as shown.

Lemma 5.2. The functor $B$ is exact. In other words, if $V \rightarrow V^{\prime} \rightarrow V^{\prime \prime}$ is an exact sequence in $\mathcal{S} \mathrm{ei}_{\infty}(A)$ then the sequence $B(V) \rightarrow B\left(V^{\prime}\right) \rightarrow B\left(V^{\prime \prime}\right)$ induced is also exact.

Proof. It suffices to show that $B$ preserves short exact sequences. Suppose $0 \rightarrow V \rightarrow V^{\prime} \rightarrow V^{\prime \prime} \rightarrow 0$ is exact. There is a commutative diagram

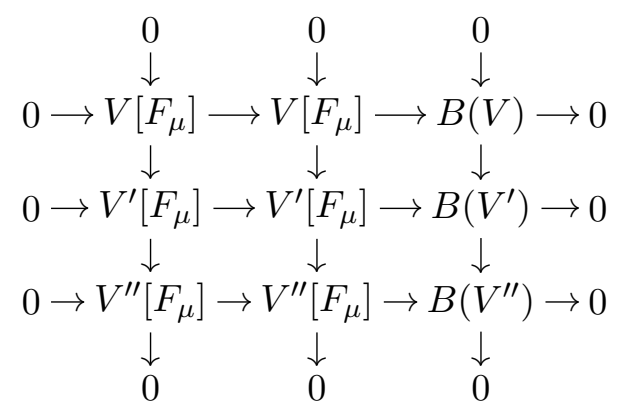

in which the rows and the two left-most columns are exact. It follows that the right-hand column is also exact.

The category $\mathcal{S e i}_{\infty}(A)$ has limits and colimits. For example, the coproduct of a system of modules is the direct sum. Since $B$ is exact and respects arbitrary direct sums $B$ respects all colimits:

Lemma 5.3. The functor $B$ is cocontinuous.

In particular if $V$ is a direct limit $V=\lim V_{i}$ then $B(V)=\lim B\left(V_{i}\right)$. On the other hand $B$ does not respect infinite limits. For example one finds $B\left(\prod V_{i}\right) \not \prod\left(B\left(V_{i}\right)\right)$ because $\left(\prod V_{i}\right)\left[F_{\mu}\right] \varsubsetneqq \prod\left(V_{i}\left[F_{\mu}\right]\right)$. However, $B$ does respect finite limits.

The idea behind the proof of the following proposition is due to A.Ranicki.

Proposition 5.4. The functor $B$ extends to a duality-preserving functor $(B, \Phi,-1): \mathcal{S}$ ei $(A) \rightarrow \mathcal{F} \operatorname{lk}(A)$. 
Proof. For each finitely generated projective $A$-module $V$ there is a natural isomorphism $\Pi_{V}: V^{*}\left[F_{\mu}\right] \rightarrow\left(V\left[F_{\mu}\right]\right)^{*}$ by Example 20. Naturality asserts that for each morphism $\alpha: V \rightarrow W$ the diagram

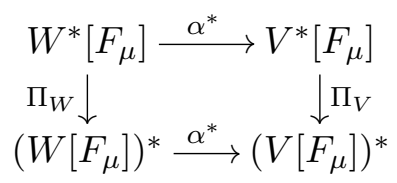

is commutative. Moreover one can check commutativity of

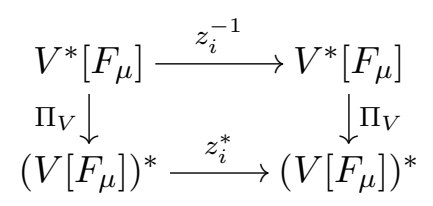

where, as usual, $z_{i}: V\left[F_{\mu}\right] \rightarrow V\left[F_{\mu}\right]$ and $z_{i}: V^{*}\left[F_{\mu}\right] \rightarrow V^{*}\left[F_{\mu}\right]$ denote multiplication on the right by $z_{i}$.

Now if $V \in \mathcal{S}$ ei $(A)$ then there is a commutative diagram:

$$
\begin{aligned}
& 0 \longrightarrow V^{*}\left[F_{\mu}\right] \stackrel{\sigma\left(V^{*}\right)}{\longrightarrow} V^{*}\left[F_{\mu}\right] \longrightarrow B\left(V^{*}\right) \longrightarrow 0
\end{aligned}
$$

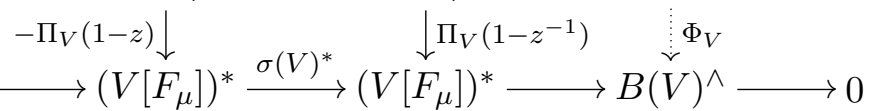

where $\sigma\left(V^{*}\right)=1-\left(1-s^{*}\right)(1-z)$ and $\sigma(V)^{*}=(1-s(1-z))^{*}$. By definition, $\Phi_{V}: B\left(V^{*}\right) \rightarrow B(V)^{\wedge}$ is the induced morphism. Plainly $\Phi_{V}$ is a natural transformation.

The duality-preserving functor $\Pi$ has the property

$$
\Pi_{V}^{*} i_{V\left[F_{\mu}\right]}=\Pi_{V^{*}} i_{V}: V\left[F_{\mu}\right] \rightarrow\left(V^{*}\left[F_{\mu}\right]\right)^{*}
$$

(indeed, this equation features in Definition 2.24). The equations

$$
\begin{aligned}
& \left(\Pi_{V}\left(1-z^{-1}\right)\right)^{*} i_{V\left[F_{\mu}\right]}=\Pi_{V^{*}}(1-z) i_{V} \\
& \left(\Pi_{V}(1-z)\right)^{*} i_{V\left[F_{\mu}\right]}=\Pi_{V^{*}}\left(1-z^{-1}\right) i_{V}
\end{aligned}
$$

imply that

$$
\Phi_{V}^{\wedge} i_{B(V)}=-\Phi_{V^{*}} B\left(i_{V}\right) .
$$

To show that $(B, \Phi,-1)$ is a duality-preserving functor it remains to check that $\Phi$ is an isomorphism. There is a commutative diagram

$$
\begin{gathered}
0 \longrightarrow\left(V\left[F_{\mu}\right]\right)^{*} \stackrel{\sigma(V)^{*}}{\longrightarrow}\left(V\left[F_{\mu}\right]\right)^{*} \longrightarrow B(V)^{\wedge} \longrightarrow 0 \\
\stackrel{\left(1-s^{*}\right) \Pi_{V}^{-1} \downarrow}{\longrightarrow} V^{*}\left[F_{\mu}\right] \stackrel{\sigma\left(V^{*}\right)}{\longrightarrow} V^{*}\left[F_{\mu}\right] \longrightarrow \\
0 \longrightarrow \\
\longrightarrow
\end{gathered}
$$


and the composite morphisms of chain complexes

$$
\begin{gathered}
V^{*}\left[F_{\mu}\right] \stackrel{\sigma\left(V^{*}\right)}{\longrightarrow} V^{*}\left[F_{\mu}\right] \\
\left(1-s^{*}\right)(1-z) \downarrow \\
V^{*}\left[F_{\mu}\right] \underset{\sigma\left(V^{*}\right)}{\longrightarrow} V^{*}\left[F_{\mu}\right]
\end{gathered}
$$

and

$$
\begin{gathered}
\left(V\left[F_{\mu}\right]\right)^{*} \stackrel{\frac{\sigma(V)^{*}}{\longrightarrow}}{\underset{z^{-*}}{ }}\left(V\left[F_{\mu}\right]\right)^{*} \\
\left(1-z^{-*}\right)\left(1-s^{*}\right) \downarrow \\
\left(V\left[F_{\mu}\right]\right)^{*} \underset{\sigma(V)^{*}}{\stackrel{2}{\longrightarrow}}\left(V\left[F_{\mu}\right]\right)^{*}
\end{gathered}
$$

are chain homotopic to the identity by the indicated chain homotopies. [Chain complexes are drawn horizontally and morphisms of chain complexes are given by vertical arrows. The symbol $z^{-*}$ is shorthand for $\left.\left(z^{-1}\right)^{*}\right]$. These composite chain maps therefore induce the identity on $B\left(V^{*}\right)$ and $B(V)^{\wedge}$ respectively so $\Phi_{V}$ is an isomorphism and $(B, \Phi,-1)$ is a duality-preserving functor.

Since $B$ is an exact functor we have:

Corollary 5.5. The functor $(B, \Phi,-1)$ induces a homomorphism of Witt groups

$$
B: W^{\zeta}(\mathcal{S} \operatorname{ei}(A)) \rightarrow W^{-\zeta}(\mathcal{F} \operatorname{lk}(A))
$$

If $(V, \phi)$ is a $\zeta$-hermitian form in $\mathcal{S} \operatorname{ei}(A)$ then the covering construction gives $B(V, \phi)=\left(B(V), \Phi_{V} B(\phi)\right)$ (Lemma 2.29) which can be described explicitly as follows. The morphism $\phi: V \rightarrow V^{*}$ induces $\phi: V\left[F_{\mu}\right] \rightarrow V^{*}\left[F_{\mu}\right]$. Let $\widetilde{\phi}: V\left[F_{\mu}\right] \rightarrow \operatorname{Hom}_{A\left[F_{\mu}\right]_{\Sigma}}\left(V\left[F_{\mu}\right]_{\Sigma}, A\left[F_{\mu}\right]_{\Sigma}\right)$ denote the composition of $\phi$ with

$$
\Pi_{V}: V^{*}\left[F_{\mu}\right] \rightarrow\left(V\left[F_{\mu}\right]\right)^{*}=\operatorname{Hom}_{A\left[F_{\mu}\right]}\left(V\left[F_{\mu}\right], A\left[F_{\mu}\right]\right)
$$

(see Example 2.26) and the localization

$$
\operatorname{Hom}_{A\left[F_{\mu}\right]}\left(V\left[F_{\mu}\right], A\left[F_{\mu}\right]\right) \rightarrow \operatorname{Hom}_{A\left[F_{\mu}\right]_{\Sigma}}\left(V\left[F_{\mu}\right]_{\Sigma}, A\left[F_{\mu}\right]_{\Sigma}\right) .
$$

If $m, m^{\prime} \in B(V)$ we may write $m=q(x), m^{\prime}=q\left(x^{\prime}\right)$ for some $x, x^{\prime} \in V\left[F_{\mu}\right]$. 
It follows from Remark 2.18 that in $\frac{A\left[F_{\mu}\right]_{\Sigma}}{A\left[F_{\mu}\right]}$ we have

$$
\begin{aligned}
\Phi_{V} B(\phi)(m)\left(m^{\prime}\right) & =\Phi_{V} B(\phi)(q(x))\left(q\left(x^{\prime}\right)\right) \\
& =q^{\prime}\left(\Pi_{V}\left(1-z^{-1}\right) \phi(x)\right)\left(q\left(x^{\prime}\right)\right) \quad(\text { using }(34)) \\
& =\left(\operatorname{id} \otimes \Pi_{V}\left(1-z^{-1}\right) \phi(x)\right)(\operatorname{id} \otimes \sigma)^{-1}\left(1 \otimes x^{\prime}\right) \\
& =\widetilde{\phi}(x)\left((1-z)(\operatorname{id} \otimes \sigma)^{-1}\left(1 \otimes x^{\prime}\right)\right) .
\end{aligned}
$$

Remark 5.6. If $V \in \mathcal{S}$ ei $(\mathbb{Z})$ and $\phi: V \rightarrow V^{*}$ is the Seifert form corresponding to a Seifert surface for an $F_{\mu}$-link then by $(35) \Phi_{V} B(\phi): B(V) \rightarrow$ $B(V)^{\wedge}$ is the corresponding Blanchfield-Duval form for the $F_{\mu}$-link; compare Kearton [23], Levine [33, Prop 14.3], Cochran and Orr [7, Thm4.2] and Ranicki [43, Defn32.7]. For example, setting $r=\phi(x), s=\phi\left(x^{\prime}\right), \Gamma=z, \theta=\phi s$, and $\epsilon=\zeta$ one obtains from (35) the equations appearing immediately prior to Theorem 4.2 in [7].

For the proof of Theorem 1.4 in Section 6 we will need the observation that $(B, \Phi,-1)$ respects a change of coefficients from $\mathbb{Z}$ to $\mathbb{Q}$. Let us make a more general statement. Recall from equation (19) the definition of composition for duality-preserving functors.

Proposition 5.7. A ring homomorphism $A \rightarrow A^{\prime}$ induces a diagram of duality-preserving functors

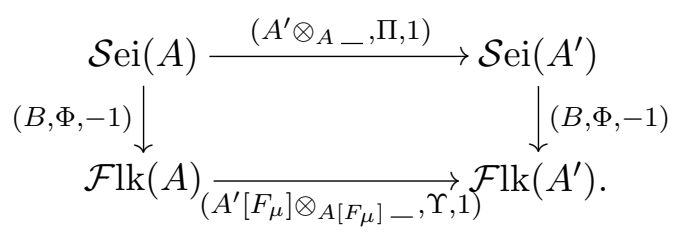

which commutes up to natural isomorphism. Consequently, there is a commutative diagram of Witt groups

$$
\begin{gathered}
W^{\zeta}(\mathcal{S} \operatorname{ei}(A)) \longrightarrow W^{\zeta}\left(\mathcal{S} \operatorname{sei}\left(A^{\prime}\right)\right) \\
B \downarrow \\
\downarrow B \\
W^{-\zeta}(\mathcal{F} \operatorname{lk}(A)) \rightarrow W^{-\zeta}\left(\mathcal{F} \operatorname{lk}\left(A^{\prime}\right)\right) .
\end{gathered}
$$

Proof. See Appendix A. 


\subsection{Adjunction}

We leave duality structures behind for the present and prove that the functor $B: \mathcal{S} \operatorname{ei}_{\infty}(A) \rightarrow \mathcal{F} \mathrm{lk}_{\infty}(A)$ is left adjoint to $U: \mathcal{F} \mathrm{lk}_{\infty}(A) \rightarrow \mathcal{S} \mathrm{ei}_{\infty}(A)$.

Definition 5.8. Suppose $F: \mathcal{C} \rightarrow \mathcal{D}$ is a functor. A functor $G: \mathcal{D} \rightarrow \mathcal{C}$ is called left adjoint to $F$ if there exists a natural transformation $\theta: \operatorname{id}_{\mathcal{D}} \rightarrow F G$ such that for every object $D \in \mathcal{D}$ the morphism $\theta_{D}: D \rightarrow F G(D)$ has the following universal property: For every morphism $d$ in $\mathcal{D}$ of the form $d: D \rightarrow F(C)$ there is a unique morphism $c: G(D) \rightarrow C$ in $\mathcal{C}$ such that $d=F(c) \theta_{D}$.

$$
D \underset{\theta_{D}}{\underset{F G(D)}{F(c)}} F(C)
$$

Let us recall a few examples: 1) The inclusion of the category of abelian groups in the category of groups has left adjoint known as "abelianization" which sends a group $G$ to $G /[G, G]$. 2) The inclusion of the category of compact Hausdorff topological spaces in the category of (all) topological spaces has a left adjoint known as "Stone-Čech compactification". 3) Colimit constructions (e.g. direct limit or coproduct) can be expressed via a left adjoint as follows. Suppose $\mathcal{C}$ is a category, $J$ is a small category and $\mathcal{C}^{J}$ denotes the category of functors $J \rightarrow \mathcal{C}$. If there is a left adjoint to the constant functor $\mathcal{C} \rightarrow \mathcal{C}^{J}$ then that left adjoint sends each functor $J \rightarrow \mathcal{C}$ to its colimit in $\mathcal{C}$ (and the colimit exists).

Proposition 5.9. The functor $B: \mathcal{S} \operatorname{ei}_{\infty}(A) \rightarrow \mathcal{F} \mathrm{k}_{\infty}(A)$ is left adjoint to $U: \mathcal{F} \mathrm{lk}_{\infty}(A) \rightarrow \mathcal{S} \operatorname{ei}_{\infty}(A)$.

The required map $\theta_{V}: V \rightarrow U B(V)$ is the restriction of the map $q:$ $V\left[F_{\mu}\right] \rightarrow B(V)$ in the diagram (33) above. In symbols $\theta_{V}=q \mid: V \rightarrow$ $U B(V)$. During the proof of Proposition 5.9 below we show that $\theta_{V}$ is a morphism of Seifert modules. It follows from the diagram (33) that $\theta:$ id $\rightarrow$ $U B$ is a natural transformation.

Before proving Proposition 5.9, we note some consequences:

Corollary 5.10. Let $V \in \mathcal{S} \mathrm{ei}_{\infty}(A)$ and $M \in \mathcal{F} \mathrm{k}_{\infty}(A)$. There is a natural isomorphism $\psi_{M}: B U(M) \rightarrow M$ and the composites

$$
\begin{aligned}
& U(M) \stackrel{\theta_{U(M)}}{\longrightarrow} U B U(M) \stackrel{U\left(\psi_{M}\right)}{\longrightarrow} U(M) \\
& B(V) \stackrel{B\left(\theta_{V}\right)}{\longrightarrow} B U B(V) \stackrel{\psi_{B(V)}}{\longrightarrow} B(V)
\end{aligned}
$$

are identity morphisms. In particular $\theta_{U(M)}$ and $B\left(\theta_{V}\right)$ are isomorphisms. 
The existence of a natural transformation $\psi_{M}: B U(M) \rightarrow M$ follows from Proposition 5.9 alone. To prove that $\psi$ is an isomorphism one requires the additional information that $U$ is full and faithful. We are not claiming that $\theta_{V}: V \rightarrow U B(V)$ is an isomorphism. Indeed, $U$ and $B$ are not equivalences of categories.

Proof of Corollary 5.10. Let $\psi_{M}: B U(M) \rightarrow M$ be the unique morphism such that $\operatorname{id}_{U(M)}=U\left(\psi_{M}\right) \theta_{U(M)}$. One can check that $\psi_{M}$ is a natural transformation and that $\psi_{B(V)} B\left(\theta_{V}\right)=\mathrm{id}_{B(V)}$ (see for example Theorem 3.1 .5 of [4]). The functor $U: \mathcal{F} \operatorname{lk}_{\infty}(A) \rightarrow \mathcal{S e i}_{\infty}(A)$ is full and faithful by Lemma 4.2 so $\psi_{M}$ is an isomorphism (see Theorem 3.4.1 of [4]). It follows that $\theta_{U(V)}$ and $B\left(\theta_{V}\right)$ are isomorphisms.

Proof of Proposition 5.9. By Definition 5.8 there are two statements to prove:

1. The map $\theta_{V}: V \rightarrow U B(V)$ is a morphism of Seifert modules.

2. If $M \in \mathcal{F} \operatorname{lk}_{\infty}(A)$ and $f: V \rightarrow U(M)$ is a morphism in $\mathcal{S e i}_{\infty}(A)$ then there is a unique morphism $g: B(V) \rightarrow M$ such that $f=U(g) \theta_{V}$.

As we remarked above, it follows from the diagram (33) that $\theta:$ id $\rightarrow U B$ is a natural transformation. We shall need the following lemma which is proved shortly below:

Lemma 5.11. Suppose $V \in \mathcal{S}_{\operatorname{ei}_{\infty}}(A), M \in \mathcal{F} \mathrm{k}_{\infty}(A)$ and $f: V \rightarrow M$ is an $A$-module morphism. Let $\widetilde{f}: V\left[F_{\mu}\right] \rightarrow M$ denote the induced $A\left[F_{\mu}\right]$-module morphism. The map $f: V \rightarrow U(M)$ is a morphism in $\mathcal{S e i}_{\infty}(A)$ if and only if $f(x)=\widetilde{f}(s(1-z) x)$ for all $x \in V$.

Let us deduce statement 1. above. By the definition of $B(V)$ there is an exact sequence

$$
0 \rightarrow V\left[F_{\mu}\right] \stackrel{1-s(1-z)}{\longrightarrow} V\left[F_{\mu}\right] \stackrel{q}{\rightarrow} B(V) \rightarrow 0
$$

so $q(x)=q(s(1-z) x)$ for all $x \in V$. By Lemma 5.11, $\theta_{V}=\left.q\right|_{V}$ is a morphism of Seifert modules.

We turn now to statement 2. Since $V$ generates $B(V)$ as an $A\left[F_{\mu}\right]$ module, an $A\left[F_{\mu}\right]$-module morphism $g: B(V) \rightarrow M$ satisfies $f=U(g) \theta_{V}$ if and only if $g$ fits into the diagram

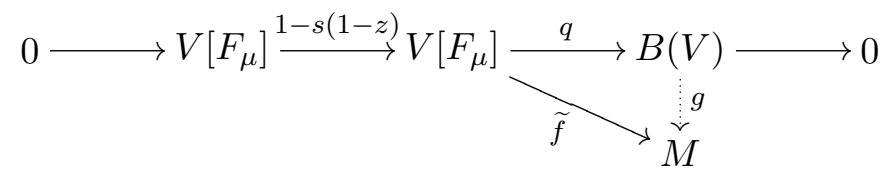


Since $f$ is a morphism of Seifert modules we have $f(x)=\widetilde{f}(s(1-z) x)$ for all $x \in V$ by Lemma 5.11. So $\widetilde{f} \sigma=0$, and therefore there exists unique $g: B(V) \rightarrow M$ such that $g q=\tilde{f}$. It follows that there exists unique $g$ such that $f=U(g) \theta_{V}$. Thus we have established both 1. and 2. assuming Lemma 5.11.

Proof of Lemma 5.11. The Seifert module structure on $U(M)$ is given by (30) above so $f$ is a Seifert morphism if and only if

a) $\omega \gamma^{-1} f(x)=f(s x)$ and

b) $\gamma p_{i} \gamma^{-1} f(x)=f\left(\pi_{i} x\right)$ for each $x \in V$.

To prove the 'if' part of Lemma 5.11, suppose $f(x)=\widetilde{f}(s(1-z) x)$.

a) The equations $\omega \gamma^{-1}\left(\sum_{i=1}^{\mu}\left(1-z_{i}\right) x_{i}\right)=\sum_{i=1}^{\mu} x_{i}$ and

$$
f(x)=\widetilde{f}(s(1-z) x)=\sum_{i=1}^{\mu} \widetilde{f}\left(s\left(1-z_{i}\right) \pi_{i} x\right)=\sum_{i=1}^{\mu}\left(1-z_{i}\right) f\left(s \pi_{i} x\right) .
$$

imply that $\omega \gamma^{-1} f(x)=\omega \gamma^{-1} \sum_{i=1}^{\mu}\left(1-z_{i}\right) f\left(s \pi_{i} x\right)=\sum_{i=1}^{\mu} f\left(s \pi_{i} x\right)=f(s x)$.

b) Observe that

$$
f\left(\pi_{i} x\right)=\widetilde{f}\left(s(1-z) \pi_{i} x\right)=\sum_{j=1}^{\mu} \widetilde{f}\left(s\left(1-z_{j}\right) \pi_{j} \pi_{i} x\right)=\left(1-z_{i}\right) f\left(s \pi_{i} x\right) .
$$

while

$$
\begin{aligned}
\gamma p_{i} \gamma^{-1} f(x)=\gamma p_{i} \gamma^{-1} \widetilde{f}(s(1-z) x) & =\gamma p_{i} \gamma^{-1} \sum_{j=1}^{\mu}\left(1-z_{j}\right) f\left(s \pi_{j} x\right) \\
& =\left(1-z_{i}\right) f\left(s \pi_{i} x\right) .
\end{aligned}
$$

Thus $f\left(\pi_{i} x\right)=\gamma p_{i} \gamma^{-1} f(x)$.

To prove the "only if" part of Lemma 5.11, suppose we have a) and b) 
above. Now

$$
\begin{aligned}
\widetilde{f}(s(1-z) x) & =\sum_{i=1}^{\mu}\left(1-z_{i}\right) f\left(s \pi_{i} x\right) \\
& =\sum_{i=1}^{\mu}\left(1-z_{i}\right)\left(\omega \gamma^{-1}\right)\left(\gamma p_{i} \gamma^{-1}\right) f(x) \\
& =\sum_{i=1}^{\mu}\left(1-z_{i}\right) \omega p_{i} \gamma^{-1} f(x) \\
& =f(x) .
\end{aligned}
$$

This completes the proof of Proposition 5.9.

\subsection{Localization}

When one passes from Seifert modules to $F_{\mu}$-link modules, certain Seifert modules disappear altogether; following Farber we shall call such modules primitive.

Definition 5.12. Let $\operatorname{Prim}_{\infty}(A)$ denote the full subcategory of $\mathcal{S} \mathrm{ei}_{\infty}(A)$ containing precisely the modules $V$ such that $B(V)=0$. Modules in $\operatorname{Prim}_{\infty}(A)$ will be called primitive.

For example, if $V$ is a Seifert module with $\rho: P_{\mu} \rightarrow \operatorname{End}_{A} V$ such that $\rho(s)=0$ or $\rho(s)=1$ then

$$
(1-\rho(s)) y^{-1}+\rho(s) y: V\left[F_{\mu}\left(y^{2}\right) y\right] \rightarrow V\left[F_{\mu}\left(y^{2}\right)\right]
$$

is an isomorphism and therefore has zero cokernel. A module in $\mathcal{S e i}_{\infty}(A)$ with $\rho(s)=0$ or 1 will be called trivially primitive. We show in Section 5.5 that all the primitive Seifert modules in $\mathcal{S e i}_{\infty}(A)$ can be "built" from trivially primitive modules. If $A$ is semi-simple Artinian then a similar result applies when one restricts attention to the category $\mathcal{S}$ ei $(A)$ of representations of $P_{\mu}$ by finitely generated projective $A$-modules: Every primitive in $\mathcal{S}$ ei $(A)$ can be "built" from a finite number of trivially primitive modules in $\mathcal{S}$ ei $(A)$ (see Proposition 5.33). This statement is not true for all rings $A$; one must consider primitives which exhibit a kind of nilpotence. Such primitives were described by Bass, Heller and Swan when $\mu=1$ (see also Ranicki [44]). The general case $\mu \geq 1$ will be analyzed in a subsequent paper (joint work with A.Ranicki) [46]. 
In the present section we construct an equivalence between $\mathcal{F} \mathrm{lk}_{\infty}(A)$ and a quotient category $\mathcal{S e i}_{\infty}(A) / \mathcal{P r i m}_{\infty}(A)$. This quotient is an example of universal localization for categories; the objects in $\mathcal{S e i}_{\infty}(A) / \mathcal{P r i m}_{\infty}(A)$ are the same as the objects in $\mathcal{S e i}_{\infty}(A)$ but the morphisms are different. The universal property is that a morphism in $\mathcal{S e i}_{\infty}(A)$ whose kernel and cokernel are in $\mathcal{P r i m}_{\infty}(A)$ has an inverse in $\mathcal{S e i}_{\infty}(A) / \mathcal{P} \operatorname{rim}_{\infty}(A)$. A more detailed construction of the quotient appears in Section 5.6. We proceed to derive an equivalence between $\mathcal{F} \operatorname{lk}(A)$ and a corresponding quotient of $\mathcal{S} \operatorname{ei}(A)$.

Definition 5.13. The functor $F: \mathcal{S e i}_{\infty}(A) \rightarrow \mathcal{S e i}_{\infty}(A) / \mathcal{P} \operatorname{rim}_{\infty}(A)$ is the universal functor which makes invertible all morphisms whose kernel and cokernel are primitive. In other words, any functor which makes these morphisms invertible factors uniquely through $F$.

We outline in Section 5.6 one construction of $F$ which will be convenient for our purposes; see Gabriel [20] or Srinivas [53, Appendix B.3] for further details. A more general construction can be found in Gabriel and Zisman [21] or Borceux [4, Ch5]. It follows directly from the definition that the localization $F$ is unique (up to unique isomorphism).

Applying Definition 5.13 to the functor $B: \mathcal{S} \mathrm{ei}_{\infty}(A) \rightarrow \mathcal{F} \mathrm{lk}_{\infty}(A)$, there is a unique functor $\bar{B}$ such that $B=\bar{B} F$ :

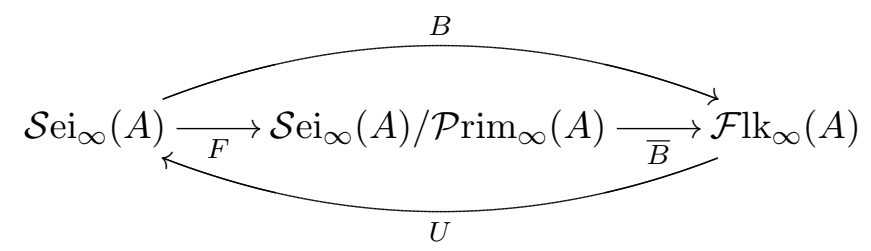

Proposition 5.9 stated that $B: \mathcal{S e i}_{\infty}(A) \rightarrow \mathcal{F} \mathrm{k}_{\infty}(A)$ is left adjoint to the forgetful functor $U$. We deduce in the next proposition that $B$ satisfies the same universal property as $F$, but only "up to natural isomorphism". If $f$ is a morphism in $\mathcal{S e i}_{\infty}(A)$ let us write $f \in \Xi_{\infty}$ if the kernel and cokernel of $f$ both lie in $\mathcal{P r i m}_{\infty}(A)$.

Proposition 5.14. If $G: \mathcal{S e i}_{\infty}(A) \rightarrow \mathcal{B}$ is a functor which sends every morphism in $\Xi_{\infty}$ to an invertible morphism then there is a functor

$$
\widetilde{G}: \mathcal{F} \mathrm{lk}_{\infty}(A) \rightarrow \mathcal{B}
$$

such that $\widetilde{G} B$ is naturally isomorphic to $G$. The functor $\widetilde{G}$ is unique up to natural isomorphism. 
Proof. We prove uniqueness first. If there is a natural isomorphism $G \simeq \widetilde{G} B$ then $G U \simeq \widetilde{G} B U \simeq \widetilde{G}$ by Corollary 5.10 .

To prove existence we must show that if $\widetilde{G}=G U$ then $\widetilde{G} B \simeq G$. Indeed, by Corollary $5.10 B\left(\theta_{V}\right): B(V) \rightarrow B U B(V)$ is an isomorphism for each $V \in \mathcal{S e i}_{\infty}(A)$. Since $B$ respects exact sequences we have $\theta_{V} \in \Xi_{\infty}$. It follows that $G(\theta): G \rightarrow G U B=\widetilde{G} B$ is a natural isomorphism.

The following is an immediate consequence of the fact that $F$ and $B$ have the same universal property (up to natural isomorphism):

Corollary 5.15. The functor $\bar{B}: \mathcal{S e i}_{\infty}(A) / \mathcal{P} \operatorname{rim}_{\infty}(A) \rightarrow \mathcal{F} \mathrm{k}_{\infty}(A)$ is an equivalence.

We turn now to the categories $\mathcal{S} \operatorname{ei}(A)$ and $\mathcal{F} \operatorname{lk}(A)$.

Definition 5.16. Let $\mathcal{S}$ ei $(A) / \mathcal{P}_{\operatorname{rim}_{\infty}}(A) \subset \mathcal{S} \operatorname{ei}_{\infty}(A) / \mathcal{P r i m}_{\infty}(A)$ denote the full subcategory whose objects are precisely the modules in $\mathcal{S}$ ei $(A)$ (i.e. the modules which are finitely generated and projective as $A$-modules).

There is a commutative diagram of functors

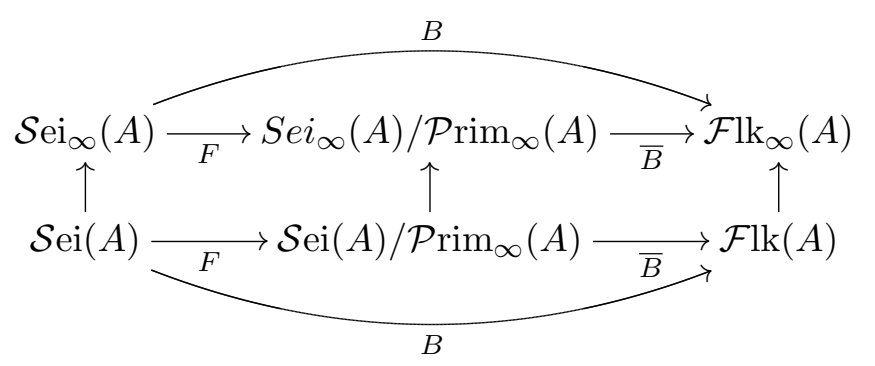

in which all the vertical arrows are inclusions of full subcategories.

Theorem 5.17. The functor $\bar{B}: \mathcal{S} \operatorname{ei}(A) / \mathcal{P} \operatorname{mim}_{\infty}(A) \rightarrow \mathcal{F} \operatorname{lk}(A)$ is an equivalence of categories.

We will use the following general lemma in the proof of Theorem 5.17. Recall that a functor $G: \mathcal{C} \rightarrow \mathcal{D}$ is called full and faithful if it induces an isomorphism $\operatorname{Hom}_{\mathcal{C}}\left(V, V^{\prime}\right) \rightarrow \operatorname{Hom}_{\mathcal{D}}\left(G(V), G\left(V^{\prime}\right)\right)$ for every pair of objects $V, V^{\prime} \in \mathcal{C}$.

Lemma 5.18. A functor $G: \mathcal{C} \rightarrow \mathcal{D}$ is an equivalence of categories if and only if $G$ is full and faithful and every object in $\mathcal{D}$ is isomorphic to $G(V)$ for some $V \in \mathcal{C}$.

Proof. See for example Borceux [4, Prop 3.4.3]. 
It follows from Corollary 5.15 and Lemma 5.18 that

$$
\bar{B}: \mathcal{S} \operatorname{ei}(A) / \mathcal{P} \operatorname{rim}_{\infty}(A) \rightarrow \mathcal{F} \operatorname{lk}(A)
$$

is full and faithful. Theorem 5.17 is therefore a consequence of the following proposition:

Proposition 5.19. Every module in $\mathcal{F} \operatorname{lk}(A)$ is isomorphic to $B(V)$ for some $V \in \mathcal{S}$ ei $(A)$.

Proof. By definition, every module $M \in \mathcal{F} \operatorname{lk}(A)$ has presentation

$$
0 \rightarrow V\left[F_{\mu}\right] \stackrel{\sigma}{\rightarrow} V\left[F_{\mu}\right] \rightarrow M \rightarrow 0
$$

where $V$ is a finitely generated projective $A$-module and $\epsilon(\sigma): V \rightarrow V$ is an isomorphism. Given any $A$-module $W$ there is a canonical isomorphism

$$
\operatorname{Hom}_{A\left[F_{\mu}\right]}\left(V\left[F_{\mu}\right], W\left[F_{\mu}\right]\right) \cong \operatorname{Hom}_{A}(V, W)\left[F_{\mu}\right]
$$

and in particular $\sigma$ can be expressed uniquely as a sum $\sum_{w \in F_{\mu}} \sigma_{w} w$ with each $\sigma_{w} \in \operatorname{Hom}_{A}(V, V)$.

Lemma 5.20. Every $M \in \mathcal{F} \operatorname{lk}(A)$ is isomorphic to the cokernel of an endomorphism $\sigma: V\left[F_{\mu}\right] \rightarrow V\left[F_{\mu}\right]$ of the form

$$
\sigma=1+\sigma_{1}\left(1-z_{1}\right)+\cdots+\sigma_{\mu}\left(1-z_{\mu}\right)
$$

where $V$ is finitely generated and projective and $\sigma_{1}, \cdots, \sigma_{\mu} \in \operatorname{Hom}_{A}(V, V)$.

Proof of Lemma. By the definition of $\mathcal{F} \operatorname{lk}(A)$, the module $M$ is isomorphic to the cokernel of some map $\sigma: V\left[F_{\mu}\right] \rightarrow V\left[F_{\mu}\right]$ where $V$ is finitely generated and projective. The idea of this proof is to reduce the support of $\sigma$ to $\left\{1, z_{1}, \cdots, z_{\mu}\right\} \subset F_{\mu}$ at the expense of replacing $V$ by a larger finitely generated projective module. Note first that

$$
\operatorname{Coker}(\sigma) \cong \operatorname{Coker}\left(\begin{array}{cc}
\sigma & 0 \\
0 & 1
\end{array}\right):\left(V \oplus V^{\prime}\right)\left[F_{\mu}\right] \rightarrow\left(V \oplus V^{\prime}\right)\left[F_{\mu}\right]
$$

where $V^{\prime}$ is any $A$-module and 1 denotes the identity morphism. The equation

$$
\left(\begin{array}{cc}
1 & -b \\
0 & 1
\end{array}\right)\left(\begin{array}{cc}
a+b c & 0 \\
0 & 1
\end{array}\right)\left(\begin{array}{ll}
1 & 0 \\
c & 1
\end{array}\right)=\left(\begin{array}{cc}
a & -b \\
c & 1
\end{array}\right)
$$


therefore implies that Coker $\left(\begin{array}{cc}a & -b \\ c & 1\end{array}\right)$ is isomorphic to Coker $(a+b c)$. Repeated application of equation (39) implies that $M$ is isomorphic to the cokernel of an endomorphism $\sigma=\sigma_{0}+\sum_{i=1}^{\mu} \sigma_{i}^{+} z_{i}+\sum_{i=1}^{\mu} \sigma_{i}^{-} z_{i}^{-1}$ with $\sigma_{0}, \sigma_{i}^{+}$ and $\sigma_{i}^{-}$in $\operatorname{Hom}_{A}(V, V)$ for some $V$. For each of the indices $i=1, \cdots, \mu$ in turn, one can apply the identity $\operatorname{Coker}(\sigma)=\operatorname{Coker}\left(\sigma z_{i}\right)$ followed by further equations (39). One obtains an identity $M \cong \operatorname{Coker}(\beta)$ where $\beta=\beta_{0}+\beta_{1} z_{1}+\cdots+\beta_{\mu} z_{\mu}$ and $\beta_{i} \in \operatorname{Hom}_{A}(V, V)$ for some finitely generated projective module $V$ over $A$. By Lemma 2.3, $\epsilon(\beta)$ is an isomorphism. Let $\sigma=\epsilon(\beta)^{-1} \beta$. Now $\epsilon(\sigma)=1$ and so

$$
\sigma=1+\sigma_{1}\left(z_{1}-1\right)+\cdots \sigma_{\mu}\left(z_{\mu}-1\right)
$$

for some $\sigma_{1}, \cdots, \sigma_{\mu} \in \operatorname{Hom}_{A}(V, V)$. This completes the proof of Lemma 5.20.

We may now finish the proof of Proposition 5.19. If

$$
\sigma=1+\sum_{i} \sigma_{i}\left(1-z_{i}\right)
$$

then the equation

$$
\begin{aligned}
& \left(\begin{array}{cccc}
1 & 0 & \cdots & 0 \\
1 & 1 & \cdots & 0 \\
\vdots & \vdots & \ddots & \vdots \\
1 & 0 & \cdots & 1
\end{array}\right)\left(\begin{array}{cccc}
1 & \sigma_{2}\left(1-z_{2}\right) & \cdots & \sigma_{\mu}\left(1-z_{\mu}\right) \\
0 & 1 & \cdots & 0 \\
\vdots & \vdots & \ddots & \vdots \\
0 & 0 & \cdots & 1
\end{array}\right)\left(\begin{array}{cccc}
\sigma & 0 & \cdots & 0 \\
0 & 1 & \cdots & 0 \\
\vdots & \vdots & \ddots & \vdots \\
0 & 0 & \cdots & 1
\end{array}\right)\left(\begin{array}{cccc}
1 & 0 & \cdots & 0 \\
-1 & 1 & \cdots & 0 \\
\vdots & \vdots & \ddots & \vdots \\
-1 & 0 & \cdots & 1
\end{array}\right) \\
& =\left(\begin{array}{cccc}
1+\sigma_{1}\left(1-z_{1}\right) & \sigma_{2}\left(1-z_{2}\right) & \cdots & \sigma_{\mu}\left(1-z_{\mu}\right) \\
\sigma_{1}\left(1-z_{1}\right) & 1+\sigma_{2}\left(1-z_{2}\right) & \cdots & \sigma_{\mu}\left(1-z_{\mu}\right) \\
\vdots & \vdots & \ddots & \vdots \\
\sigma_{1}\left(1-z_{1}\right) & \sigma_{2}\left(1-z_{2}\right) & \cdots & 1+\sigma_{\mu}\left(1-z_{\mu}\right)
\end{array}\right)
\end{aligned}
$$

implies that

$$
\operatorname{Coker}(\sigma) \cong \operatorname{Coker}\left(1-s(1-z): V^{\oplus \mu}\left[F_{\mu}\right] \rightarrow V^{\oplus \mu}\left[F_{\mu}\right]\right)
$$

where $\pi_{i}$ acts as projection on the $i$ th component of $V^{\oplus \mu}$ and $s$ acts as

$$
\left(\begin{array}{cccc}
\sigma_{1} & \sigma_{2} & \cdots & \sigma_{\mu} \\
\sigma_{1} & \sigma_{2} & \cdots & \sigma_{\mu} \\
\vdots & \vdots & \ddots & \vdots \\
\sigma_{1} & \sigma_{2} & \cdots & \sigma_{\mu}
\end{array}\right)
$$

Thus $M \cong B\left(V^{\oplus \mu}\right)$.

This completes the proof of Theorem 5.17. 


\subsection{Duality in the quotient}

Having established that $\bar{B}: \mathcal{S} \operatorname{ei}(A) / \mathcal{P} \operatorname{rim}_{\infty}(A) \rightarrow \mathcal{F l k}(A)$ is an equivalence, we may use $\bar{B}$ to give duality structure to $\mathcal{S} \operatorname{ei}(A) / \mathcal{P} \operatorname{rim}_{\infty}(A)$ and make the lower part of (38) a commutative diagram of duality-preserving functors. Since the objects in $\mathcal{S}$ ei $(A) / \mathcal{P} \operatorname{rim}_{\infty}(A)$ coincide with those in $\mathcal{S}$ ei $(A)$ we define $F(V)^{*}=F\left(V^{*}\right)$ and $i_{F(V)}=F\left(i_{V}\right): F(V) \rightarrow F(V)^{* *}$ where

$$
F: \mathcal{S} \operatorname{ei}(A) \rightarrow \mathcal{S} \operatorname{ei}(A) / \mathcal{P} \operatorname{rim}_{\infty}(A)
$$

is the canonical functor. If $f: V \rightarrow V^{\prime}$ is a morphism in $\mathcal{S}$ ei $(A) / \mathcal{P r i m}_{\infty}(A)$ let

$$
f^{*}=\bar{B}^{-1}\left(\Phi_{V}^{-1} \bar{B}(f)^{\wedge} \Phi_{V^{\prime}}\right): V^{\prime *} \rightarrow V^{*} .
$$

It is easy to see that ${ }^{*}$ is a contravariant functor and that $i_{V}^{*} i_{V^{*}}=i d_{V^{*}}$ for all $V$ so $\mathcal{S}$ ei $(A) / \mathcal{P r i m}_{\infty}(A)$ is a hermitian category.

Recall that the composite of duality-preserving functors is defined by

$$
(G, \Psi, \eta) \circ\left(G^{\prime}, \Psi^{\prime}, \eta^{\prime}\right)=\left(G G^{\prime}, \Psi G\left(\Psi^{\prime}\right), \eta \eta^{\prime}\right) .
$$

Proposition 5.21. The duality-preserving functor

$$
(B, \Phi,-1): \mathcal{S} \operatorname{ei}(A) \rightarrow \mathcal{F} \operatorname{lk}(A)
$$

coincides with the composite $(\bar{B}, \Phi,-1) \circ(F, \mathrm{id}, 1)$.

Proof. It follows from equation (40) and Proposition 5.4 that $(\bar{B}, \Phi,-1)$ is a duality-preserving functor.

By definition $F(V)^{*}=F\left(V^{*}\right)$ and $i_{F(V)}=F\left(i_{V}\right)$; to show that

$$
(F, \mathrm{id}, 1): \mathcal{S} \operatorname{ei}(A) \rightarrow \mathcal{S} \operatorname{ei}(A) / \mathcal{P} \operatorname{rim}_{\infty}(A)
$$

is a duality-preserving functor we must check that $F(f)^{*}=F\left(f^{*}\right)$ for each morphism $f: V \rightarrow V^{\prime}$ in $\mathcal{S}$ ei $(A)$. Indeed,

$$
\begin{aligned}
F(f)^{*} & =\bar{B}^{-1}\left(\Phi_{V}^{-1} \bar{B} F(f)^{\wedge} \Phi_{V^{\prime}}\right) \\
& =\bar{B}^{-1}\left(\Phi_{V}^{-1} B(f)^{\wedge} \Phi_{V^{\prime}}\right) \\
& =\bar{B}^{-1}\left(B\left(f^{*}\right)\right) \quad(\text { since } \Phi \text { is natural }) \\
& =F\left(f^{*}\right) .
\end{aligned}
$$

It is easy to verify that $(B, \Phi,-1)=(\bar{B}, \Phi,-1) \circ(F$, id, 1$)$. 
Proposition 5.22. Let $\zeta=1$ or -1 . The duality-preserving functor

$$
(\bar{B}, \Phi,-1): \mathcal{S} \mathrm{ei}(A) / \mathcal{P} \operatorname{rim}_{\infty}(A) \rightarrow \mathcal{F} \operatorname{lk}(A)
$$

is an equivalence of hermitian categories and induces an isomorphism of Witt groups

$$
\bar{B}: W^{\zeta}\left(\mathcal{S} \operatorname{ei}(A) / \mathcal{P} \operatorname{rim}_{\infty}(A)\right) \rightarrow W^{-\zeta}(\mathcal{F} \operatorname{lk}(A)) .
$$

Proof. Since $\bar{B}$ is an equivalence of categories (by Theorem 5.17 above) it follows that $(\bar{B}, \Phi,-1)$ is an equivalence of hermitian categories (see Proposition II.7 of [51]). It also follows that $\bar{B}$ preserves limits and colimits so $\bar{B}$ preserves exact sequences and hence induces a homomorphism (41) of Witt groups. By Lemma A.2 of Appendix A this homomorphism is an isomorphism (41).

\subsection{Structure of Primitives}

Recall that a module $(V, \rho)$ in $\mathcal{S} \operatorname{ei}_{\infty}(A)$ is called trivially primitive if $\rho(s)=0$ or $\rho(s)=1$. In this section we prove that every primitive module in $\mathcal{S e i}_{\infty}(A)$ is composed of trivially primitive modules.

Lemma 5.23. If $(V, \rho) \in \mathcal{S}_{\mathrm{ei}}^{\infty}(A)$ and there exists a non-zero element $x \in V$ such that $\rho\left(s \pi_{i}\right) x=0$ for all $i$ then $V$ has a non-zero submodule $\left(V^{\prime}, \rho^{\prime}\right)$ such that $\rho^{\prime}(s)=0$.

Proof. Note that $x=\sum \pi_{i} x$ and at least one of the terms $\pi_{i} x$ must be non-zero. Choose non-zero $V^{\prime}=A \pi_{i} x$ and define $\rho^{\prime}$ by

$$
\rho^{\prime}\left(\pi_{j}\right)=\left\{\begin{array}{l}
1 \text { if } j=i \\
0 \text { if } j \neq i
\end{array} \quad, \quad \rho^{\prime}(s)=0 .\right.
$$

Now $\left(V^{\prime}, \rho^{\prime}\right)$ is the required non-zero submodule of $(V, \rho)$.

Lemma 5.24. If $(V, \rho)$ is primitive and non-zero then there exists a nonzero trivially primitive submodule $\left(V^{\prime}, \rho^{\prime}\right)$.

Proof. (compare Lemma 7.10c in Farber [17]) Since $(V, \rho)$ is primitive,

$$
\rho(1-s) y^{-1}+\rho(s) y: V\left[F_{\mu}\left(y^{2}\right) y\right] \rightarrow V\left[F_{\mu}\left(y^{2}\right)\right]
$$

is an isomorphism with inverse $\alpha$ say. Now $\alpha$ can be written as a finite sum $\sum_{w \in S} \alpha_{w} w$ where $S$ is a finite subset of $\bigcup_{i=1}^{\mu} F_{\mu}\left(y^{2}\right) y_{i}$ and $\alpha_{w}: V \rightarrow V$ has 
non-zero image in $\pi_{i} V$ for each $w \in S$. Choose an element $w \in S$ whose expression in reduced form as a product of letters $y_{i}^{ \pm}$is of maximal length. We consider two cases:

Case 1: $w=w^{\prime} y_{i}$ for some $w^{\prime} \in F_{\mu}\left(y^{2}\right)$ and some $i$. The equation

$$
\left((1-\rho(s)) y^{-1}+\rho(s) y\right) \alpha=1
$$

implies that $\rho\left(s \pi_{j}\right) \alpha_{w}=0$ for each $j$. Any element $x$ in the image of $\alpha_{w}$ satisfies the conditions of Lemma 5.23 so $(V, \rho)$ has a non-zero submodule $\left(V^{\prime}, \rho^{\prime}\right)$ with $\rho^{\prime}(s)=0$.

Case 2: $w=w^{\prime} y_{i}^{-1}$ for some $w^{\prime} \in F_{\mu}\left(y^{2}\right)$. The equation (42) implies that $\rho\left((1-s) \pi_{i}\right) \alpha_{w}=0$ for each $i$. By Lemma 5.23 there is a non-zero submodule $\left(V^{\prime}, \rho^{\prime}\right)$ with $\rho^{\prime}(1-s)=0$ or in other words $\rho^{\prime}(s)=1$.

Recall that a module $V$ is called simple if there are no submodules other than 0 and $V$. The following remark is a consequence of Lemma 5.24.

Remark 5.25. Every simple primitive module is trivially primitive.

Definition 5.26. If $\mathcal{A}$ is an abelian category then a non-empty full subcategory $\mathcal{E} \subset \mathcal{A}$ is called a Serre subcategory if for every exact sequence $0 \rightarrow V \rightarrow V^{\prime} \rightarrow V^{\prime \prime} \rightarrow 0$ in $\mathcal{A}$ one has

$$
V^{\prime} \in \mathcal{E} \Leftrightarrow V \in \mathcal{E} \text { and } V^{\prime \prime} \in \mathcal{E} .
$$

Note that every Serre subcategory of an abelian category is again an abelian category. Since $B$ preserves exact sequences and arbitrary direct sums $\mathcal{P r i m}_{\infty}(A)$ is a Serre subcategory of $\mathcal{S e i}_{\infty}(A)$ and is closed under direct sums.

Lemma 5.27. Suppose $\mathcal{E} \subset \mathcal{A}$ is a Serre subcategory of an abelian category and $\mathcal{E}$ is closed under arbitrary direct sums. Every module $V \in \mathcal{A}$ contains a unique maximal submodule $U \leq V$ such that $U \in \mathcal{E}$. If $U^{\prime} \leq V$ and $U^{\prime} \in \mathcal{E}$ then $U^{\prime} \leq U$.

Proof. Let $U$ be the sum in $V$ of all the submodules $U_{i} \leq V$ with $U_{i} \in \mathcal{E}$. Since $U$ is a factor module of $\bigoplus U_{i}$, one finds $U \in \mathcal{E}$.

Proposition 5.28. The category $\mathcal{P r i m}_{\infty}(A)$ is the smallest Serre subcategory of $\mathcal{S e i}_{\infty}(A)$ which a) contains the trivially primitive modules and $\left.b\right)$ is closed under arbitrary direct sums. 
Proof. Let $\mathcal{P}_{\infty}(A)$ denote the smallest full subcategory of $\mathcal{S} \mathrm{ei}_{\infty}(A)$ satisfying the conditions of the Proposition. Now $\mathcal{P r i m}_{\infty}(A)$ satisfies these conditions so $\mathcal{P}_{\infty}(A) \subset \mathcal{P} \operatorname{rim}_{\infty}(A)$.

Conversely, we must show that $\operatorname{Prim}_{\infty}(A) \subset \mathcal{P}_{\infty}(A)$. Suppose $B(V)=$ 0 . Let $W \leq V$ be the maximal submodule such that $W \in \mathcal{P}_{\infty}(A)$ (the module $W$ exists by Lemma 5.27). Now $B(V / W)=0$ since $B$ respects exact sequences so Lemma 5.24 implies that either $V / W=0$ or there is a non-zero submodule $V^{\prime}$ of $V / W$ which lies in $\mathcal{P}_{\infty}(A)$. In the latter case, let $p: V \rightarrow V / W$ denote the projection and note the exact sequence

$$
0 \rightarrow W \rightarrow p^{-1}\left(V^{\prime}\right) \stackrel{p \mid}{\rightarrow} V^{\prime} \rightarrow 0
$$

Since $W \in \mathcal{P}_{\infty}(A)$ and $V^{\prime} \in \mathcal{P}_{\infty}(A)$ we have $p^{-1}(V) \in \mathcal{P}_{\infty}(A)$ which contradicts the maximality of $W$. Thus $V / W=0$ and hence $V=W$, so $V$ lies in $\mathcal{P}_{\infty}(A)$.

\subsection{Construction of the quotient}

We outline next a construction of $\mathcal{S} \operatorname{ei}_{\infty}(A) / \mathcal{P} \operatorname{rim}_{\infty}(A)$. We will use this construction in Section 6 to show that $B: W^{\zeta}(\mathcal{S} \operatorname{ei}(A)) \rightarrow W^{-\zeta}(\mathcal{F} \operatorname{lk}(A))$ is an isomorphism when $A$ is a semi-simple Artinian ring. The notion of Serre subcategory was defined in the preceding section. Let us note some basic properties:

Lemma 5.29. Suppose $\mathcal{A}$ is an abelian category and $\mathcal{E}$ is a Serre subcategory. Suppose $V \in \mathcal{A}, W \leq V$ and $W^{\prime} \leq V$.

1. If $W \in \mathcal{E}$ and $W^{\prime} \in \mathcal{E}$ then $W+W^{\prime} \in \mathcal{E}$.

2. If $V / W \in \mathcal{E}$ and $V / W^{\prime} \in \mathcal{E}$ then $V /\left(W \cap W^{\prime}\right) \in \mathcal{E}$.

Proof. 1. There is an exact sequence

$$
0 \rightarrow W \rightarrow W+W^{\prime} \rightarrow\left(W+W^{\prime}\right) / W \rightarrow 0
$$

and $\left(W+W^{\prime}\right) / W$ is isomorphic to $W^{\prime} /\left(W \cap W^{\prime}\right) \in \mathcal{E}$. Hence $W+W^{\prime} \in \mathcal{E}$. 2. There is an exact sequence

$$
0 \rightarrow W /\left(W \cap W^{\prime}\right) \rightarrow V /\left(W \cap W^{\prime}\right) \rightarrow V / W \rightarrow 0 .
$$

Now $W /\left(W \cap W^{\prime}\right)$ is isomorphic to $\left(W+W^{\prime}\right) / W^{\prime}$ which is contained in $V / W^{\prime}$ and so $W /\left(W \cap W^{\prime}\right) \in \mathcal{E}$ and hence $V / W \cap W^{\prime} \in \mathcal{E}$. 
We may now recall a construction for the quotient of an abelian category by a Serre subcategory. See Gabriel [20] or Srinivas [53, Appendix B.3] for further details.

Suppose $\mathcal{A}$ is an abelian category and $\mathcal{E}$ is a Serre subcategory. The symbol $\mathcal{A} / \mathcal{E}$ will denote a category with the same objects as $\mathcal{A}$ but different groups of morphisms. To define $\operatorname{Hom}_{\mathcal{A} / \mathcal{E}}\left(V, V^{\prime}\right)$, consider the pairs $\left(W, U^{\prime}\right)$ where $W \leq V, U^{\prime} \leq V^{\prime}, V / W \in \mathcal{E}$ and $U^{\prime} \in \mathcal{E}$. One says that $\left(W_{1}, U_{1}^{\prime}\right) \leq\left(W_{2}, U_{2}^{\prime}\right)$ if $W_{2} \leq W_{1}$ and $U_{1}^{\prime} \leq U_{2}^{\prime}$ [note the directions of inclusion]. Lemma 5.29 above implies that these pairs are a directed set. Indeed, given pairs $\left(W_{1}, U_{1}^{\prime}\right)$ and $\left(W_{2}, U_{2}^{\prime}\right)$ one finds $\left(W_{1}, U_{1}^{\prime}\right) \leq\left(W_{1} \cap W_{2}, U_{1}^{\prime}+U_{2}^{\prime}\right)$ and $\left(W_{2}, U_{2}^{\prime}\right) \leq\left(W_{1} \cap W_{2}, U_{1}^{\prime}+U_{2}^{\prime}\right)$. The following definition can now be made:

$$
\operatorname{Hom}_{\mathcal{A} / \mathcal{E}}\left(V, V^{\prime}\right)=\underset{\left(\overrightarrow{W, U^{\prime}}\right)}{\lim _{\mathcal{A}}} \operatorname{Hom}_{\mathcal{A}}\left(W, V^{\prime} / U^{\prime}\right)
$$

We leave to the reader the definition of composition of morphisms and the canonical functor $F: \mathcal{A} \rightarrow \mathcal{A} / \mathcal{E}$. Proofs of the following statements can be found in the references cited above:

(a) The quotient category $\mathcal{A} / \mathcal{E}$ is an abelian category and $F$ is an exact additive functor.

(b) If $f$ is a morphism in $\mathcal{A}$ then $F(f)$ is an isomorphism if and only if $\operatorname{Coker}(f) \in \mathcal{E}$ and $\operatorname{Ker}(f) \in \mathcal{E}$.

In particular if $V$ is an object in $\mathcal{A}$ then $F(V) \cong 0$ if and only if $V \in \mathcal{E}$.

As we indicated in earlier sections, the functor $F: \mathcal{A} \rightarrow \mathcal{A} / \mathcal{E}$ is universal with respect to property (b). In detail, if $G: \mathcal{A} \rightarrow \mathcal{B}$ makes invertible every morphism whose kernel and cokernel lie in $\mathcal{E}$ then there is a unique functor $\widetilde{G}: \mathcal{A} / \mathcal{E} \rightarrow \mathcal{B}$ such that $\widetilde{G} F=G$. In particular the functor

$$
F: \mathcal{S e i}_{\infty}(A) \rightarrow \mathcal{S} \operatorname{ei}_{\infty}(A) / \mathcal{P} \operatorname{rim}_{\infty}(A)
$$

satisfies Definition 5.13. Let us be explicit about $\widetilde{G}$ :

If $V$ is an object in $\mathcal{A} / \mathcal{E}$ then one writes $\widetilde{G}(V)=G(V)$. Every morphism $f \in \operatorname{Hom}_{\mathcal{A} / \mathcal{E}}\left(V, V^{\prime}\right)$ is represented by some $\bar{f} \in \operatorname{Hom}_{\mathcal{A}}\left(W, V^{\prime} / U^{\prime}\right)$ with $U^{\prime} \in$ $\mathcal{E}$ and $V / W \in \mathcal{E}$ If $i: W \rightarrow V$ and $p: V^{\prime} \rightarrow V^{\prime} / U^{\prime}$ denote the canonical monomorphism and epimorphism respectively one must define

$$
\widetilde{G}(f)=G(p)^{-1} G(\bar{f}) G(i)^{-1}: G(V) \rightarrow G\left(V^{\prime}\right) .
$$

In our particular example Lemma 5.27 provides one simplification in our description of the quotient category $\mathcal{S e i}_{\infty}(A) / \mathcal{P} \operatorname{rim}_{\infty}(A)$. If $V \in \mathcal{S} \operatorname{ei}_{\infty}(A)$ let us call a submodule $W \leq V$ coprimitive if $V / W \in \mathcal{P}_{\infty}(A)$. 
Lemma 5.30. If $V, V^{\prime} \in \mathcal{S} \operatorname{ei}_{\infty}(A)$ and $U^{\prime}$ denotes the maximal primitive submodule of $V^{\prime}$ then

$$
\operatorname{Hom}_{\mathcal{S} \mathrm{ei}_{\infty}(A) / \mathcal{P} \operatorname{rim}_{\infty}(A)}\left(V, V^{\prime}\right)=\underset{W}{\lim _{W}} \operatorname{Hom}_{\mathcal{S e i}_{\infty}(A)}\left(W, V^{\prime} / U^{\prime}\right)
$$

where the direct limit is over coprimitive submodules $W$ of $V$.

Note that there is not in general a minimal coprimitive in $V$; the functor $B$ does not respect infinite limits and an infinite intersection of coprimitives is not in general coprimitive (but see Lemma 5.31 below).

\subsection{Global dimension zero}

In this section the ring $A$ will be assumed semi-simple and Artinian or, in other words, a finite product of matrix rings over division rings. The basic theory of semi-simple Artinian rings can be found in many algebra textbooks (e.g. Lam [29, §1-4] or Lang [30, Ch.XVII]). In particular, all $A$-modules are projective and $\mathcal{S}$ ei $(A)$ is an abelian category with ascending and descending chain conditions; these facts lead to simplifications of results in Sections 5.5 and 5.6 above. We show that the primitive modules in $\mathcal{S}$ ei $(A)$ are composed of a finite number of simple trivially primitive modules (Proposition 5.33) and give a simplified description of the hermitian category $\mathcal{S}$ ei $(A) / \mathcal{P} \operatorname{rim}_{\infty}(A)$. We shall consider semi-simple Artinian rings again in Section 6 but it is not essential to read the present section before Section 6 .

The key lemma we will need is the following:

Lemma 5.31. Suppose $\mathcal{A}$ is an abelian category with ascending and descending chain conditions and $\mathcal{E}$ is a Serre subcategory.

1. Every module $V \in \mathcal{A}$ contains a unique maximal submodule in $\mathcal{E}$ which contains all others in $\mathcal{E}$.

2. Every module $V \in \mathcal{A}$ contains a unique submodule $W \leq V$ which is minimal with respect to the property $V / W \in \mathcal{E}$. If $V / W^{\prime} \in \mathcal{E}$ then $W \leq W^{\prime}$.

Proof. 1. Since $\mathcal{A}$ has the ascending chain condition there is a submodule $U \leq V$ which is maximal with respect to the property $U \in \mathcal{E}$. In other words, if $U \leq U^{\prime} \leq V$ and $U^{\prime} \in \mathcal{E}$ then $U^{\prime}=U$. If $U^{\prime}$ is any other submodule in $\mathcal{E}$ then $U+U^{\prime} \in \mathcal{E}$ by Lemma 5.29 so $U+U^{\prime}=U$ and hence $U^{\prime} \leq U$.

2. Since $\mathcal{A}$ has the descending chain condition there is a submodule $W \leq V$ which is minimal with respect to the property $V / W \in \mathcal{E}$ (i.e. if $W^{\prime} \leq W \leq V$ 
and $V / W^{\prime} \in \mathcal{E}$ then $\left.W^{\prime}=W\right)$. If $V / W^{\prime} \in \mathcal{E}$ then $V /\left(W \cap W^{\prime}\right) \in \mathcal{E}$ by Lemma 5.29 so $W \cap W^{\prime}=W$ and hence $W \leq W^{\prime}$.

Recall that if $V \in \mathcal{S}$ ei $(A)$, a submodule $W \leq V$ is called coprimitive if $V / W$ is primitive. Since $A$ is Artinian and Noetherian, Lemma 5.31 implies that there is a maximal primitive submodule $U \leq V$ and a minimal coprimitive submodule $W \leq V$ for each $V \in \mathcal{S}$ ei $(A)$.

\subsubsection{Structure of Primitives}

Definition 5.32. Let $\mathcal{P} \operatorname{rim}(A)$ denote the intersection of $\mathcal{P r i m}_{\infty}(A)$ and $\mathcal{S}$ ei $(A)$. In other words, $\operatorname{Prim}(A) \subset \mathcal{S} \operatorname{ei}(A)$ is the full subcategory containing those modules $V$ such that $B(V)=0$.

Note that $\mathcal{P} \operatorname{rim}(A)$ is both a Serre subcategory and a hermitian subcategory of $\mathcal{S}$ ei $(A)$. Moreover, $\mathcal{S} \operatorname{ei}(A) / \mathcal{P} \operatorname{rim}(A)=\mathcal{S} \operatorname{ei}(A) / \mathcal{P} \operatorname{rim}_{\infty}(A)$.

Proposition 5.33. The category $\mathcal{P} \operatorname{rim}(A)$ is the smallest Serre subcategory of $\mathcal{S} \mathrm{ei}(A)$ which contains the trivially primitive modules in $\mathcal{S} \operatorname{ei}(A)$.

Proof. We proceed as in the proof of Proposition 5.28, using Lemma 5.31 in place of Lemma 5.27. Let $\mathcal{P}(A)$ denote the smallest Serre subcategory of $\mathcal{S}$ ei $(A)$ which contains all the trivially primitive modules. To show that $\mathcal{P}(A) \subset \mathcal{P} \operatorname{rim}(A)$ it suffices to observe that $\operatorname{Prim}(A)$ is a Serre subcategory which contains these modules.

Conversely, to show $\mathcal{P} \operatorname{rim}(A) \subset \mathcal{P}(A)$ suppose $V \in \mathcal{P} \operatorname{rim}(A)$. There exists, by Lemma 5.31, a maximal submodule $U \leq V$ such that $U \in \mathcal{P}(A)$. Now $B(V / U)=0$ so either $V=U$ or by Lemma $5.24 V / U$ has a nonzero trivially primitive submodule $U^{\prime}$. If $p: V \rightarrow V / U$ is the canonical map then the exact sequence $0 \rightarrow U \rightarrow p^{-1}\left(U^{\prime}\right) \rightarrow U^{\prime} \rightarrow 0$ implies that $p^{-1}\left(U^{\prime}\right) \in \mathcal{P}(A)$ contradicting the maximality of $U$. Thus $V=U$ and $V \in \mathcal{P}(A)$.

\subsubsection{Construction of the quotient}

With the benefit of Lemma 5.31 we can give a simpler description of the quotient category than Lemma 5.30.

Lemma 5.34. The morphisms in $\mathcal{S e i}(A) / \mathcal{P} \operatorname{rim}(A)$ are

$$
\operatorname{Hom}_{\mathcal{S e i}(A) / \mathcal{P} \operatorname{rim}(A)}\left(V, V^{\prime}\right)=\operatorname{Hom}_{\mathcal{S e i}(A)}\left(W, V^{\prime} / U^{\prime}\right) .
$$

where $W \leq V$ is the minimal coprimitive and $U^{\prime} \leq V^{\prime}$ is the maximal primitive. 
We simplify next the hermitian structure on $\mathcal{S} \operatorname{ei}(A) / \mathcal{P} \operatorname{rim}(A)$. We have seen that the duality-preserving functor $(B, \Phi,-1): \mathcal{S} \operatorname{ei}(A) \rightarrow \mathcal{F} \operatorname{lk}(A)$ factors through an equivalence of hermitian categories $(\bar{B}, \Phi,-1)$ :

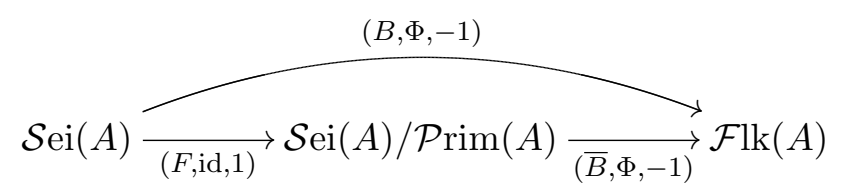

(Theorem 5.17 and Proposition 5.21 above). The duality functor on the quotient $\mathcal{S} \operatorname{ei}(A) / \mathcal{P} \operatorname{rim}(A)$ was defined in Section 5.4 above by $F(V)^{*}=$ $F\left(V^{*}\right)$ and by equation (40). Using the assumption that $A$ is Artinian we can re-interpret equation (40). Suppose that $f \in \operatorname{Hom}_{\mathcal{S e i}(A) / \mathcal{P} \operatorname{rim}(A)}\left(V, V^{\prime}\right)$. As usual, let $W$ denote the minimal coprimitive submodule of $V$ and let $U^{\prime}$ denote the maximal primitive submodule of $V^{\prime}$. The morphism $f$ is identified with some $\bar{f} \in \operatorname{Hom}_{\mathcal{S e i}(A)}\left(W, V^{\prime} / U^{\prime}\right)$. Since ${ }^{*}$ preserves exact sequences the following are exact

$$
\begin{aligned}
& 0 \rightarrow(V / W)^{*} \rightarrow V^{*} \rightarrow W^{*} \rightarrow 0 \\
& 0 \rightarrow\left(V^{\prime} / U^{\prime}\right)^{*} \rightarrow\left(V^{\prime}\right)^{*} \rightarrow\left(U^{\prime}\right)^{*} \rightarrow 0 .
\end{aligned}
$$

Now $(B, \Phi, 1)$ is a duality-preserving functor, so for each $V \in \mathcal{S} \operatorname{si}(A)$ one has $B(V)=0$ if and only if $B\left(V^{*}\right)=0$. Thus $\operatorname{Prim}(A)$ is a hermitian subcategory of $\mathcal{S} \mathrm{ei}(A)$ and in particular $\left(U^{\prime}\right)^{*}$ and $(V / W)^{*}$ are primitive. It follows that $(V / W)^{*}$ is the maximal primitive in $V^{*}$ and $\left(V^{\prime} / U^{\prime}\right)^{*}$ is the minimal coprimitive in $\left(V^{\prime}\right)^{*}$. Since $(F$, id, 1$)$ is a duality-preserving functor, $f^{*}$ is represented by

$$
\bar{f}^{*} \in \operatorname{Hom}\left(\left(V^{\prime} / U^{\prime}\right)^{*}, W^{*}\right) .
$$

\section{Equivalence of Invariants}

Cobordism invariants of $F_{\mu}$-links have been defined in two different ways in Sections 3 and [51]. In this section we use the duality-preserving functor $(B, \Phi,-1)$ which was studied in Section 5 to relate the two approaches, proving Theorems 1.3 and 1.4. To prove Theorem 1.4 we show that the functor $B$ respects each of the three steps laid out in Sections 3.1 and 4.3. A more detailed version of Theorem 1.4 is set out in Theorem 6.5 below.

\subsection{Proof of Theorem 1.3}

Suppose $\mathcal{A}$ is an abelian category with ascending and descending chain conditions and $\mathcal{E}$ is a Serre subcategory. Let $F: \mathcal{A} \rightarrow \mathcal{A} / \mathcal{E}$ denote the quotient 
functor. Recall that a module $V$ in $\mathcal{A}$ is called simple if $V$ is not isomorphic to 0 and $V$ does not have submodules other than 0 and $V$.

Lemma 6.1. 1. If $V \in \mathcal{A}$ is simple then either $V \in \mathcal{E}$ or $F(V)$ is simple.

2. Every simple module in $\mathcal{A} / \mathcal{E}$ is isomorphic to $F(V)$ for some simple module $V \in \mathcal{A}$ which does not lie in $\mathcal{E}$.

Proof. 1. Suppose $V \in \mathcal{A}$ is simple, $V \notin \mathcal{E}$ and $i: V^{\prime} \rightarrow F(V)$ is the inclusion of a submodule in $\mathcal{A} / \mathcal{E}$. Now $i$ is represented by some morphism $\bar{i}: W^{\prime} \rightarrow V$ where $W^{\prime} \leq V^{\prime}$ and $V^{\prime} / W^{\prime} \in \mathcal{E}$. Either $\bar{i}=0$ in which case $V^{\prime} \cong 0$ in $\mathcal{A} / \mathcal{E}$ or $\bar{i}$ is an epimorphism which implies that $V^{\prime}=F(V)$ (recall that $F$ is exact). 2. Every module in $\mathcal{A} / \mathcal{E}$ is $F(V)$ for some module $V \in \mathcal{A}$. Suppose $F(V)$ is simple. Now $V$ has a finite filtration $0=V_{0} \leq V_{1} \leq \cdots \leq V_{n}=V$ where each quotient $V_{i} / V_{i-1}$ is a simple module. Since $F$ respects exact sequences $F\left(V_{i} / V_{i-1}\right)=0$ for all $i \in\{1, \cdots, n\}$ except one, for which there is an isomorphism $F\left(V_{i} / V_{i-1}\right) \cong F(V)$. This module $V_{i} / V_{i-1}$ does not lie in $\mathcal{E}$.

Suppose now that $\mathcal{A}$ and $\mathcal{A} / \mathcal{E}$ are hermitian categories and the quotient functor extends to a duality-preserving functor

$$
(F, \mathrm{id}, 1): \mathcal{A} \rightarrow \mathcal{A} / \mathcal{E} .
$$

Lemma 6.2. $\quad$ 1. The Serre subcategory $\mathcal{E}$ is a hermitian subcategory.

2. Let $\zeta=1$ or -1 . If $V \in \mathcal{A}$ is simple and $V \notin \mathcal{E}$ then $V$ is $\zeta$-self-dual if and only if $F(V)$ is $\zeta$-self-dual.

Proof. 1. If $V \in \mathcal{E}$ then $F\left(V^{*}\right)=F(V)^{*} \cong 0 \in \mathcal{A} / \mathcal{E}$ so $V^{*} \in \mathcal{E}$.

2. To prove the "only if" part it suffices to recall that for $\phi: V \rightarrow V^{*}$ one has $F\left(\phi^{*}\right)=F(\phi)^{*}$. For the "if" part, note also that

$$
F: \operatorname{Hom}_{\mathcal{A}}\left(V, V^{*}\right) \rightarrow \operatorname{Hom}_{\mathcal{A} / \mathcal{E}}\left(V, V^{*}\right)
$$

is an isomorphism.

Proposition 6.3. Suppose $\mathcal{A}$ and $\mathcal{A} / \mathcal{E}$ are hermitian categories and

$$
(F, \text { id, } 1): \mathcal{A} \rightarrow \mathcal{A} / \mathcal{E}
$$

is a duality-preserving functor. For each $\zeta$-self-dual simple module $V \in \mathcal{A}$ such that $V \notin \mathcal{E}$ there is a canonical isomorphism

$$
W^{\zeta}\left(\left.\mathcal{A}\right|_{V}\right) \cong W^{\zeta}\left(\left.(\mathcal{A} / \mathcal{E})\right|_{V}\right)
$$


If $\mathcal{A}$ has ascending and descending chain conditions then there is a canonical isomorphism

$$
W^{\zeta}(\mathcal{A}) \cong W^{\zeta}(\mathcal{E}) \oplus W^{\zeta}(\mathcal{A} / \mathcal{E})
$$

Proof. If $V \in \mathcal{A}$ is a simple module and $V \notin \mathcal{E}$ then $F(V)$ is simple by part 1. of Lemma 6.1. Every module in $\left.\mathcal{A}\right|_{V}$ is a direct sum of copies of $V$ so by equation (43) the restriction $F:\left.\left.\mathcal{A}\right|_{V} \rightarrow(\mathcal{A} / \mathcal{E})\right|_{F(V)}$ is a full and faithful functor and hence an equivalence of categories.

By part 2. of Lemma 6.2, $V$ is $\zeta$-self-dual if and only if $F(V)$ is $\zeta$-selfdual, in which case $(F$, id, 1$): F:\left.\left.\mathcal{A}\right|_{V} \rightarrow(\mathcal{A} / \mathcal{E})\right|_{F(V)}$ is an equivalence of hermitian categories and induces an isomorphism

$$
W^{\zeta}\left(\left.\mathcal{A}\right|_{V}\right) \rightarrow W^{\zeta}\left(\left.(\mathcal{A} / \mathcal{E})\right|_{F(V)}\right)
$$

To prove the last sentence of the Lemma, note first that by part 1 . of Lemma $6.2, \mathcal{E}$ is a hermitian subcategory of $\mathcal{A}$. Theorem 3.4 provides canonical decompositions

$$
\begin{aligned}
& W^{\zeta}(\mathcal{A}) \cong \bigoplus W^{\zeta}\left(\left.\mathcal{A}\right|_{V}\right) \\
& W^{\zeta}(\mathcal{E}) \cong \bigoplus W^{\zeta}\left(\left.\mathcal{E}\right|_{V}\right) \\
& W^{\zeta}(\mathcal{A} / \mathcal{E}) \cong \bigoplus W^{\zeta}\left(\left.(\mathcal{A} / \mathcal{E})\right|_{V}\right)
\end{aligned}
$$

where the right hand side of each identity has one summand for each isomorphism class of $\zeta$-self-dual simple modules $V$.

By part 2. of Lemma 6.1 and part 2. of Lemma 6.2 every summand of $W^{\zeta}(\mathcal{A} / \mathcal{E})$ is the isomorphic image of $W^{\zeta}\left(\left.\mathcal{A}\right|_{V}\right)$ for some simple $\zeta$-self-dual module $V$ in $\mathcal{A}$.

On the other hand, if $V \in \mathcal{E}$ is simple and $\zeta$-self-dual then $(F$, id, 1$)$ sends $W^{\zeta}\left(\left.\mathcal{A}\right|_{V}\right)$ to zero. The last sentence of the Lemma follows.

In our application, we set $\mathcal{A}=\mathcal{S} \operatorname{ei}(A)$ and $\mathcal{E}=\mathcal{P} \operatorname{rim}(A)$ where $A$ is semi-simple Artinian. Recall that $\mathcal{P} \operatorname{rim}(A)=\mathcal{P}_{\operatorname{rim}}(A) \cap \mathcal{S} \operatorname{ei}(A)$ is an abelian category with ascending and descending chain conditions and $\mathcal{S}$ ei $(A) / \mathcal{P} \operatorname{rim}(A)=\mathcal{S} \operatorname{ei}(A) / \mathcal{P} \operatorname{rim}_{\infty}(A)$.

Lemma 6.4. 1. None of the simple primitive modules in $\operatorname{Prim}(A)$ are self-dual.

2. $W^{\zeta}(\mathcal{P} \operatorname{rim}(A))=0$. 
Proof. By Remark 5.25 above, every simple primitive module is trivially primitive. If $(V, \rho) \in \mathcal{P} \operatorname{rim}(A)$ then $\rho(s)=0$ if and only if $\rho^{*}(s)=1$ so none of the simple trivially primitive modules are self-dual. Thus part 1 . is proved, and part 2. follows immediately from Theorem 3.4.

Proof of Theorem 1.3. By Proposition 5.21 the duality-preserving functor $(B, \Phi,-1)$ is the composite $(\bar{B}, \Phi,-1) \circ(F, \mathrm{id}, 1)$. Setting $\mathcal{A}=\mathcal{S} \operatorname{ei}(A)$ and $\mathcal{E}=\operatorname{Prim}(A)$ in Proposition 6.3, and invoking also Lemma 6.4, we learn that $(F$, id, 1$)$ induces an isomorphism

$$
W^{\zeta}(\mathcal{S} \operatorname{ei}(A)) \rightarrow W^{\zeta}(\mathcal{S} \operatorname{ei}(A) / \mathcal{P} \operatorname{rim}(A)) .
$$

By Theorem 5.17, $(\bar{B}, \Phi, 1)$ is an equivalence and hence induces an isomorphism

$$
W^{\zeta}(\mathcal{S} \operatorname{ei}(A) / \mathcal{P} \operatorname{rim}(A)) \rightarrow W^{-\zeta}(\mathcal{F} \operatorname{lk}(A)) .
$$

(see Lemma A.2 in Appendix A). Thus $(B, \Phi,-1)$ induces an isomorphism

$$
W^{\zeta}(\mathcal{S} \operatorname{ei}(A)) \rightarrow W^{-\zeta}(\mathcal{F} \operatorname{lk}(A)) .
$$

This completes the proof of Theorem 1.3.

\subsection{Proof of Theorem 1.4}

We prove in this section that the functor $(B, \Phi,-1)$ identifies the invariants defined in [51] with those of Section 3. More precisely, we prove the following theorem:

\section{Theorem 6.5. (Equivalence of invariants)}

1. If $V \in \mathcal{S}$ ei $(\mathbb{Q})$ is simple and $(-1)^{q}$-self-dual then $B(V) \in \mathcal{F} \operatorname{lk}(\mathbb{Q})$ is simple and $(-1)^{q+1}$-self-dual.

2. Every simple $(-1)^{q+1}$-self-dual module $M \in \mathcal{F} \operatorname{lk}(\mathbb{Q})$ is isomorphic to $B(V)$ for some simple $(-1)^{q}$-self-dual module $V \in \mathcal{S}$ ei $(\mathbb{Q})$.

3. If $V \in \mathcal{S} \mathrm{ei}(\mathbb{Q})$ and $B(V) \in \mathcal{F} \operatorname{lk}(\mathbb{Q})$ are simple then the functor $B$ induces an isomorphism of rings $B: \operatorname{End}_{\mathcal{S e i}(\mathbb{Q})}(V) \stackrel{\cong}{\rightrightarrows} \operatorname{End}_{\mathcal{F l k}(\mathbb{Q})}(B(V))$.

4. Suppose $V \in \mathcal{S} \operatorname{ei}(\mathbb{Q})$ is simple and $b: V \rightarrow V^{*}$ is a $\zeta$-hermitian form. The ring isomorphism in part 3. respects involutions. Explicitly, if $f \in \operatorname{End}_{\mathcal{S e i}(\mathbb{Q})} V$ then $B\left(b^{-1} f^{*} b\right)=\left(\Phi_{V} B(b)\right)^{-1} B(f)^{\wedge} \Phi_{V} B(b)$. 
5. Suppose $W \in \mathcal{S}$ ei $(\mathbb{Z})$ and $\phi: W \rightarrow W^{*}$ is a $(-1)^{q}$-hermitian form. The dimension modulo 2, signatures, discriminant, Hasse-Witt invariant and Lewis $\theta$-invariant of

$$
\Theta_{V, b} p_{V}\left[\mathbb{Q} \otimes_{\mathbb{Z}}(W, \phi)\right] \in W^{1}\left(\operatorname{End}_{\mathcal{S e i}(\mathbb{Q})}(V)\right)
$$

coincide (if defined) with the corresponding invariants of

$$
\Theta_{\left(B(V),-\Phi_{V} B(b)\right)} p_{B(V)}\left[\mathbb{Q} \otimes_{\mathbb{Z}}\left(B(W), \Phi_{W} B(\phi)\right)\right] \in W^{1}\left(\operatorname{End}_{\mathcal{F} l \mathrm{k}(\mathbb{Q})}(B(V))\right) .
$$

Recall that $B=\bar{B} \circ F$ and $\bar{B}: \mathcal{S} \operatorname{ei}(\mathbb{Q}) / \mathcal{P} \operatorname{rim}(\mathbb{Q}) \rightarrow \mathcal{F} \operatorname{lk}(\mathbb{Q})$ is an equivalence of categories. In parts 1 . through 3. of Theorem 6.5 it therefore suffices to prove corresponding statements with the functor $F$ in place of $B$ and $(-1)^{q}$ in place of $(-1)^{q+1}$ :

1. The statement follows from part 1. of Lemma 6.1, part 2. of Lemma 6.2 and part 1. of Lemma 6.4.

2. The statement follows from part 2. of Lemma 6.1 and part 2. of Lemma 6.2.

3. This is a consequence of equation (44).

4. Since $B\left(b^{-1} f^{*} b\right)=B\left(b^{-1}\right) B\left(f^{*}\right) B(b)$ it suffices to prove that

$$
B\left(f^{*}\right)=\Phi_{V}^{-1} B(f)^{\wedge} \Phi_{V} .
$$

This equation is a consequence of the fact that $\Phi$ is a natural isomorphism.

The proof of part 5 . of Theorem 6.5 is slightly more involved. Recall from proposition 5.7 that $B$ respects changes of coefficients and, in particular, that the inclusion of $\mathbb{Z}$ in $\mathbb{Q}$ induces the commutative diagram (24). One must check that $(B, \Phi,-1)$ respects each of the three steps in the definitions of the $F_{\mu}$-link invariants (see Section 3.1).

Devissage: Let $V \in \mathcal{S}$ ei $(\mathbb{Q})$ be a $\zeta$-self-dual simple module. If $W$ is isomorphic to a direct sum of copies of $V$ then $B(W)$ is isomorphic to a direct sum of copies of $B(V)$. Hence the image of $W^{\zeta}\left(\mathcal{S}\right.$ ei $\left.\left.(\mathbb{Q})\right|_{V}\right)$ under $B$ lies in $W^{-\zeta}\left(\left.\mathcal{F} \operatorname{lk}(\mathbb{Q})\right|_{B(V)}\right)$ and there is a commutative diagram of isomorphisms

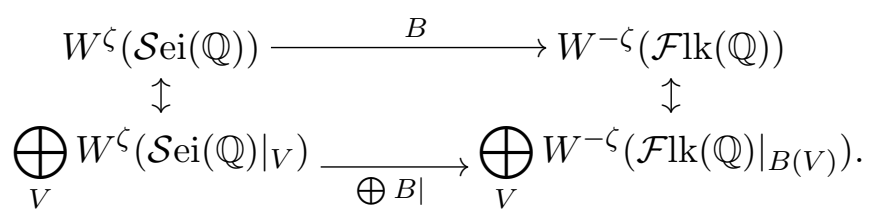

where the direct sums are indexed by the isomorphism classes of simple $\zeta$-self-dual modules in $\mathcal{S}$ ei $(\mathbb{Q})$.

Morita Equivalence: Suppose $V \in \mathcal{S}$ ei $(\mathbb{Q})$ is a simple module and

$$
b: V \rightarrow V^{*}
$$


is a non-singular $\zeta$-hermitian form. Let us denote the endomorphism rings $E=\operatorname{End}_{\mathcal{S e i}(\mathbb{Q})} V$ and $E^{\prime}=\operatorname{End}_{\mathcal{F} l \mathrm{k}(\mathbb{Q})} B(V)$. By Corollary 3.23 above the duality-preserving functor $(B, \Phi,-1)$ induces a commutative diagram

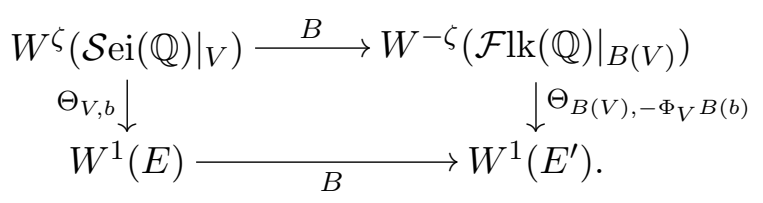

Invariants: The isomorphism $E \rightarrow E^{\prime}$ in part 4. induces isomorphisms between the target groups for the invariants in part 5. For example, if $E$ and $E^{\prime}$ are commutative with trivial involution then the discriminant $\Delta$ of $\Theta_{V, b} p_{V}\left[\mathbb{Q} \otimes_{\mathbb{Z}}(W, \phi)\right]$ lies in $E / E^{2}$ and the functor $B$ induces an isomorphism $E / E^{2} \rightarrow E^{\prime} /\left(E^{\prime}\right)^{2}$. The word "coincide" in part 5 . is understood to mean that the image of $\Delta$ in $E^{\prime} /\left(E^{\prime}\right)^{2}$ is equal to the discriminant of $\Theta_{\left(B(V),-\Phi_{V} B(b)\right)} p_{B(V)}\left[\mathbb{Q} \otimes_{\mathbb{Z}}\left(B(W), \Phi_{W} B(\phi)\right)\right]$.

The isomorphism $B: E \rightarrow E^{\prime}$ of rings with involution induces an isomorphism $W^{1}(E) \rightarrow W^{1}\left(E^{\prime}\right)$. We leave it to the reader to check that if $\alpha \in W^{1}(E)$ then all the listed invariants of $\alpha$ coincide (in this sense) with the corresponding invariants of $B(\alpha) \in W^{1}\left(E^{\prime}\right)$. Further details of the invariants can be found in chapter 11 of [51].

This completes the proof of part 5. and hence of theorems 1.4 and 6.5.

\section{A Naturality of constructions}

In this appendix we prove naturality theorems for the covering construction $(B, \Phi,-1)$ and for hermitian Morita equivalence, proving Propositions 5.7 and 3.22 above.

To compare duality-preserving functors one requires the following definition.

Definition A.1. Suppose $(G, \Psi, \eta): \mathcal{C} \rightarrow \mathcal{D}$ and $\left(G^{\prime}, \Psi^{\prime}, \eta\right): \mathcal{C} \rightarrow \mathcal{D}$ are duality-preserving functors between hermitian categories $\mathcal{C}$ and $\mathcal{D}$. A natural transformation $\alpha:(G, \Psi, \eta) \rightarrow\left(G^{\prime}, \Psi^{\prime}, \eta\right)$ is a natural transformation between the underlying functors, $\alpha: G \rightarrow G^{\prime}$, such that

$$
\Psi_{V}=\alpha_{V}^{*} \Psi_{V}^{\prime} \alpha_{V^{*}}
$$

for each object $V \in \mathcal{C}$. 
If $\alpha: G \rightarrow G^{\prime}$ is a natural isomorphism between the underlying functors and $\alpha$ satisfies (51) then $\alpha^{-1}: G^{\prime} \rightarrow G$ also satisfies (51) so $\alpha$ is in fact a natural isomorphism of duality-preserving functors.

We noted in Lemma 2.29 that an exact duality-preserving functor induces a homomorphism of Witt groups. The following lemma says that naturally isomorphic duality-preserving functors induce the same homomorphism on Witt groups.

Lemma A.2. Suppose $(G, \Psi, \eta),\left(G^{\prime}, \Psi^{\prime}, \eta\right): \mathcal{C} \rightarrow \mathcal{D}$ are duality-preserving functors which respect exact sequences and $\alpha:(G, \Psi, \eta) \rightarrow\left(G^{\prime}, \Psi^{\prime}, \eta\right)$ is a natural isomorphism. If $\left(V, \phi: V \rightarrow V^{*}\right)$ is a hermitian form in $\mathcal{C}$ then there is a natural isomorphism between the induced hermitian forms $(G(V), \Psi G(\phi)) \cong\left(G^{\prime}(V), \Psi^{\prime} G^{\prime}(\phi)\right)$. Let $\zeta=1$ or -1 . The duality-preserving functors $(G, \Psi, \eta)$ and $\left(G^{\prime}, \Psi^{\prime}, \eta\right)$ induce the same homomorphism of Witt groups $W^{\zeta}(\mathcal{C}) \rightarrow W^{\zeta \eta}(\mathcal{D})$.

Proof. In the diagram

$$
\begin{array}{cr}
G(V) \stackrel{G(\phi)}{\longrightarrow} G\left(V^{*}\right) \stackrel{\Psi_{V}}{\longrightarrow} G(V)^{*} \\
\alpha_{V} \downarrow & \alpha_{V^{*} \downarrow} \\
G^{\prime}(V) \underset{G^{\prime}(\phi)}{\longrightarrow} G^{\prime}\left(V^{*}\right) \underset{\Psi_{V}^{\prime}}{\longrightarrow} G^{\prime}(V)^{*}
\end{array}
$$

the left-hand square commutes by the naturality of $\alpha$ while the right-hand square commutes because $\alpha$ satisfies equation (51). The Lemma follows easily.

It is a consequence of Lemma A.2 that an equivalence of hermitian categories induces an isomorphism of Witt groups.

\section{A.1 The covering construction}

In this section we prove that the covering construction $B$ respects changes to coefficients (Proposition 5.7). We need one more observation which is straightforward to verify:

Lemma A.3. If $A \rightarrow A^{\prime} \rightarrow A^{\prime \prime}$ are ring homomorphisms then the diagram

$$
\begin{aligned}
& A^{\prime \prime} \otimes_{A} V^{*} \longrightarrow A^{\prime \prime} \otimes_{A^{\prime}}\left(A^{\prime} \otimes_{A} V^{*}\right) \\
& \stackrel{\downarrow}{\perp} A^{\prime \prime} \otimes_{A^{\prime}}\left(A^{\prime} \otimes_{A} V\right)^{*} \\
& \left(A^{\prime \prime} \otimes_{A} V\right)^{*} \leftarrow\left(A^{\prime \prime} \otimes_{A^{\prime}}\left(A^{\prime} \otimes_{A} V\right)\right)^{*}
\end{aligned}
$$

of natural isomorphisms is commutative. 
Proof of Proposition 5.7. Suppose $A \rightarrow A^{\prime}$ is a ring homomorphism and $V$ is a module in $\mathcal{S}$ ei $(A)$. The natural isomorphism

$$
\left(A^{\prime} \otimes_{A} V\right)\left[F_{\mu}\right] \rightarrow A^{\prime}\left[F_{\mu}\right] \otimes_{A\left[F_{\mu}\right]}\left(V\left[F_{\mu}\right]\right)
$$

induces a natural isomorphism (see Lemma 2.9)

$$
\left\{\alpha_{V}\right\}_{V \in \mathcal{S e i}(A)}: B\left(A^{\prime} \otimes V\right) \rightarrow A^{\prime}\left[F_{\mu}\right] \otimes_{A\left[F_{\mu}\right]} B(V) .
$$

We aim to show that $\alpha$ is a natural isomorphism between duality-preserving functors

$$
(B, \Phi,-1) \circ\left(A^{\prime} \otimes_{A}, \Pi, 1\right) \rightarrow\left(A^{\prime}\left[F_{\mu}\right] \otimes_{A\left[F_{\mu}\right]}, \Upsilon, 1\right) \circ(B, \Phi,-1) .
$$

Applying Lemma A.3 to both composites in the commutative square

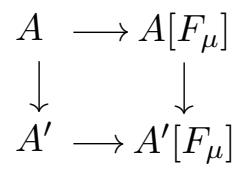

of ring homomorphisms one obtains commutative diagrams

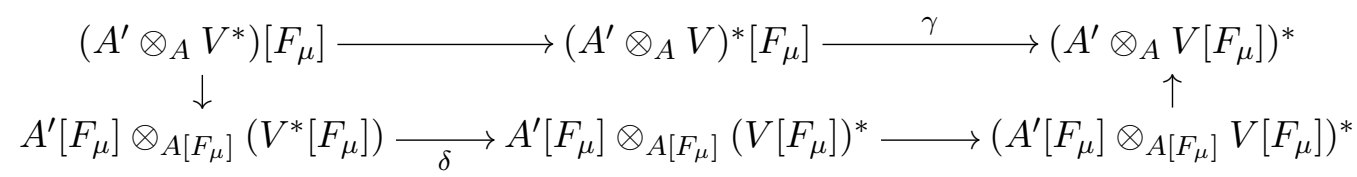

where $\gamma= \pm \Pi_{A^{\prime} \otimes V}\left(1-z^{ \pm}\right)$and $\delta=1 \otimes \pm \Pi_{V}\left(1-z^{ \pm}\right)$and hence the commutative diagram

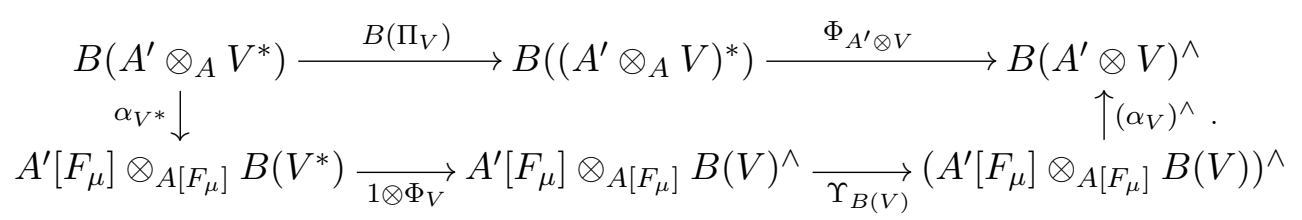

Thus $\alpha$ is a natural transformation between duality-preserving functors as claimed. It follows by Lemma A.2 that the diagram (36) of Witt group homomorphisms commutes. The proof of Proposition 5.7 is complete. 


\section{A.2 Hermitian Morita Equivalence}

In this section we prove that hermitian Morita equivalence respects dualitypreserving functors (Proposition 3.22). Let $\left(G, \Psi, \eta^{\prime}\right): \mathcal{C} \rightarrow \mathcal{D}$ denote a duality-preserving functor and let $M \in \mathcal{C}, E=\operatorname{End}_{\mathcal{C}} M$ and $E^{\prime}=$ $\operatorname{End}_{\mathcal{D}} G(M)$.

We shall define a natural isomorphism between the composite functors

$$
\begin{aligned}
\alpha:\left(E^{\prime} \otimes_{E}, \Pi, 1\right) \circ & \left(\operatorname{Hom}(M, \ldots), \Omega^{b}, \eta\right) \\
& \stackrel{\simeq}{\longrightarrow}\left(\operatorname{Hom}(G(M), \ldots), \Omega^{\eta^{\prime} \Psi_{M} G(b)}, \eta \eta^{\prime}\right) \circ\left(G, \Psi, \eta^{\prime}\right)
\end{aligned}
$$

If $\left.N \in \mathcal{C}\right|_{M}$ then $\operatorname{Hom}(M, N)$ is a left $E$-module for the action

$$
f . \theta=\theta \bar{f}=\theta b^{-1} f^{*} b
$$

where $f \in E$ and $\theta \in \operatorname{Hom}(M, N)$. The group $\operatorname{Hom}(G(M), G(N))$ is regarded as a left $E^{\prime}$-module in the same way. Define

$$
\begin{aligned}
& \alpha_{N}: E^{\prime} \otimes_{E} \operatorname{Hom}_{\mathcal{C}}(M, N) \rightarrow \operatorname{Hom}_{\mathcal{D}}(G(M), G(N)) \\
& \quad f \otimes \gamma \mapsto f . G(\gamma)=G(\gamma) \bar{f}=G(\gamma)\left(\eta^{\prime} \Psi_{M} G(b)\right)^{-1} f^{*}\left(\eta^{\prime} \Psi_{M} G(b)\right) .
\end{aligned}
$$

Since $\alpha_{N}$ is an isomorphism in the case $N=M$ it follows that $\alpha_{N}$ is an isomorphism for all $\left.N \in \mathcal{C}\right|_{M}$. It is easy to see that $\{\alpha\}_{\left.N \in \mathcal{C}\right|_{M}}$ is a natural transformation

$$
\left(E^{\prime} \otimes_{E}{ }_{-}\right) \circ \operatorname{Hom}\left(M,_{-}\right) \rightarrow \operatorname{Hom}\left(G(M),{ }_{-}\right) \circ G .
$$

One must check that $\alpha$ is a natural transformation of duality-preserving functors. By equations (19) and (51) one must show that

$$
\alpha_{N}^{*} \Omega_{G(N)}^{\eta^{\prime} \Psi_{M} G(b)} \operatorname{Hom}\left(G(M), \Psi_{N}\right) \alpha_{N^{*}}=\Pi_{\operatorname{Hom}(M, N)}\left(1 \otimes \Omega_{N}^{b}\right)
$$

This equation can be checked by direct calculation, substituting the formulae (52), (28) and (20) for $\alpha, \Omega$ and $\Pi$ respectively and applying the naturality of $\Phi$ and the equation (18). This completes the proof of Proposition 3.22 .

\section{References}

[1] A. A. Albert. Structure of Algebras, volume 24 of American Mathematical Society Colloquium Publications. American Mathematical Society, New York, 1939. 
[2] P. Ara. Finitely presented modules over Leavitt algebras. Journal of Pure and Applied Algebra, 191:1-21, 2004.

[3] R. C. Blanchfield. Intersection theory of manifolds with operators with applications to knot theory. Annals of Mathematics (2), 65:340-356, 1957.

[4] F. Borceux. Handbook of Categorical Algebra 1. Basic Category Theory, volume 50 of Encyclopedia of Mathematics. Cambridge University Press, 1994.

[5] S. E. Cappell and J. L. Shaneson. The codimension two placement problem, and homology equivalent manifolds. Annals of Mathematics (2), 99:277-348, March 1974.

[6]_ and Link cobordism. Commentarii Mathematici Helvetici, 55:20-49, 1980.

[7] T. D. Cochran and K. E. Orr. Homology boundary links and Blanchfield forms: Concordance classification and new tangle-theoretic constructions. Topology, 33(3):397-427, 1994.

[8] P. M. Cohn. Free ideal rings. Journal of Algebra, 1:47-69, 1964.

[9] Free Rings and their Relations. London Mathematical Society Monographs, 2. Academic Press, London, 1971.

[10] _ Free Rings and their Relations. London Mathematical Society Monographs, 19. Academic Press, London, 2nd edition, 1985.

[11] _ Localization in general rings, a historical survey. Pages 5-23 in this volume.

[12] and W. Dicks. Localization in semifirs. II. J.London Math.Soc. (2), 13(3):411-418, 1976.

[13] W. Dicks and E. Sontag. Sylvester domains. J. Pure Appl. Algebra, 13(3):243-275, 1978.

[14] J. Duval. Forme de Blanchfield et cobordisme d'entrelacs bords. Commentarii Mathematici Helvetici, 61(4):617-635, 1986.

[15] M. Farber. The classification of simple knots. Uspekhi Mat. Nauk, 38(5):59-106, 1983. Russian Math. Surveys 38:5 (1983) 63-117. 
[16] _ Hermitian forms on link modules. Commentarii Mathematici Helvetici, 66(2):189-236, 1991.

[17] Noncommutative rational functions and boundary links. Mathematische Annalen, 293(3):543-568, 1992.

[18] _ and P. Vogel. The Cohn localization of the free group ring. Mathematical Proceedings of the Cambridge Philosophical Society, 111(3):433-443, 1992.

[19] R. H. Fox. Free Differential Calculus. I: Derivation in the Free Group Ring. Annals of Mathematics (2), 57(3):547-560, 1953.

[20] P. Gabriel. Des catégories abéliennes. Bulletin de la Société Mathématique de France, 90:323-448, 1962.

[21] _ and M. Zisman. Calculus of Fractions and Homotopy Theory, volume 35 of Ergebnisse der Mathematik und ihrer Grenzgebiete. Springer, New-York, 1967.

[22] C. Kearton. Blanchfield duality and simple knots. Transactions of the American Mathematical Society, 202:141-160, 1975.

[23] Cobordism of knots and Blanchfield duality. Journal of the London Mathematical Society (2), 10(4):406-408, 1975.

[24] M. A. Kervaire. Les noeuds de dimensions supérieures. Bulletin de la Société Mathématique de France, 93:225-271, 1965.

[25] Knot cobordism in codimension two. In Manifolds-Amsterdam 1970, Lecture Notes in Math., 197, Springer, Berlin, 1971, pp. 83-105.

[26] M.-A. Knus. Quadratic and Hermitian Forms over Rings. Grundlehren der Mathematischen Wissenschaften, 294. Springer, Berlin, 1991.

[27] K. H. Ko. Seifert matrices and boundary link cobordisms. Transactions of the American Mathematical Society, 299(2):657-681, 1987.

[28] _ A Seifert-matrix interpretation of Cappell and Shaneson's approach to link cobordisms. Mathematical Proceedings of the Cambridge Philosophical Society, 106:531-545, 1989.

[29] T. Y. Lam. A First Course in Noncommutative Rings. Springer, New York, 1991. 
[30] S. Lang. Algebra. Addison-Wesley, 3rd edition, 1993.

[31] J. Levine. Invariants of knot cobordism. Inventiones Mathematicae, 8:98-110, 1969. Addendum, 8:355.

[32] Knot cobordism groups in codimension two. Commentarii Mathematici Helvetici, 44:229-244, 1969.

[33] Knot modules I. Transactions of the American Mathematical Society, 229:1-50, 1977.

[34] J. Lewin. Free modules over free algebras and free group algebras: The Schreier technique. Transactions of the American Mathematical Society, 145:455-465, November 1969.

[35] S. Mac Lane. Categories for the Working Mathematician. Number 5 in Graduate Texts in Mathematics. Springer, 1971.

[36] Homology. Springer, 1995. Reprint of the 1975 edition.

[37] J. W. Milnor. On isometries of inner product spaces. Inventiones Mathematicae, 8:83-97, 1969.

[38] W. Mio. On boundary-link cobordism. Mathematical Proceedings of the Cambridge Philosophical Society, 101:259-266, 1987.

[39] W. Pardon. Local surgery and applications to the theory of quadratic forms. Bulletin of the American Mathematical Society, 82(1):131-133, 1976.

[40] _ Local surgery and the exact sequence of a localization for Wall groups. Memoirs of the American Mathematical Society, 12(196):iv+171, 1977.

[41] H.-G. Quebbemann, W. Scharlau, and M. Schulte. Quadratic and Hermitian forms in additive and abelian categories. Journal of Algebra, 59(2):264-289, 1979.

[42] A. A. Ranicki. Exact Sequences in the Algebraic Theory of Surgery. Mathematical Notes 26. Princeton University Press, New Jersey; University of Tokyo Press, Tokyo, 1981.

[43] _ High-dimensional Knot Theory. Springer, Berlin, 1998. 
[44] Blanchfield and Seifert algebra in high dimensional knot theory. Moscow Math. J. 3:1333-1367, 2003. arXiv:math.GT/0212187.

[45] Noncommutative localization in topology. Pages 81-102 in this volume. arXiv:math.AT/0303046.

[46] _ and D. Sheiham. Blanchfield and Seifert algebra in high dimensional boundary link theory I. Algebraic $K$-theory. arXiv:math.AT/0508405.

[47] N. Sato. Free coverings and modules of boundary links. Transactions of the American Mathematical Society, 264(2):499-505, April 1981.

[48] W. Scharlau. Quadratic and Hermitian forms. Grundlehren der Mathematischen Wissenschaften, 270. Springer, Berlin, 1985.

[49] A. H. Schofield. Representations of rings over skew fields, volume 92 of London Mathematical Society Lecture Note Series. Cambridge University Press, 1985.

[50] D. Sheiham. Non-commutative characteristic polynomials and Cohn localization. Journal of the London Mathematical Society (2), 64(1):1328, 2001. arXiv:math.RA/0104158.

[51]_Invariants of Boundary Link Cobordism, volume 165 of Memoirs of the American Mathematical Society. American Mathematical Society, 2003. arXiv:math.AT/0110249.

[52] J. R. Smith. Complements of codimension-two submanifolds - III cobordism theory. Pacific Journal of Mathematics, 94(2):423-484, 1981.

[53] V. Srinivas. Algebraic K-theory, volume 90 of Progress in Mathematics. Birkhäuser, Boston, 1996.

[54] P. Vogel. Localisation in algebraic L-theory. In Proc. 1979 Siegen Topology Conf., Lecture Notes in Mathematics, 788, pages 482-495. Springer, 1980.

[55] On the obstruction group in homology surgery. Publ. Math. I.H.E.S., 55:165-206, 1982.

International University Bremen

Campus Ring 1

Bremen 28759

Germany 